University of Louisville

ThinkIR: The University of Louisville's Institutional Repository

Electronic Theses and Dissertations

1938

\title{
The loss in drying power of black paints due to aging.
}

J. E. Rose

University of Louisville

Follow this and additional works at: https://ir.library.louisville.edu/etd

Part of the Chemical Engineering Commons

\section{Recommended Citation}

Rose, J. E., "The loss in drying power of black paints due to aging." (1938). Electronic Theses and Dissertations. Paper 1881.

https://doi.org/10.18297/etd/1881

This Master's Thesis is brought to you for free and open access by ThinkIR: The University of Louisville's Institutional Repository. It has been accepted for inclusion in Electronic Theses and Dissertations by an authorized administrator of ThinkIR: The University of Louisville's Institutional Repository. This title appears here courtesy of the author, who has retained all other copyrights. For more information, please contact thinkir@louisville.edu. 


\section{$+\infty$ \\ UNIVIRSITY OF IOUISVIIIA}

The Loss in Drying Power of Biack Paints Due to Afine

A Dissertation

Subaitted to the Faculty

of the Graduate School

In partial Fulfillment of the

Requirement for the Deeree of

Master of Chomical bucine erine

Department of Chemical incineerine

By

J. B. Rose

2938 
The Loss in Drying Power of Black Paints Due to Aging

Director: Dr. K. C. Ernst.

Approved by keading Committee:

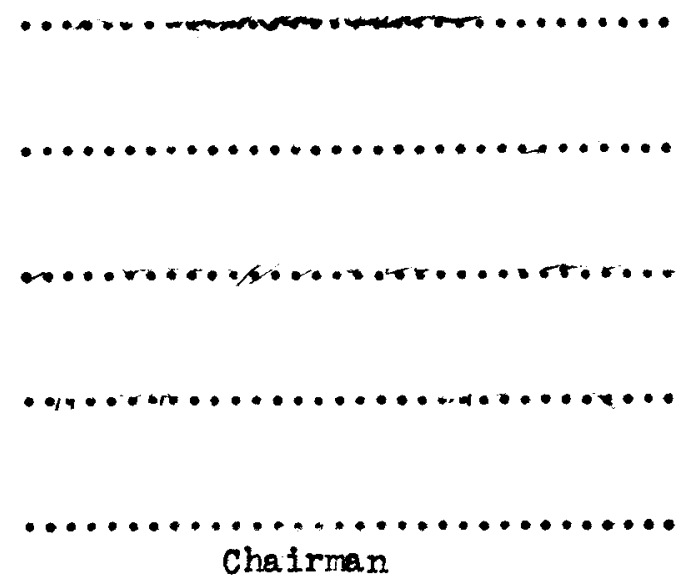


T BLE OF UONIENTS

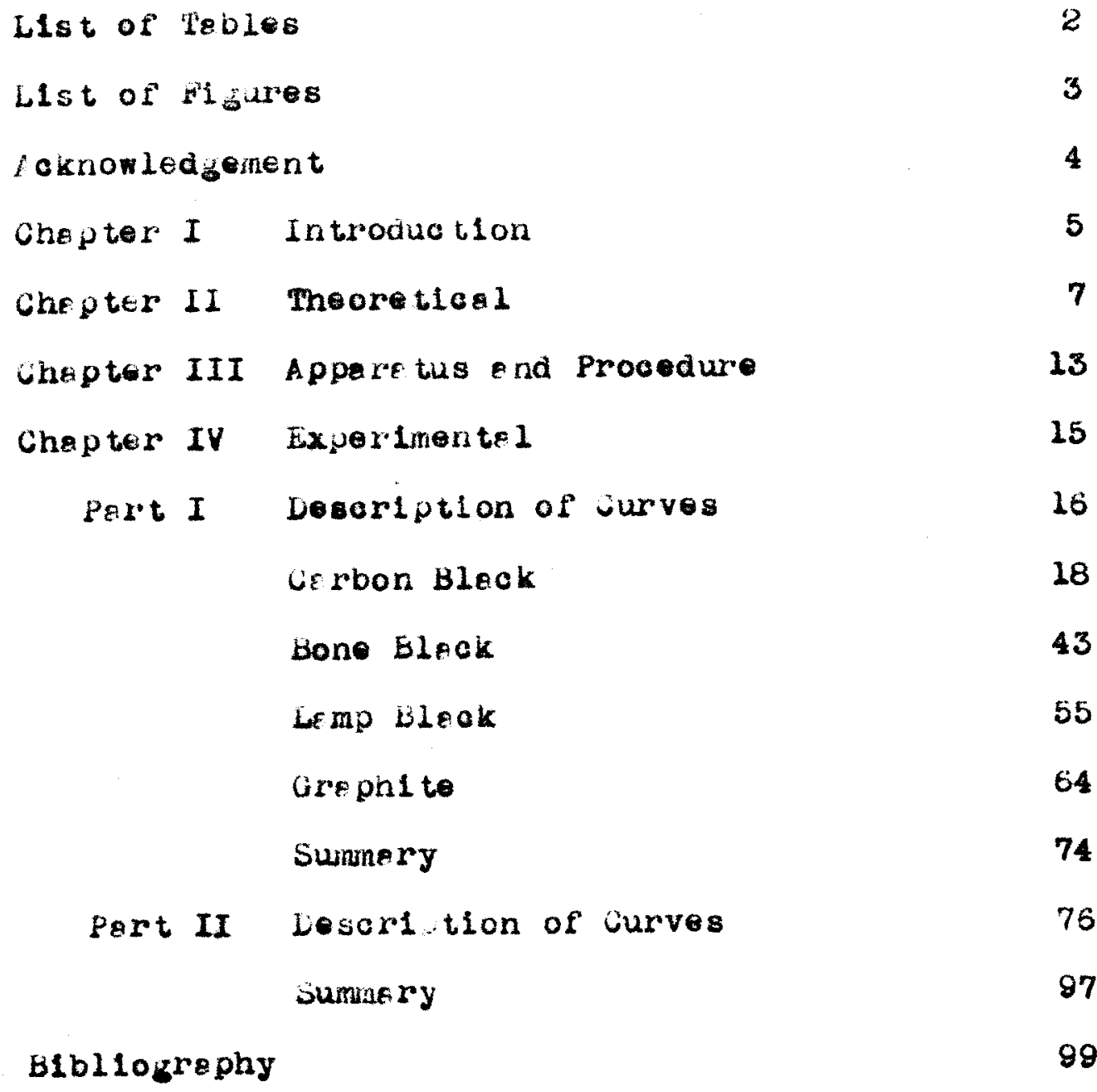


LIST OF TABLES

Teble No.

Page

1 List of Linseted 011 - Lrier - P1gment Wla tures Studied

22

2 Drying Le te for Linseed uli contelning 1 Grem Leed end 3.5 Grams Cerbon Bleck per 100 Groms 01123

Drying Dete for Linseed 011 Conteining 1.5 Grems Lead end 3.5 Grems Cerbon Black per 100 Grama 011

25

4

Drying De te for Linseed oll Conteinlng 2 Grems Lefd end 3.5 Grams ur roon Bleck per 100 Grams 011

Drying Le te for Linseed O1l Conteining 1 Grem Leed end 7 Grems cerbon Black per 100 Grams of 011

29

6

Drylny Dete for Hinseed 011 Conteining 1.5 Grems Leed end $i$ Grems Cerbon Bleck per 100 Grems 011

Drying De te for Linseed oll Conteining 2 Grams Leed end 7 Grems Cerbon Bleck per 100 Grems 011

Drying De te for Linseed 011 Uonteinint 0.025 Grems Cobelt end 3.5 Urems carbon Bleck per 100 Grems oll

Drylrg Dete for Linseed OLl Contelning 0.025 Grams Cobelt end 7 Grems cerbon Bleck per 100 Grams 011

10 Drying vete for Linseta oil conteining 0.04 Grems Cobe 1 and 3.5 Grems urroon Bleck per 100 Groms 011

11 Drying Deto for ulnseed 011 conteining 0.04 Grem Cobelt and 7 Grems verbon Bleck per 100 Grems 011

Drylng Lete for Linseed 011 Conteining 1 Grom Lead and 10 urems Bonebleak per 100 Grams 011

13 Drylag De te for Linseed O11 vonteining 2 Grems Leed end 10 Grems bone black per 100 Grms 011

14 Drying Deto for Linseod 011 Contelning 1 Grem Leed end 20 Grems bone black per 100 Grems 011

15 Drylne Dete for Linseed 011 Con tolning 2 Grems Loed find 20 Grems Bone bleck per 100 Grems 011 
Toblo lo.

Pege

16 Drylng De te for Linseed oll conteining 1 Gram Lead end 7 Grems Lemp blaok per 100 Grams 011

56

17

Drylng Dete for Linseed 011 conteining 2 Grems Loed and 7 Grams Lamp bleck per 100 Grems 011

Drying Date for Linseed 011 Contelning 1 Gram Leed and 14 Grams Lamp bleck per 100 Grems 011

19

Drying Date for tinseed oll Conteining 2 Grems Lead end 14 Grems LFmp bleck per 100 Grems 011

20

Drying De te for Linee 011 Conteining 1 Grem Lead end 14 Grems Grephl to per 100 Grems 011

21 Dryine Date for Linseed 011 Contelning 2 Orems Lead and 14 Grems Grephito per 100 Grams 011

Drying Dete for ulnsegd oll conteining $1 \mathrm{Grem}$ Lead and 28 Grems Grephl te per 100 Grams 011

Drying Date for Linseed oll Contelning 2 Grams Lead and 28 Grems Grephlte per 100 Grems 011

Drying De ta far LInseod OLl Contalning 0.1 Grem Aluminum Steerate, 0.04 Gran Cobe1t, sud 3.5 Grems Cerbon bleck per íoo Grems 011

Drying Dete for Linseed oll Contelning 0.1 Grems 21ne, 0.04 Grem viobelt, nd 3.5 Grems Cerbon Bleck per 100 Grems 011

Drying Le te for Linseed 011 contalning 0.1 Gram ZIne, I Grem Leed, and 3.5 Grems Cerbon Bleck per 100 Grems 011

Drylag Le te for Linseed 011 contelning 0.04 Grems Cobs It and 3.5 Grems Cerbon Bleck (treeted ith ammoniacel cuprous chloride) per 100 Grams 011

Dryins De te for Linseed 011 contelning 0.04 Grems Cobelt and 3.5 Grems cerbon Bleck (treeted with $25 \%$ potessium hydroxide) per 100 Grems 011 
Teble No.

30 Drylug Lete for Linseed 011 Conteining 0.25 Urm Lead, is Lithe rue, 0.04 Grem Cobelt, fnd 3.5 Grems Cerbon Bleck per 100 Grems 011

31 Dryine Dete for winsed oll Conteining 0.5 Grams Leed, s Li therge, 0.04 Gram Cobalt, and 3.5 Grems cerbon Bleok per 100 arems 011 
LIST OF PIGURES

Figure Nio.

Pege

1 Explenstion of Induction Perlod

17

2

Drying to to for Linseed oil Conteining 1 Grem Lead and 3.5 Grems carbon b.eok per $100 \mathrm{Grems} 011$

Drylng ke te for Linsed 011 contelning 1.5 Grems

Lead a 3.5 Grems Carbon Black per 100 Grems 011

Drying ke te for Linseed oll Contelning 2 Grems Lesd end 3.5 Grams Cerbon Blook per 100 Grems 011

Drying to te for inseed ofl Conteining 1 Gram Leed end 7 Grems cerbon Bleck per $100 \mathrm{Grems}$ of 011

Drylng kate for Linseed oil Conteining 1.5 Grams Leed nd 7 Grams Cerbon Bleok per 100 Grems 011

Drying he to for Linseed 011 Contalnine 2 Groms Lead end 7 Grems Corbon Bleck per 100 Grams 011

8 Drylng tete for Linsed 011 Conteining 0.025 Grems vobelt end 3.5 Grems Cerbon Black per 100 arems 01136

Drying ke te for Linseed 011 Contelning 0.025 Grems Cobplt and 7 Grems Carbon Bleck per 100 Grems 011

10 Drying hite for Linge 011 Contelnino 0.04 Grems Cobelt and 3.5 Grems Cerbon Blfok per 100 Grems 01140

11 Drying he to for inseed 011 Containing 0.04 Grom Cobelt and 7 Grems Carbon Bleck por 100 Grams 011

12 Drying he to for Linseed Oll Contelning I Orem Loed and 10 Grems Boneblock per 100 Grems o1

Drying he te for Linseed 011 Conteining 2 Grems Leed end 10 Grems Boneblack per 100 Grems oll

14 Dryine lis te fur Linseed o1I Conteinins I Grom Lead and 20 Grams Bonebleck por 100 Grems ot 
Plgure No.

Drying lie te for Linseed 011 Conteining I Grem Leed and 7 Grams Lemp bleck per 100 urams ot 1

Dry ing kete for Linseed Oll conteining 2 Grams Lesd ond 7 Grams Lamp bleck per luo Grems U11

Lrying to to for cinseed uil Containing 1 Grem Loed end 14 Groms tomp bleck per 100 Grams 011

Drying the for for inseed oll Conteinirig 2 orems Leed end 14 Grems Lemp bleck per 100 Grems U1l

Drying the to for Linseed OLI Conteining 1 Grom Lead and 14 Grems Graphlte per 100 Grems oll

21 Drying ke te for Lingeed 011 Lonteining 2 Grems Loed a nd 14 Grems Grephlte per $100 \mathrm{Gramis} 011$

Drying he te for ilrseed Oll Conteining 1 Grem Lead a nd 28 Grams Grephite per 100 Grems 011

Drying he to for Linseed U1l conteining 0.1 Grem Aluminum Steere te, 0.04 Grem Cobsit, and 3.5 Grams Cerbon Black per 100 areme U1l

Drying the te for Lineed oil Contalning 0.1 Grems Lino, 0.04 Grem Cobelt, end 3.5 Grsms Cerbon Bleck per 100 Grems 011

Drying te te for Linseed 011 Contelning $0.1 \mathrm{Gram}$ Zine, $1 \mathrm{Grem}$ Lerd, end $3.5 \mathrm{Grems}$ Carbon Bleck per 100 Órems 011

Drying the for Linseed 011 Conteining 0.04 Groms Cobelt ond 3.5 Grams Cerbon Black (tree ted with ammoniecel cuprous chloride) per 100 Grems 011

Dry In ke to for Linseed Oll conte Ining 0.04 Grems Cobe 1 t and 3.5 Grema Carbon black (tree ted ith $25 \%$ potessi um hydroxide) per 100 Grams 011 
Figure No.

30

Drylng the for Lingeed 011 Contelnine 0.25 Grem Leed, 8 Li therge, 0.04 Gram Cobe $1 t$, ond 3.5 Grems Gerbon Bleck per 100 Grams 011

31

Drying tif te for Linseed oll Containing 0.5 Grems Lead, $s$ ti tharge, 0.04 Grem Cobelt, end 3.5 Grams cerbon bleck per 100 Grams 011 


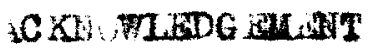

The euthor wishes to acknowledge his appreciation for the ald given by Dr. R. C. Brnst. who direoted his research. 
CHAPTBR I

IHTRODUCIIUF 
The dryinc time of many black painte increases while the paints are stored in containers prior to salo. This investigation is a study of the 1088 in dryine time c.use by aeing.

This work was concerned th the preparation of dryine rate curves for suples of linseed oil having carbon black. lamp black, bone black, and graphite as pigent. and having lede and cobalt naphthenates as driers.

The drying $r$ the curves corer b period of fourteen munths und iriclude tie data of Bennett (1) for the first tro monthe.

In ad aition to the preparation of drying rate curves, methods for oorrecting the loss in dryine power, were studied in a comparable manner.

Carbon black, the pigment which showed the greateat $20 s 8$ in dryine ability, was used exclusively in the latter part of the investigation. 
CHAPTIR II

THEORY 
IInseed oil is a Viariable combination of lyceryl esters of saturisted and unsaturated fatty aode and contains approximately $93 \%$ tatty acids and $7 \%$ elyoerol in combination. The unsaturated fat ty acide are ohlefly oleio, Inoleic, and linolenic. Small amounts of the saturated stearic acld are also present. 0lelo acid. containine one double bond and resesented by the formula $c_{17}{ }^{\mathrm{H}} 3^{\mathrm{COOH}}$. loes not impart erying qualities to the oil. but is capable of beinc oxidized at hicher temperatures. The unaturated fut ty acids that cause the oil to dry are inoleic. containing two ouble bonds and havict the formula $\mathrm{C}_{2}{ }^{\mathrm{H}} 3 \mathrm{H}^{\mathrm{COOH}}$; and linolenio, containing three Louble bonde and having the formula $\mathrm{C}_{1} 7_{29} \mathrm{COOH}$.

Iinseed oll undercoes oxidation and polywerization durine the dryire period. The oil is cipable tileoreticuly of absorbing $18 \%$ axyen, but actuilly the increase in we ight is unly $10-14 \%$ because of the $208 \mathrm{~s}$ of the rolatile production of decomposition. The oil oxidizes, thereby ading oxyeen to the double bonds.

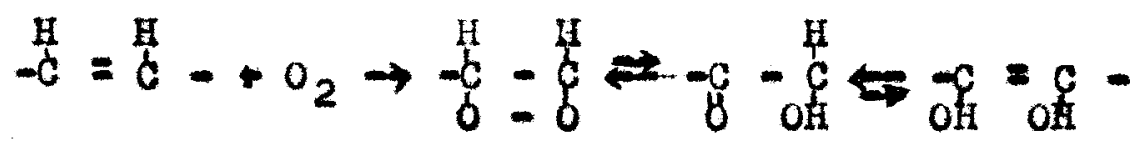

The absarption of oxycen inoreases the nolecular (4) weight and polarity to a point where molecular eruwtis are formel. At a certain stage tile se frowtho became insoluble 
in the liquid phose of oil forming mall nuoeli collo1ds. As the eroups of noleoules separate. they adsorb the remaining liquid, thereby foming a solid. The final solid is oalled linoxyn, and on actne is continuouely broken down by further oddation into fatty acide of Bmazler moleoular weighto and into volatile products. ouch as curbon lioxide.

The natural aryite time of 1 inseed oil is three to four days. This time can be reduced to several hours by the addition of metalif ostalysto called iriers. The three metals comonly used as driers are lead, cobalt, and mancanese. It is found that loal acto as a coaculator of the film, exhibiting little of the property know as top-drying. Cobalt and mane sne do not show this proverty of coagulating the film, but act as pwerful surface iriers. The addition of I mpblack and carbon black to linseed oil vory distinctly inhibits the oxidation of the oil. It had cenerally been thought that this inhibition was the result of the presenoe of oily or oil soluble impurities in the pigments. Rholes and Goldamith (6) showed, howerer, that this was not the oause, and stated that the offeot of the aarbon pigmente in dezayine the oxidation of raw Iinseed all was due to cunti ruous adsorption of the intermediate oxidation product wioh acts as the true catalyst in the drying reaction. They al so showed that carbon 
pligments only $311 \mathrm{~g}$ tly retrid the oxldetion of freshly prepered peints contining cobelt drier, the inhiblting efiect becoming more pronounced os the peints were allowed to stend. This, they steted, wes due to the greduel edsorption of the coblit drier by the plgment.

Gas cerbon blacks adsorb ler que qutitles of air on the surfece of the pfrticles, contelining cerbon monoxide, oxygen, and ofrbon dloxlde. It is posslole the these geses af ect the drying of the oll when mede into $\varepsilon$ point. when the right amount of drier wes gdded for correct drying tine of fresily prepered point, the drying perlod would be too long efter the semple hed eged. when the correct emount of arier wes edied for the proper drylng rete $\varepsilon$ t the end of the jer $x$, film of the freshly prepered peint would become brittle and not durpble.

It would seem, therefore, thet the rete of edsorption of drler from the oll vehicle is rether slow, end the bleck becones setureted only ifter e long perlod.

In order to determine the seturetion quentity of drier, E series of tests was run to determine the edsorption of drier by cerbon bleck from $E$ nepthe solution of the drier. This series of tests wes performed ny the Blnney ond Smith Compeny (2). Dete were obteined with verlous cerbon blacks, uslag Soligen driers of lead, cobelt, end mengenese in a nepthe solution. Actuel pelnt grinds would heve to be mede to esteblish definitely the proper emount of drier 
and proper method for overcoming this aifficulty.

f solution to this problem would be to develop method oy anich this drier adsorption could be mede in short time. This might be done by treeting the plgment with solution of the required emount of arier previous to grinding, or by edding the proper emount arler to the peint for cdsorption before grinding snd then the subsequent eddition of drier for drying purposes.

Investigetions by the ontreal Club (5), on edsorption of arler by inorgenic ploments, show by photomicrogrephs thet there is a soep farmetion whlch keeps the plgment pertioles sepereted, preventing 888lomeretion or flocculetion wh the oxide. Il $\mathrm{J}_{2}$ snd fe, $\mathrm{O}_{3}$ not heving 8 sopp leyer on the plgnect surfoce to prevent rlocouletion formed agglomeretes. This flocculetion epperred to teke pleas within \& fow weeks efter srinding during which time the metell10 soep edded es drier wes edsorbed to the surfeco of the plgment. Some of this drier was adsorbed during the time thet flocoulftion wos teking plece, fad becme entrepped Ithin the egolomeretes thereby being removed from sctivity. They showed thet the lost drying power might be restored if the whole of the adsorbed drier could be libere ted from the pignent egglomeretes by regrinding. Jones 8 nd fett $(3)$, dealing with loss of drying strength Wh cerbon bleck peints, stete thet cerbon bleck is very susceptible to flooouletion. When the plgment is ground 
in vehicle cooked with litherge, flocouletion is mintmized end the arying time of the peint ls stebilized. the edaltion of litherge to the vehicle prior to cooking eruses the formetion of leed soeps which during grinding tend to coet the olgment pertleles and thereby prevent flocculetion end edsorption of edded arlers. 
CHA PWEK III

\section{APPARATUS ND PROCELUKE}


The epperetus consisted of choln-ometlc belenoe end glass pletes $9 \times 12$ upon which films of peint .002 - .0025 inch thick were spreed by "arewing" procese.

Two metel guege strips (.002 inch thlck) were pleoed on the auter edges of eech plate; pelnt was spread thickly on the plete with smell brush, and metel blede arewn ecross the plete in cortact with the gugge strips. Thus, there was a cleerence of .002 inch between the surfece of the gless plete end the blade. These films of pelnt relghed betreen .15-.25 grems.

The stmples were mixtures of 11 nseod 011 , pigments, end ariers. Cerbon bleck, lempbleok, bonobleck, end grephite were the pigments, frd cobalt end leed Huodex were the drlers. witherge, potess Ium hydroxide, Cuprous chlorlde, and the Nuodex were used durlng the treetment of cerbon ble ck in certain mixtures.

The films were arled inder constant temperature conditions $\left(25=l^{\circ} \mathrm{C}\right)$ in the presenoe of $11 \mathrm{ght}$ from a dalight type bulb. 
CH PTER IV

$$
\text { PALTI }
$$

EXPERIUENTLL 
The semples investiof ted, being mixtures of 1 inseed 011 wth e1ther orbor black, lampbleok, grephite, or boroblack, wth olther leed or cobelt Nuodex as driers nere prepered in the following marner. The plgment and oll were welghed end the Nuodex drier, which in solution of e metel nephthene te in nephthe, we added with a grodueted plpette. Samples of approximetely ono-helf pint were prepered nà ground in smell pebble mill.

The compositi ons of the varlous $\mathrm{mix}$ tures studied Are glven in Table I.

The gein in meight nethod was chosen es the simplest end most diroct menner of prepering curves for comperiaons. The voletile producte of oxldetion, not teken into account, were essumed to be constent frotor ena for comperetive purposes were neglected.

The welght of oll in the film wes used s the besis for expressing the incregse in weight of the $\mathbb{1 m}$. The welght of oll in the semple res obtelned in the followlng memer: 98 grams o11, 1 grem plgment, and 1 gram drier solution geve mixture of $98 \%$ o11 content.

The increase in weight divided by the welght of 011 gevo the peroent increese from which the drying ourves were plotted.

Time (In hours) wes plotted ogeinst the pe reent inoresse In we int of oil. 
The "induction period", used extensively in the descriptions of the curves is the time when sherp $\%$ increose in weight beging. Seo Fig. I.

Figure I.

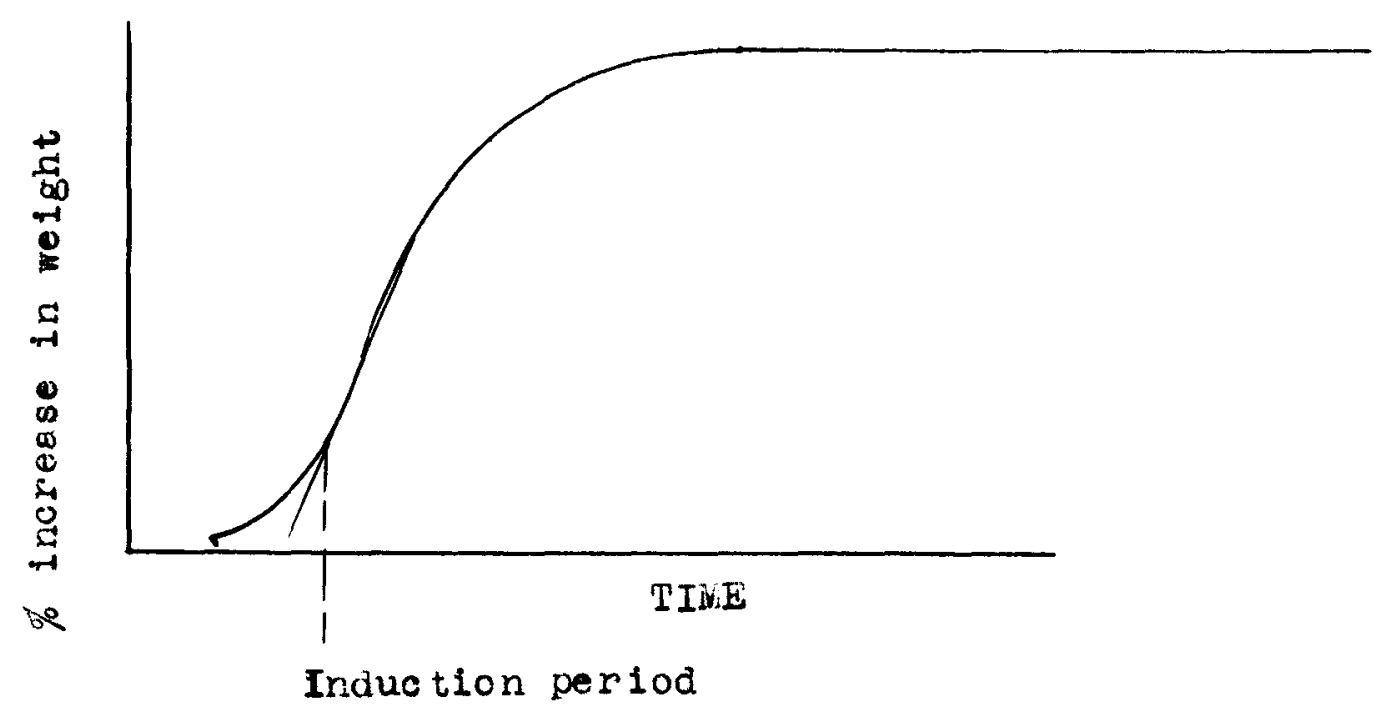


C. KBOR BLACK 
The drying curves for 1 inseed oll conteining cerbon bleck end leed drier ere shown in Figures 2 through 7. The oll mixtures thet contelned cobelt as the drier ere shown in Flgures 8 through 11.

Plgure $2(1$ grem lead and 3.5 grems cerbon bleok per 100 grams o11) showed en increse in induction period from four hours, far o freshly nede semple, to a meximum Indue tion peri od of serenteen hours, for six month's oging. The ninth and fourteenth month' curves receded to en Induction period of tourteen hours.

The induction perlod in plgure $3(1.5$ grems legd and 3.5 grems cerbon bleok por 100 grems 011) for the fresh semple ses three hours while the perlod for the sixth month's semple wos elght hours. The ninth month's induction period wes nine hars with the fourteenth month ot ten hours.

The composition of the samples of flgure 4 wes 2 grame loed and 3.5 grams cerbon bleak per 100 grams 011 . The maximum Increese in Induction perlod wes from two and one-hel f hours (frean semple) to eight hour for the fourteenth month's semple.

The greater the drier conoentretion for oertein percentege plgment the 1 ess was the loas of drying eblilty on eging the mixtures. 
The compositions of semples of 1 igures 5,6 , end 7 are respeotlvely $1-1.5-2$ grems lead end 7 grems cerbon bleok. The curves of ploures 5,6 , end 7 were compreble to those in Flores 2,3, and 4 except thet orch ehowed a loss in drying power much greeter then the oorresponding curves. Inls was probebly the result of f-ster edeorption of arier by a greater percentyge of pigmet.

The Induotion period shown in rigure 8 (.025 grama cobslt end 3.5 grems eerbon bleck per 100 grems o11) Incressed very grectly from to ur hours ( $\mathrm{resh}$ sample) to the meximum of fifty hours (six month's). The ninth end far teenth nonth per lods receded to forty and twenty-five hours respectively.

The mix tupe of Flgure 91.025 grems cobelt and 7 grems af lion bleck per 100 grams o1l) heving nore cerbon bleck, displeyed greeter loss in arying power than the semple of Flgure 8. The lreresse in induetion perlod we from elgnt hours (fresh smple) to epproximetely seventy-five hours, the mexlrium $(s 1 x$ month's eging). The ninth end fouteenth month' semples receded, as in the previous cese, to induction periods of fifty-five and forty-flve hours respectlgely.

The content of semples in 1 gure $101 \mathrm{l} .04$ grams cobalt and 3.5 grams carbon black per 100 grems oll. This mixture, conteining more arler then that of figure 8 geve. 
ohorter induction period of the freshly mode smple by two end one-he if to four hours. However, the Induotion perlods for the six month's sample were epproxime tely the ano for both floures 8 and 10 ; thus it wes shown thet this le rjer, ercentege of drier we removed from otitity during this eging period. The ninth month's ample hed en Induction period of sixty hours while the fourteenth month's semple hed an Induction period of twenty-two hours. As in rlgures 3 and 4 , gremter emount of plgment exhiblted grefter indiction perlod. The freah ample he a en Induc tion period of four hours while the $s 1 x$ month'semplo hed en induction period of epproximetely soventy-five hours, much greeter then thet sown in Pigure 10. Tho ninth frid fourteonth month's ormplos, as in Flyures 8 ond 9 dropped back to $81 x t y-f 1$ vo and forty-fivo hours reapeotively.

The cerbon bleck eamplessbewed distinct loss in ary ing power. Aging in 11 er ses resulted in 108 of drylng obl11 ty. However, many of the ninth end fourteenth month's ourres aropped behind the maximum Induction pe riod mich wes the sixth month's ourve in most oeses. Tho greater percentege of plgment showed greater los in drylog power, end greter percentages of arier exhibitod distinctly sm llor losses in arjing time oxoept in the ceses of the cobsit driers of Figures 9 and 11. 
Teble 1

Composition* of Linseed 011, Drier, end Plgment Mixtures

Sample

Number
Drier
Plgmont
Results hesults hoported in Shown in Table No. Flgure No.

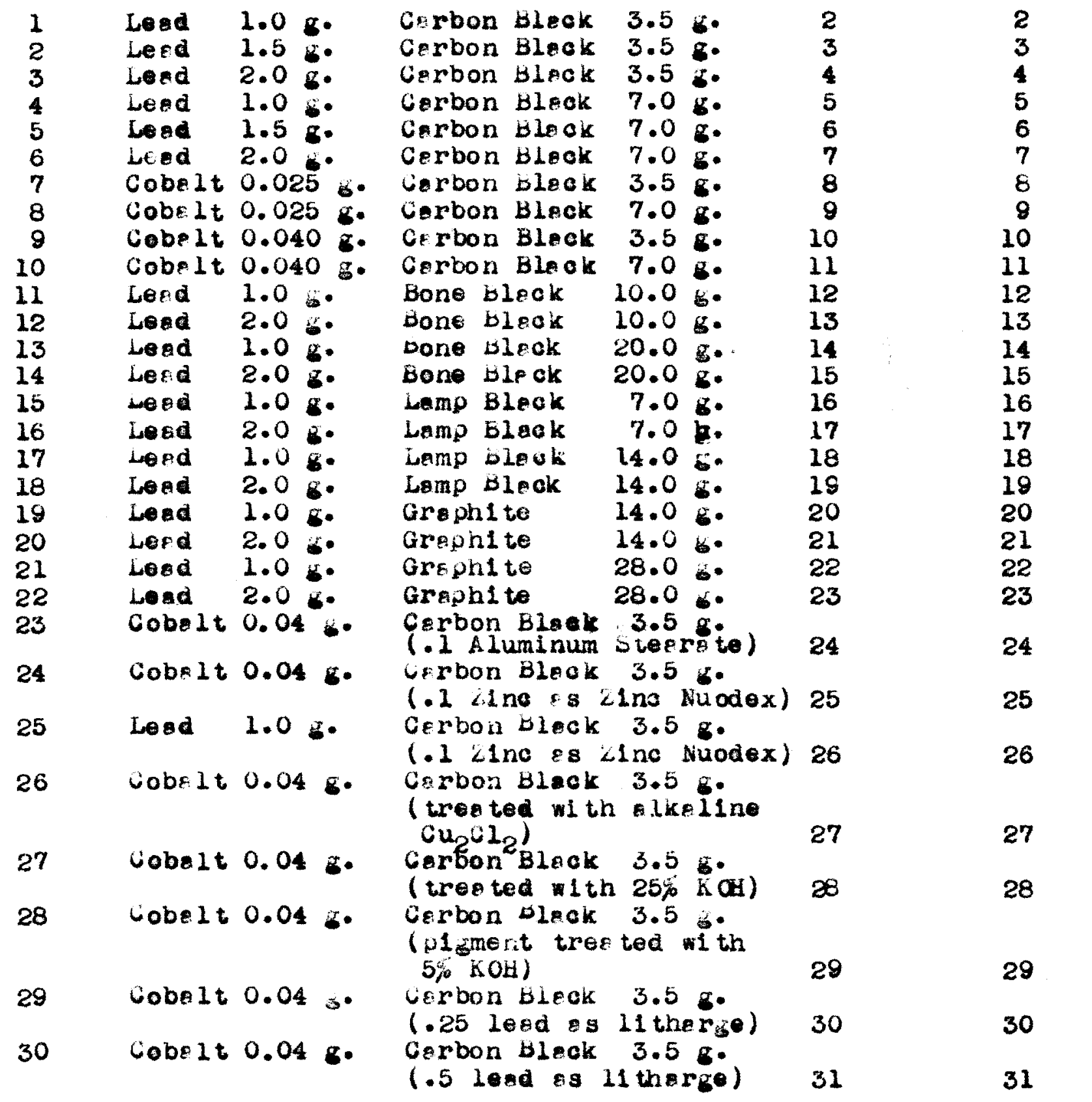

* The composition of ell mixtures ls expressed es perta of drier, a metel, na perts of plgment per 100 perts of IInseed oll. 


\section{Ioble 2}

Drying det for linsed ofl containing 1 grem lead na 3.5 grams esrbon blak per 100 gram o11. (Porcent 011, 83.0)

6 months 1 ing

at. cample, $.3452 \mathrm{~B}$. it. o11, .3205 g.
9 monthe exing

at. semple, .15i4 8 . it. ofl, $.1460 \mathrm{~g}$.
14 months $\operatorname{lng}$

it. smplo, .2392 g. it. 011 , .2220 g.
Lime, Inereese inereese
Hours In weignt, in welght orems
Inorese Increse

in welght, in wolght frms of 011

Increase inerese in weight, in weight erams of o11

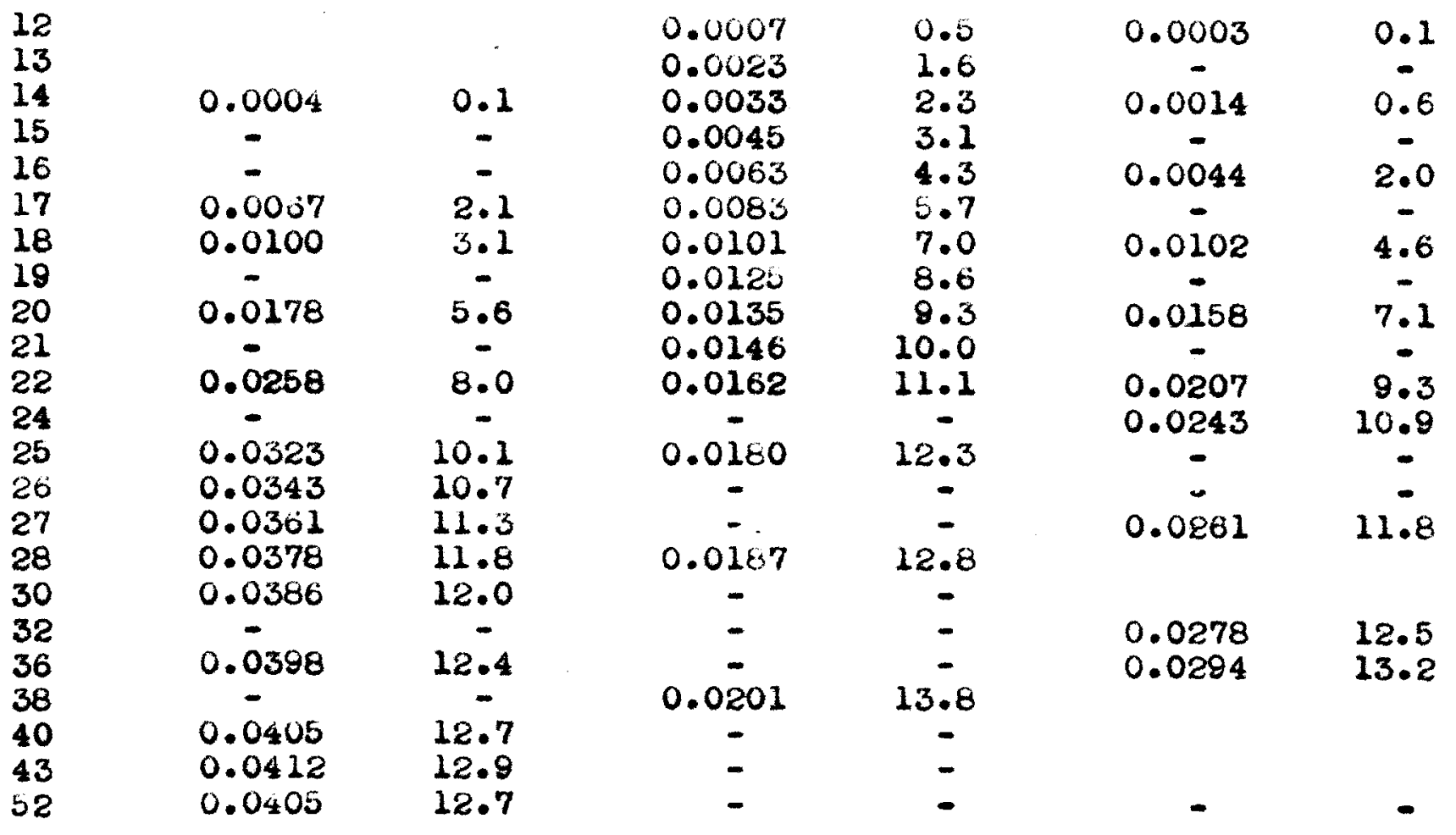


T.ble 3

Lrylng de te for linseed oll contelning 1.5 grems leed and 3.5 grame os rbon blfok per 100 grams oll. (Percent 011, 91.4)

\section{6 months ging}

wt. ssmple, .2996 g.

it. o11, .2738 E

1.0. Hours in weight, in weight grame of 011

$\begin{array}{ccc}8 & 0.0014 & 0.5 \\ 10 & 0.0075 & 2.7 \\ 11 & 0.0139 & 5.1 \\ 12 & - & - \\ 13 & 0.0217 & 7.9 \\ 14 & - & - \\ 15 & 0.0266 & 9.7 \\ 16 & - & - \\ 17 & - & - \\ 18 & 0.0279 & 10.2 \\ 19 & - & - \\ 20 & - & - \\ 21 & 0.0291 & 10.6 \\ 22 & - & - \\ 25 & - & - \\ 26 & 0.0305 & 11.2 \\ 27 & - & - \\ 28 & - & - \\ 31 & 0.0317 & 11.6 \\ 32 & - & - \\ 46 & 0.0320 & 11.7 \\ 49 & & \end{array}$

9 monthe $\ln \ln 8$

*t. smple, .1418 g. it. semple, .2219

at. o11, .1295 g.
14 monthe $\ln$ ing

t. o1l, .2025 8 .

$$
\begin{gathered}
0.0024 \\
0.0048 \\
0.0080 \\
0.0122 \\
0.0135 \\
0.0149 \\
0.0155 \\
0.0103 \\
0.0169 \\
0.0175 \\
0.0179 \\
0.0178 \\
0.0175 \\
0.0176 \\
- \\
0.0174 \\
0.0174 \\
- \\
0.0168
\end{gathered}
$$

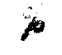
Increse of 011

$$
\begin{array}{r}
1.8 \\
3.7 \\
6.2 \\
9.4 \\
10.4 \\
11.5 \\
12.0 \\
12.6 \\
13.1 \\
13.5 \\
13.8 \\
13.7 \\
13.5 \\
13.6
\end{array}
$$

-

13.4

13.4

13.1
0.0028

0.0092

$0.0 \overline{152}$

0.0203

0.0229

0.0248

$0.0 \overline{255}$

-

0.0266

-

0.0266

0.0262
1.4

$-$

4.5

7.5

10.2

11.3

12.3

12.6

$-$

13.1

$-$

13.1

12.9 


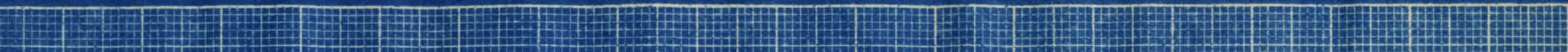

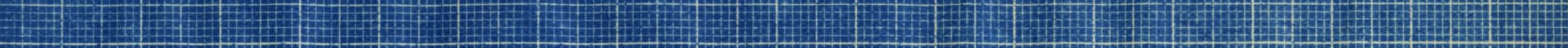

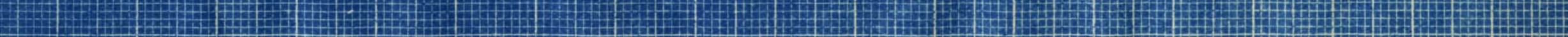
L L

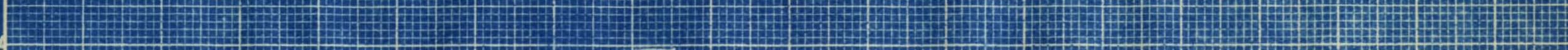

14

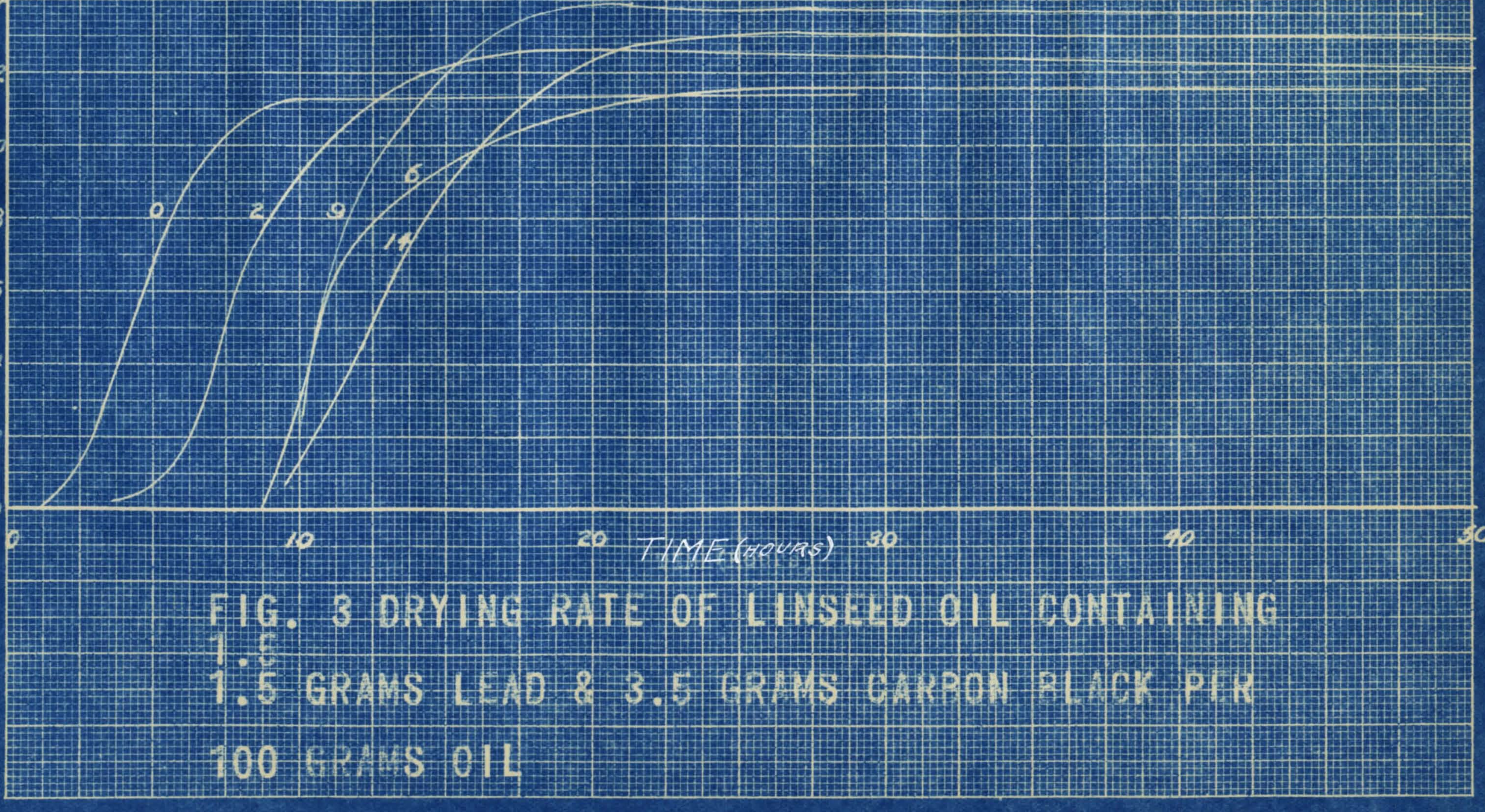


IADL 4

Drying de te for linseed oll conteinlng 2 frsm lefd $t$ nd 3.5 grems eerbon blick jer 100 grems 011. (Percent 011, 89.4)

6 months eqing

it. semple, .2102 8 . it. $011, .1880 \mathrm{~g}$.

Time, Increese Incresese Hours in wolght, in welght grems
9 months $8 \mathrm{~g} 1 \mathrm{ng}$

t. srmple, .1437 d in. 011 , .12838.

\section{4 months oging}

it. semplo, $.2468 \mathrm{~g}$. it. o11, .2205 $\mathrm{g}$.

\%

Inoresse incresse in weight, in weight orems of o11

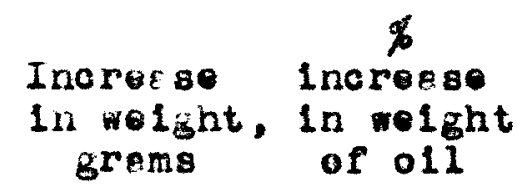

$\begin{array}{ccc}8 & 0.0024 & 1.3 \\ 10 & 0.0072 & 3.8 \\ 11 & 0.0157 & 8.4 \\ 12 & - & - \\ 13 & 0.0191 & 10.1 \\ 14 & - & - \\ 15 & 0.0209 & 11.1 \\ 16 & - & - \\ 17 & - & - \\ 18 & 0.0223 & 11.8 \\ 19 & - & - \\ 20 & - & - \\ 21 & 0.0228 & 12.2 \\ 25 & - & - \\ 27 & - & - \\ 31 & 0.0230 & 12.2 \\ 46 & 0.0224 & 11.9 \\ 49 & - & - \\ 53 & 0.0223 & 11.9 \\ 58 & - & -\end{array}$

$\begin{array}{cr}0.0099 & 7.7 \\ 0.0118 & 9.2 \\ 0.0141 & 11.0 \\ 0.0163 & 12.7 \\ 0.0166 & 12.9 \\ 0.0171 & 13.4 \\ 0.0173 & 13.5 \\ 0.0172 & 13.4 \\ 0.0172 & 13.4 \\ 0.0171 & 13.4 \\ 0.0171 & 13.4 \\ 0.0171 & 13.4 \\ 0.017 & - \\ 0.0169 & 13.2 \\ 0.0158 & 12.3\end{array}$

0.0101

4.6

0.0182

8. 2

0.022

10.1

$-$

0.0252

11.4

0.026

11.9

-

0.0272 -

$-$

$-$

$-$

11.9

$-$

0.0155

12.1 
Leble 5

Drying de te for lirseed oll conteining 1 grem loed and 7 grams cerbon bleck por 100 grams 011. (Percent 011, 89.9)

6 nonths 8 ining

t. $82 \mathrm{mplo,} .2614 \mathrm{~g}$. it. o11.
9 months 29115

it. semplo, $.1650 \mathrm{E}$. it. o11.

\section{$.1482 \mathrm{~g}$}

14 months 8 ing

t. semple, .2037 g. t. o11, .1830 $\mathrm{g}$.

Time, Incrosse increage Inorease increase Increase increase Hours in welght, in weIght in weight, in weight in weight, in weight grems of oil

brems of oil grems of 011

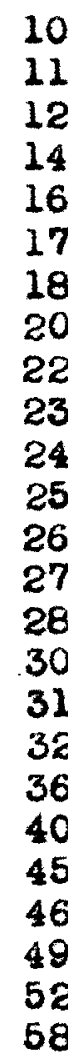

$\begin{array}{cc} & \\ & \\ 0.0006 & 0.3 \\ 0.0006 & 0.3 \\ 0.0006 & 0.3 \\ 0.0011 & 0.5 \\ 0.0022 & 0.9 \\ - & - \\ 0.0086 & 3.7 \\ 0.0128 & 5.4 \\ - & - \\ 0.0183 & 7.8 \\ 0.0222 & 9.5 \\ - & - \\ - & - \\ 0.0277 & 11.8 \\ 0.0291 & 12.4 \\ 0.0297 & 12.6 \\ - & - \\ 0.0297 & 12.6 \\ - & -\end{array}$

0.0003

0.0006

0.0022

0.0022

0.0024

0.0026

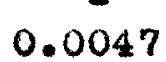

0.0082

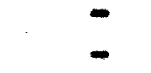

0.0136

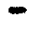

$-$

0.0167

0.0186

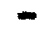

-

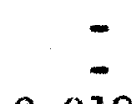

0.0194

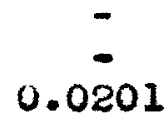

0.2

0.4

1.5

1.5

1.6

1.8

$-$

3.2

5.5

$-$

$-$

9.2

$-$

1.3

11.3

12.5

$-$

$-$

-

$13 \cdot 1$

13.6
0.0005

0.0005

0.0006

0.0014

0.0034

0.0062

$0.0 \overline{103}$

$-$

0.0154

-

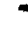

0.0203

0.0218

-

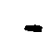

0.0229

$-$
0.3

0.3

0.3

$-$

0.8

1.8

3.4

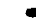

5.6

$-$

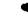

8.5

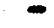

-

11.1

11.9

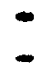

12.6

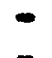




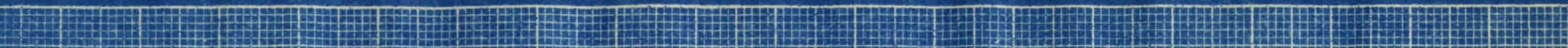
15: 2. 1: 1 [1+ | 1.

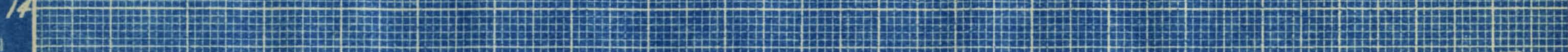
1. 
Teble 6

Drying de te for linseed oil conteining 1.5 grems leed end 7 grems cerbon bleok per 100 grems 011. (Percent 011, 88.3)

$$
\begin{aligned}
& 6 \text { months eglng } \\
& \begin{array}{l}
\text { it. semplo, } .2319 \mathrm{~g} \text {. } \\
\text { it. o11, } .2043 \mathrm{~g} \text {. }
\end{array}
\end{aligned}
$$

9 months eglng

it. sample, .1265 g. w. 011. .1118 g.
14 months Aging

"t. semple, .2404 g. w. oll, .2122 g.

Time, Increase incresso Incresse increase Increrse increase Hours in weight, in weight in weight, in weight in weight, in weight grems of of 1 grans of 011 grems of oli

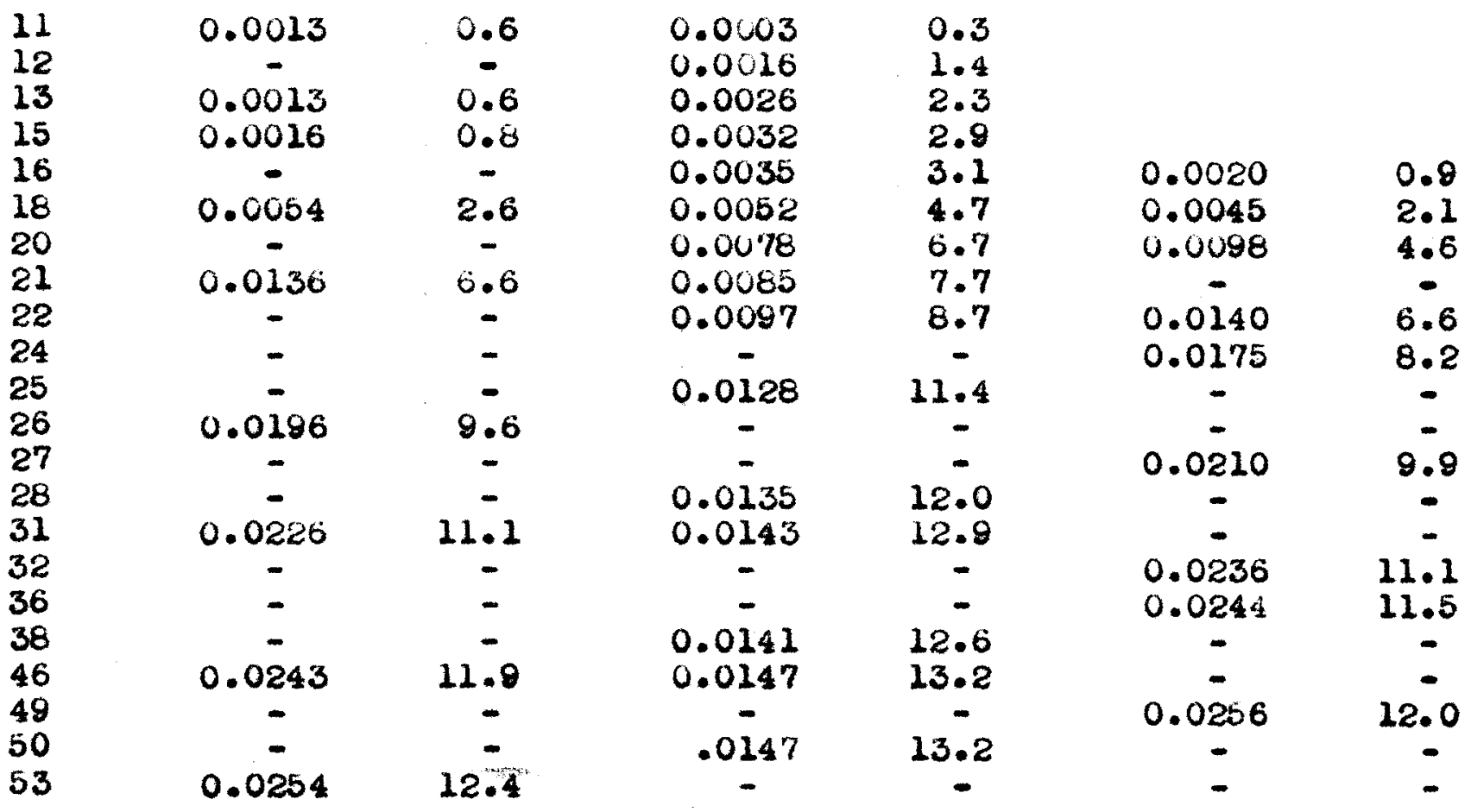




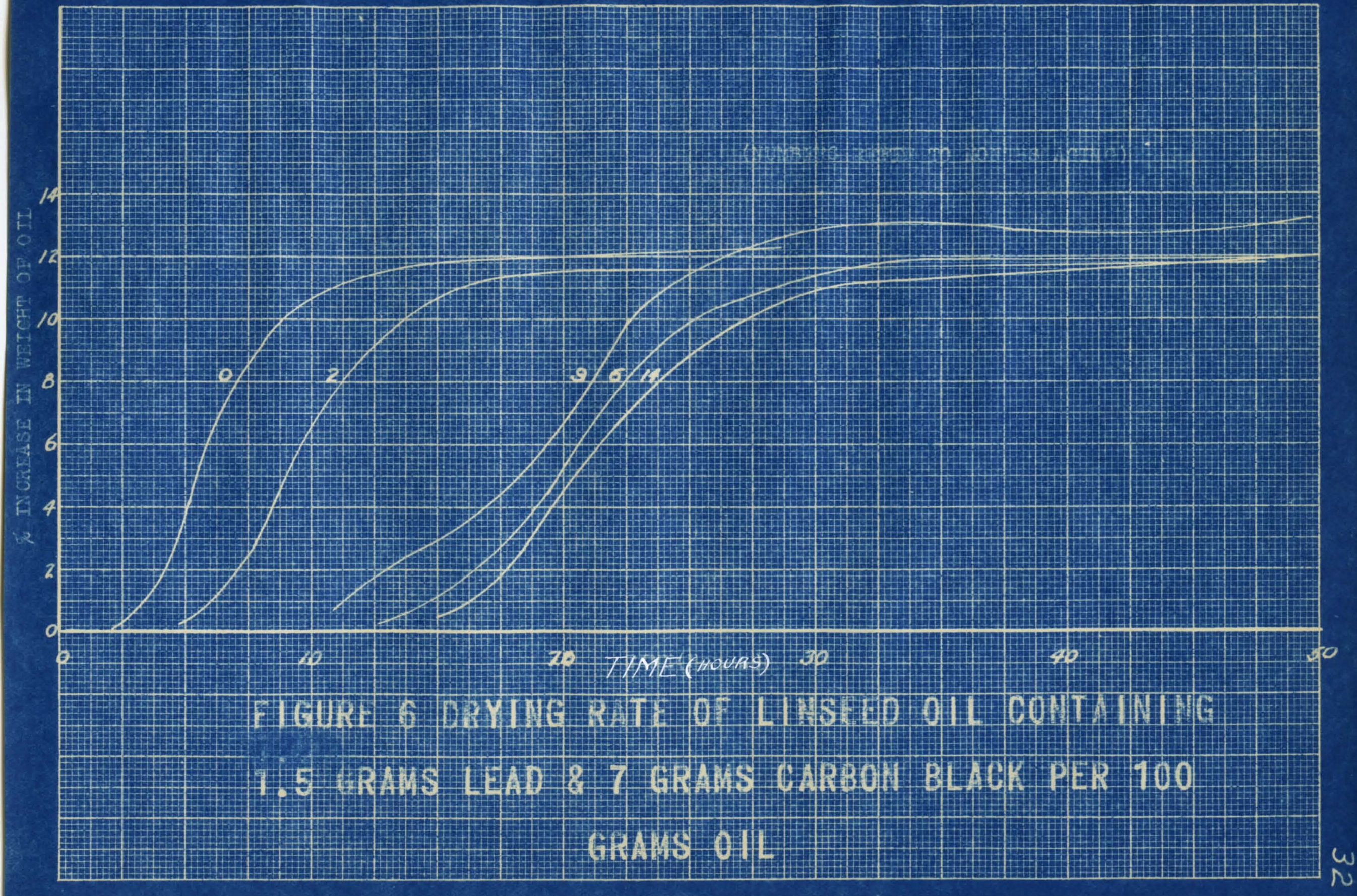


Ieble 7

Urying de te for linseed oll conteining 2 grims leed and 7 grams cerbon bleck per 100 grams 011. (Percent 011, 86.8)

6 months eging

wt. semplo, .2928 8 it. o11, .2540 $\mathrm{g}$

8

Time, Increese Inorese Hours in welght, In welght grams of o1 1
9 months 8 ging

st. semple, .1272 8 . it. 011 . . $1100 \mathrm{~g}$.
Inos

Inoreaso in weight, rems

\section{4 months aging}

wt. semplo, .2265 $\mathrm{g}$. it. o11, $.1965 \mathrm{~g}$.

inereose in welght of o11
1

Inorease Increase in welght, in welght crems of 011

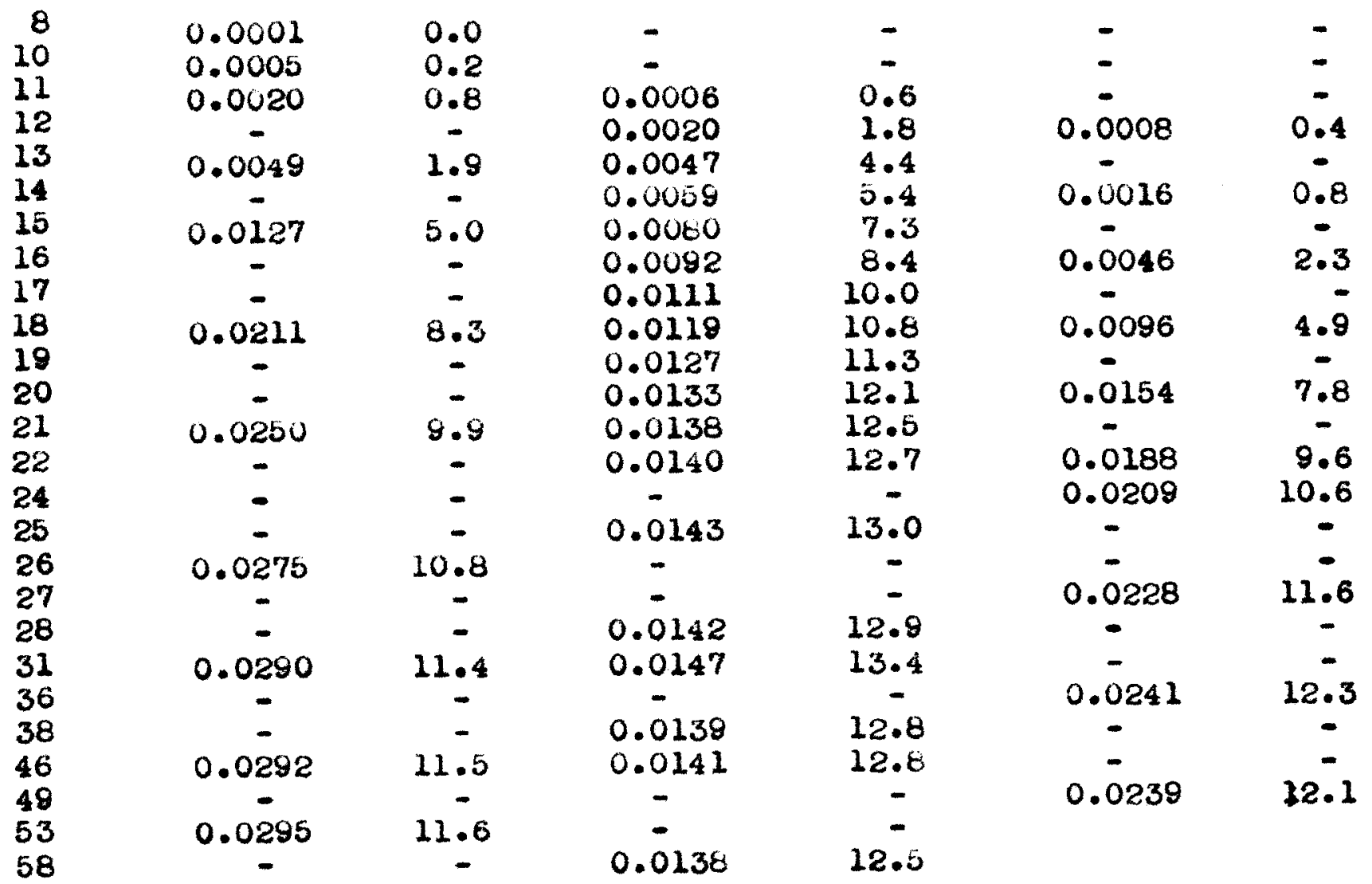




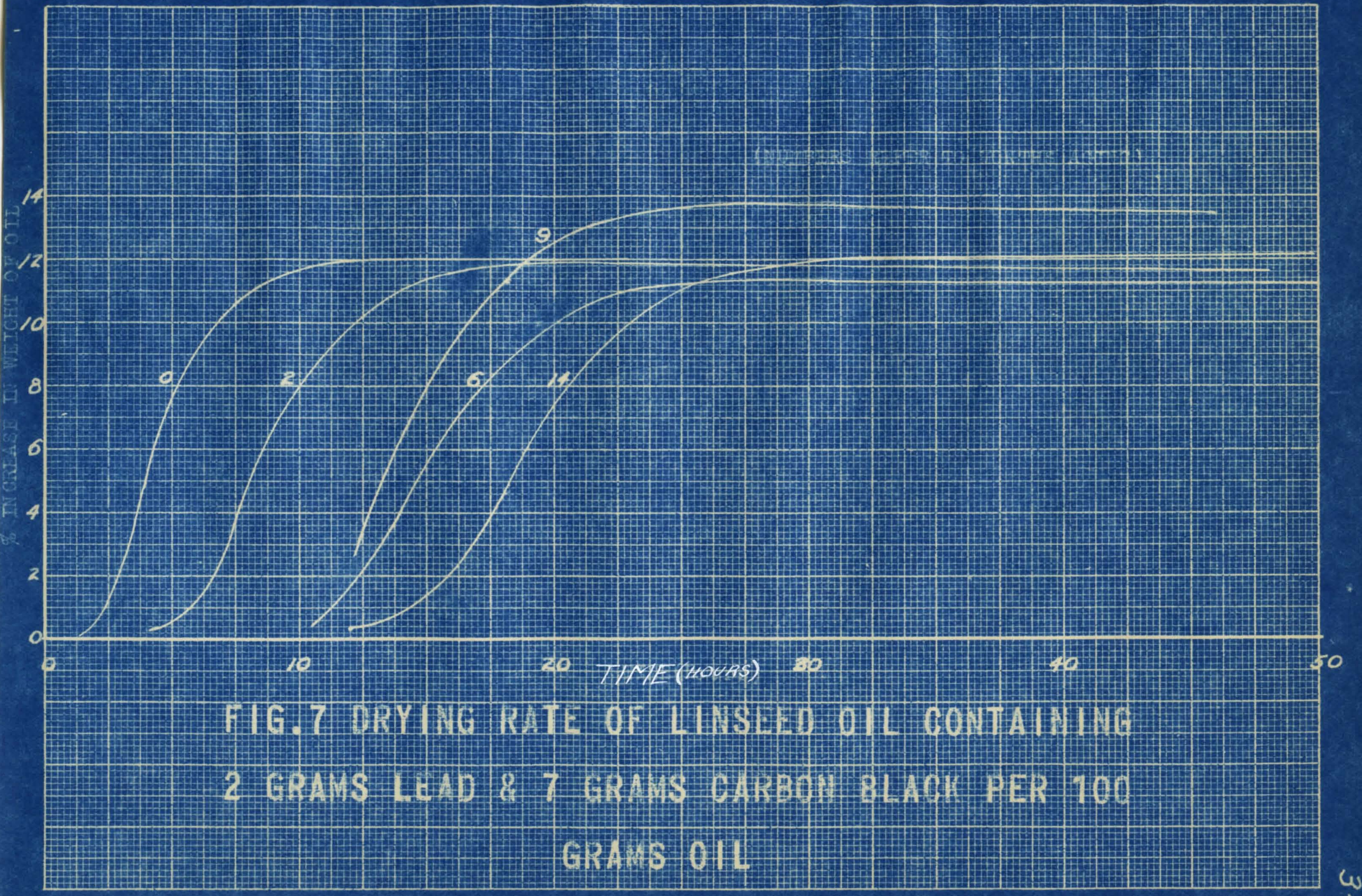


Table 8

Drying de te for $11 \mathrm{nseed}$ oll conteining 0.025 grem cobelt end 3.5 grems cerbon bleok per 100 grems o11. (Pereent 011, 96.4)

6 months golige

it. semple, .2952 8 . it. 011 .
9 monthe eging

wt. semple, .2148 B. t. oll, .2070 $\mathrm{B}$.
14 months $\operatorname{ging}$

"t. semple, .2459 g. *t. 011, .2368 b.

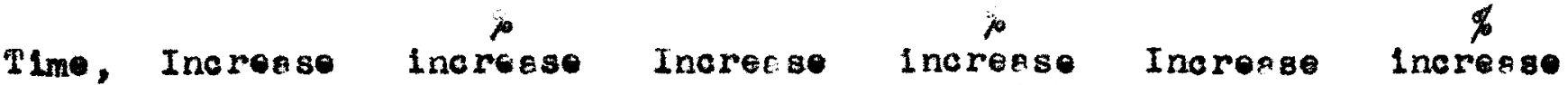
Hours in weight. In weight in weight, in weight in weight, in weight arems of oil Srems of 011 grems of o11

11

12

13

14

15

16

18

20

22

24

25

27

30

32

36

38

41

45

46

49

50

52

55

58

60

68

85
0.0005

0.006

$-$

-

0.0015

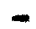

-

0.0068

0.0122

0.0204

0.0317

0.0378
0.2

0.2

-

-

$-$

0.5

-

-

$-$

2.4

$4 \cdot 3$

$-$

7.2

11.1

13.3
0.0004

0.0022

0.0022

0.0022

0.0019

0.0018

0.0020

0.0020

0.2

1.1

1.1

1.2

0.8

0.9

1.0

1.0

0.0024

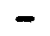

$-$

$-$

0.0028

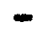

$-$

0.0077

-

0.0118

$-$

0.0238

-

$0.0 \overline{0} 05$

1.2

-

-

$-$

1.4

-

$-$

3.7

$-$

5.8

$-$

$-$

11.5

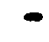

$-$

14.8
0.0006

0.0006

0.0006

0.0006

0.0031

0.0050
$0.0 \overline{0102}$

$0.0 \overline{2} 22$

0.0280

0.0321

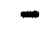

0.0321
0.3

$-$

0.3

0.3

0.3

1.3

2.1

4.3

9.4

12.3

13.6

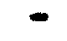

13.6 


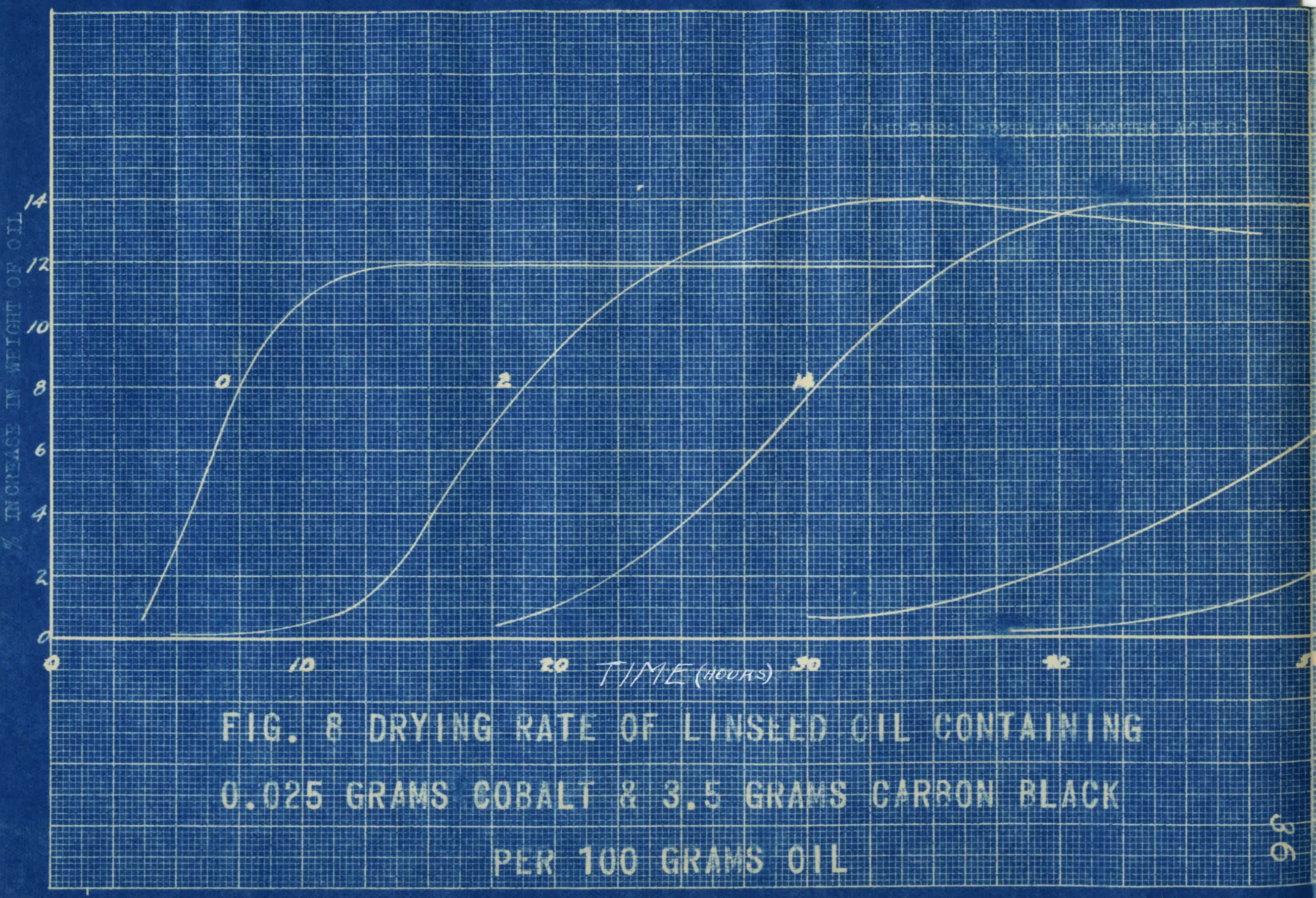




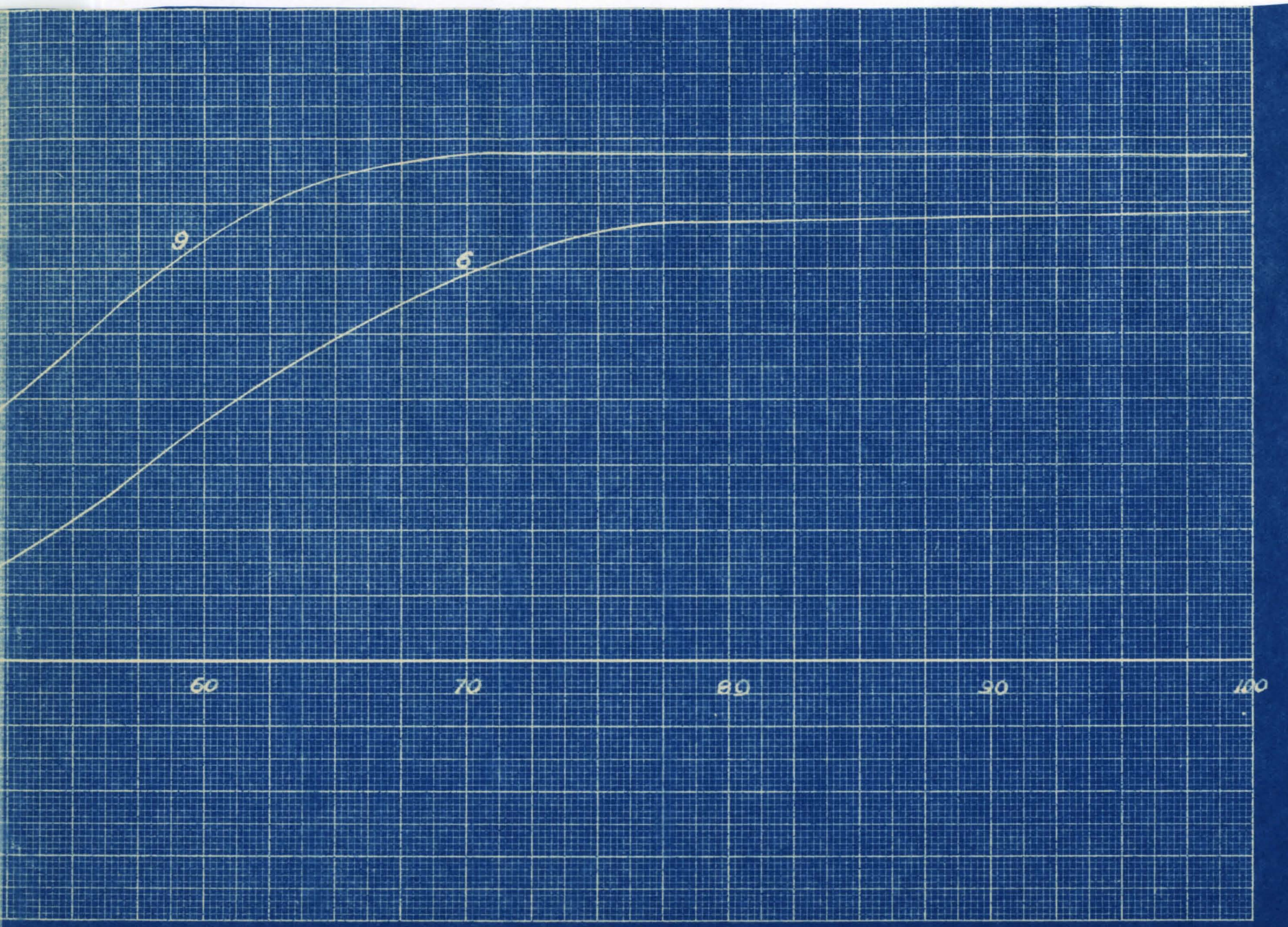


Teble 9

Drying de te for 1 inseed ofl containing 0.025 grem cobelt nnd 7 grams of rbon bleck per 100 grams oll. (Percent 011, 93.2)

6 months exing

wt. SEMPLE, .2658 is.

w. oll, .2660 g.

I Imo,

Hours

12

13

14

15

16

18

19

20

21

22

25

28

32

34

42

45

46

50

52

55

58

60

69

85

108

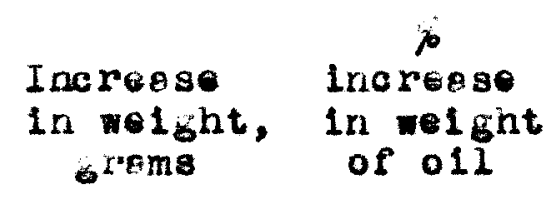

\section{9 months efins}

wt. semple, .1728 $\mathrm{g}$.

it. $011, .1610 \mathrm{~g}$.

$\begin{array}{ll}\text { Increase } & \$ \\ \text { in welght, } & \text { in wereese } \\ \text { grems } & \text { of oll }\end{array}$

$\begin{array}{cc}0.0007 & 0.4 \\ 0.0020 & 1.3 \\ 0.0019 & 1.2 \\ 0.0022 & 1.4 \\ 0.0020 & 1.3 \\ 0.0027 & 1.7 \\ 0.0031 & 1.9 \\ 0.0030 & 1.9 \\ 0.0028 & 1.8 \\ 0.0035 & 2.2 \\ 0.0038 & 2.4 \\ 0.0042 & 2.6 \\ 0.0049 & 3.0 \\ 0.0055 & 3.5 \\ 0.0068 & 4.2 \\ - & - \\ 0.0078 & 4.9 \\ 0.0085 & 5.3 \\ - & - \\ 0.0109 & - \\ - & 6.8 \\ 0.0232 & -- \\ 0.0225 & 14.4 \\ 0.0\end{array}$


leble $9-A$

Lrying de te for 11 resed oll contelning 0.025 grem cobelt end 7 grems cerbon bleck per 100 grams o1l. (Pereent 011, 93.2)

\section{4 moriths $8 \mathrm{~g} \operatorname{lng}$}

*t. sample, .2120 g.

t. t. o11, .1875 8.

Ime,

Hours

5

6

7

9

10

11

13

16

21

22

25

27

32

36

38

41

49

62

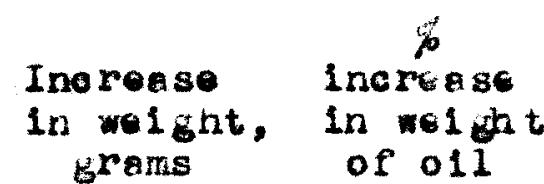

hod 1 yes

tepround

Wt. sample, .2083 $\mathrm{g}$. int. oll, $.1940 \mathrm{~g}$.

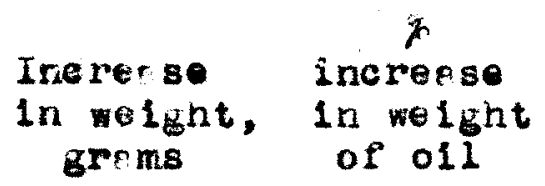

$\begin{array}{cc}0.0021 & 1.1 \\ 0.0031 & 1.6 \\ 0.0051 & 2.6 \\ 0.0105 & 5.4 \\ - & - \\ 0.0150 & 7.7 \\ 0.0182 & 9.8 \\ 0.0235 & 12.1 \\ 0.0257 & 13.2 \\ - & - \\ 0.0256 & 13.2 \\ - & - \\ - & - \\ - & - \\ 0.0244 & 12.6\end{array}$




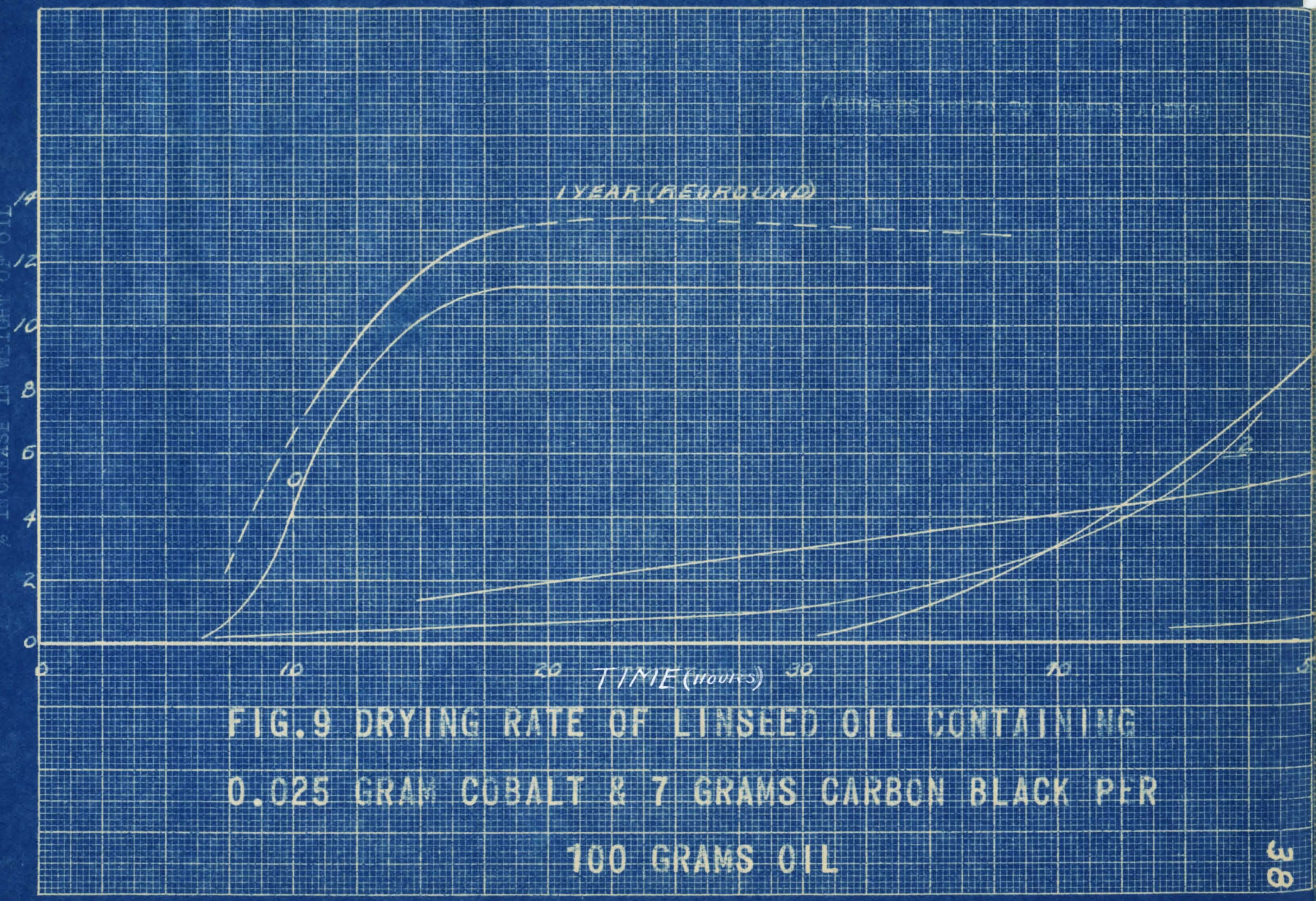


liebie 10

Lrylng dete for linseed ofl conteining 0.04 grem cobelt end 3.5 grems cfrbon black per 100 grams o11. (Percent 011, 96.2)

6 monting $E$ in

*t. semple, $.2455 \mathrm{~g}$.

itt. 011, .2360

Ime,

Hour's

12

14

16

20

28

31

33

38

40

43

45

46

50

52

55

58

60

69

85

108

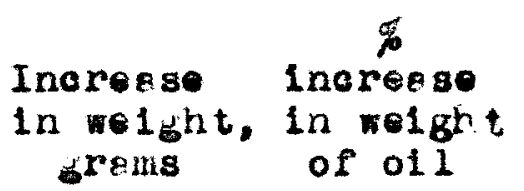

$$
\text { orems of oil }
$$

8 months eging

it. semple,.2244 8 .

it. 011, .2160 g.

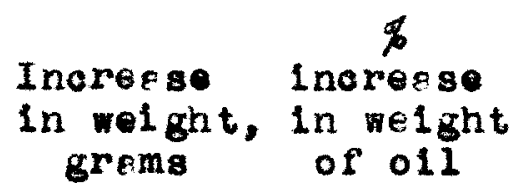

$\begin{array}{cc}0.0006 & 0.3 \\ 0.0018 & 0.8 \\ 0.0017 & 0.8 \\ 0.0017 & 0.8 \\ 0.0017 & 0.8 \\ 0.0020 & 0.8 \\ - & - \\ 0.0023 & 1.1 \\ - & - \\ - & - \\ .0030 & 1.4 \\ .0037 & 1.7 \\ - & - \\ - & - \\ 0.0061 & 2.9 \\ - & - \\ - & - \\ 0.0223 & 10.3 \\ 0.0202 & 8.4\end{array}$


Teble 10-A

Lrying de te for llnses ofl conteint 0.04 frem cobelt and 3.5 grems cerbon bleok per 100 grems o1l. (Porcent 011, 96.2)

14 montins Eping

iit. stmple, .2271 g.
it. oll, $.2183 \mathrm{ge}$

Time,

Hours

2

3

4

5

6

7

8

9

12

15

22

24

27

32

36

41

48

1.0

2.5

6.0

11.7

13.3

13.6

13.3
Aged 1 yerr Feground

Ht. semple, $.1877 \mathrm{~g}$. ot. o11, .1802 g. $\%$
Inoreese increese
in wolght, in weight
groms
0.0005
0.3
0.0002
0.0001
0.0005
0.2
0.0012
0.0019
0.0054
0.0089
0.0183
0.0256
0.0
0.3
0.7
1.0
3.0
4.9
10.2
14.2 


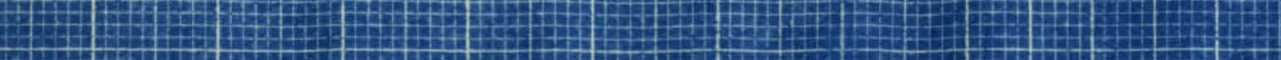

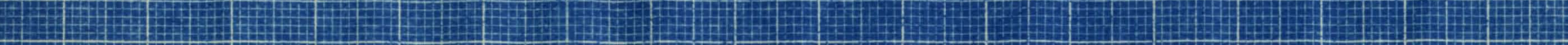

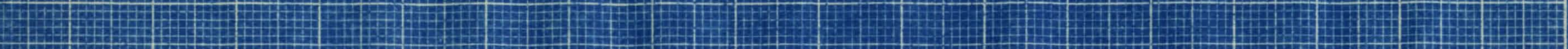

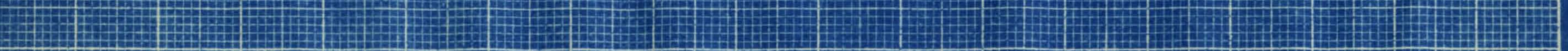

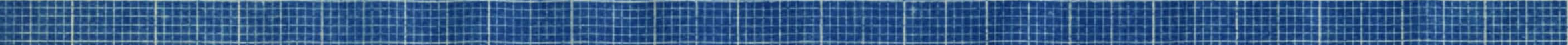
L 1 2. H H

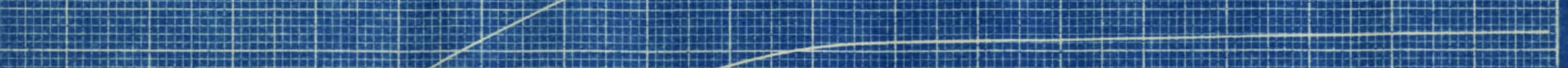
I 1

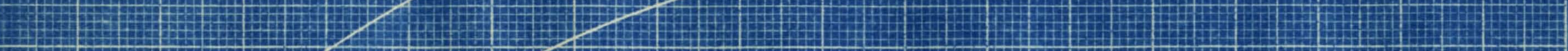
F 1

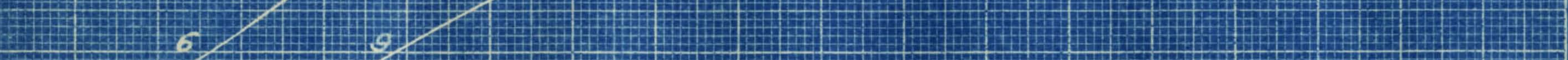

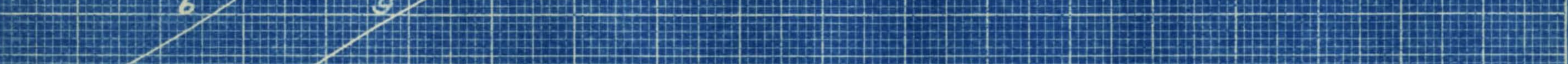

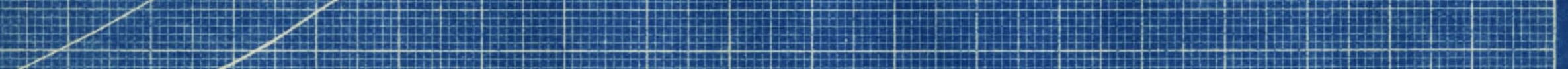

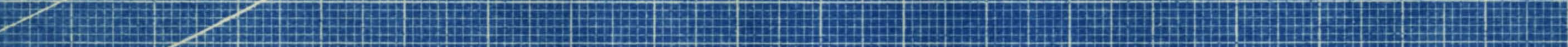
LI T P

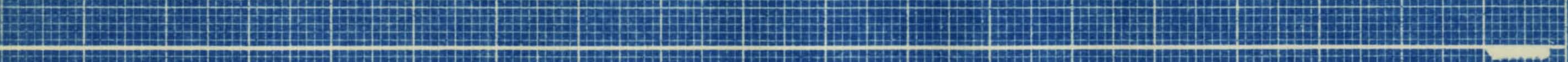

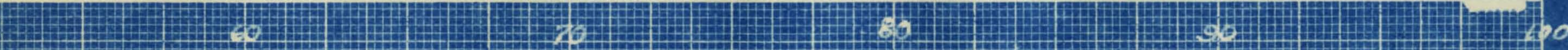
F. F.

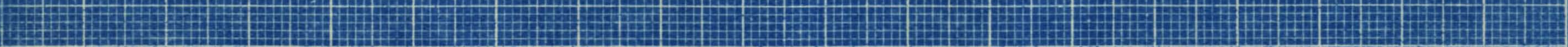
2. L.

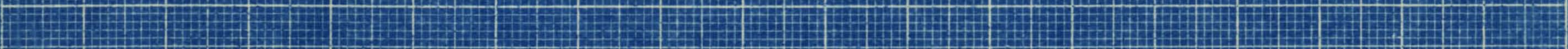
\begin{tabular}{|l|l|l|l|l|l|l|l|l|l|}
\hline & &
\end{tabular} 
Teble 11

Drying de te for linseed oll conteining 0.04 grem cobelt and 7 grems cerbon bleak per 100 grems o1l. (Percent 011, 93.0)

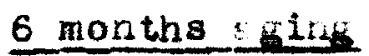

t. semple, .2768 s.
t. oil,
.2570 .
9 months fing

I'ime, Hours
Inorease Increase increase
in welight, in weight
grems of oil

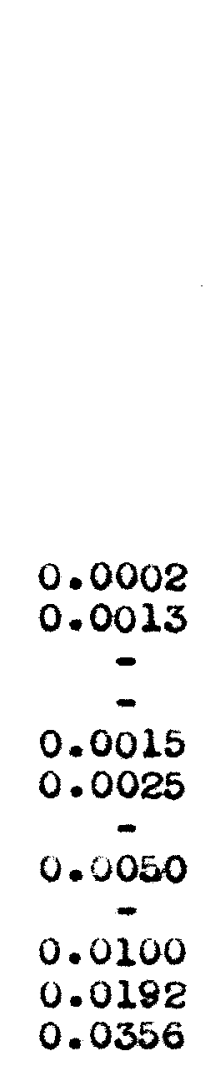

11

12

13

14

15

17

19

21

22

24

28

32

38

40

45

49

50

52

55

58

60

62

69

85

108

0.2

0.5

$-$

$-$

0.6

$-$

1.9

3.9

7.5

13.8

it. seinp le, $.3957 \mathrm{~g}$

"t. o11,

14 months eging

it. semple, .2119 g. iit. 011, .1970

$\%$ Increese incrense Increese Incresse in ight, in weight grems of ofl

in wolght, in welght groms of 011 


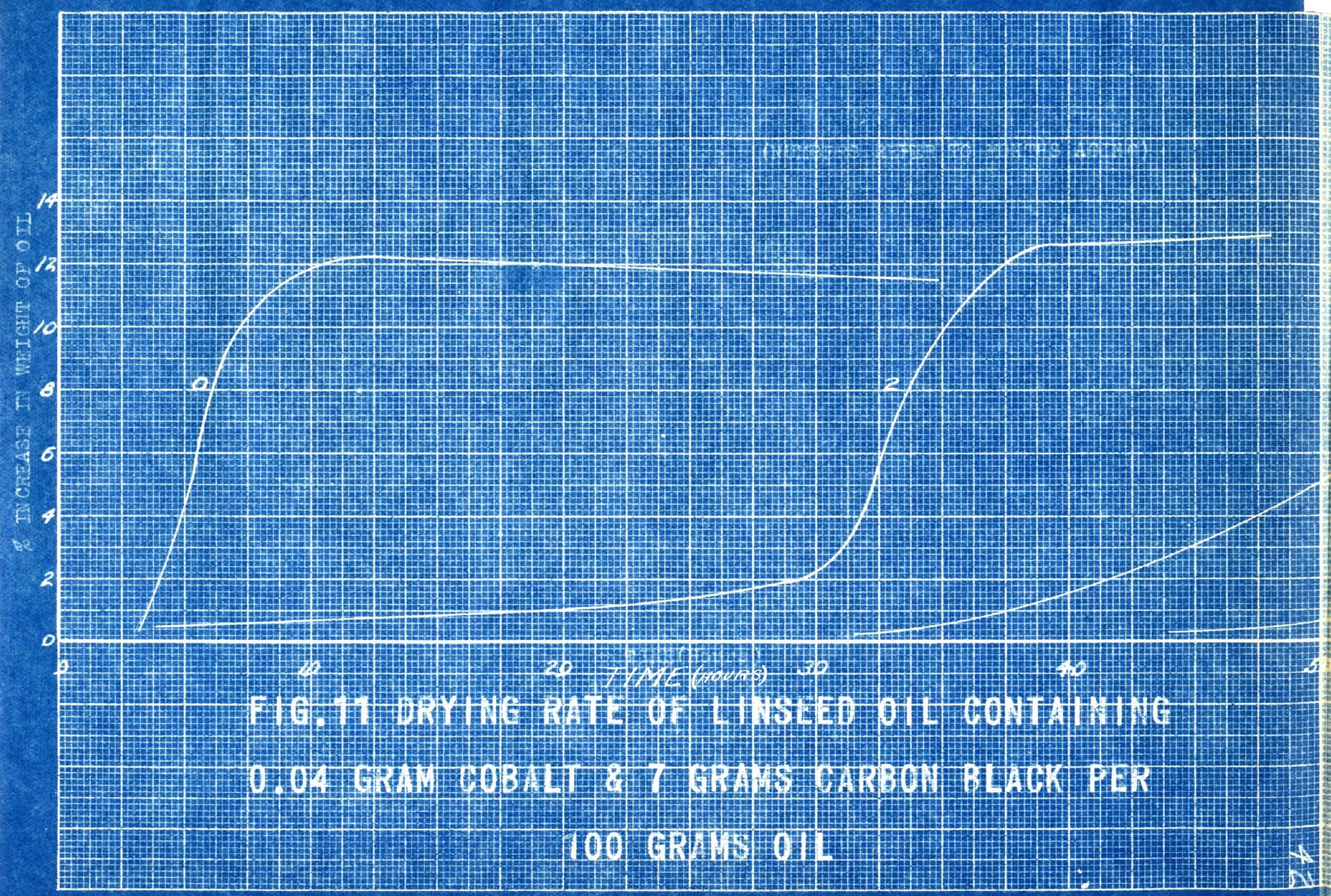


1. [1.

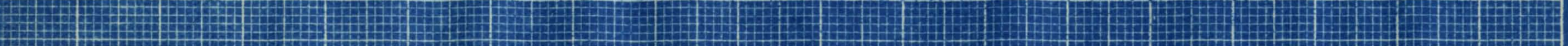
|

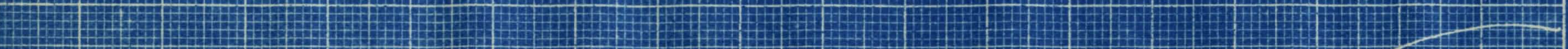

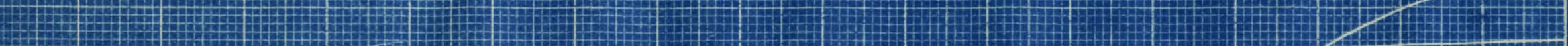

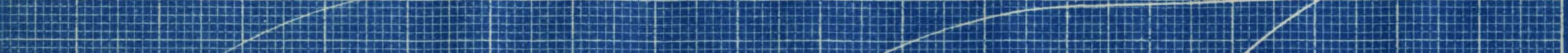

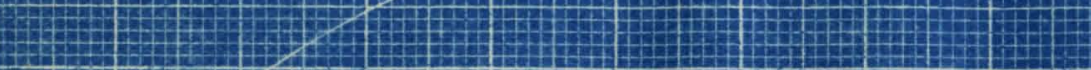

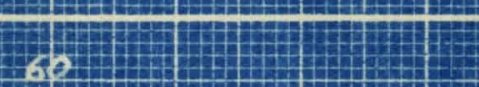

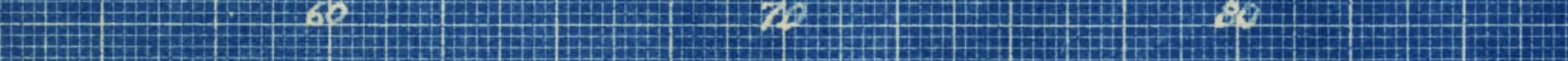


BONE BLACK 
It was demonstreted by Flgure 12 (1 grem lesd fnd 10 grems bone bleck per 100 grems o11) thet the induction period of the freshly prepered ample was fairly large, being twelve hours, with the sixth month's ourve belng. maximum et twenty-seven hours. The ninth end fourteenth month's both hed Induction perlods of twonty-s1x hours. The se gemples hod o sme 11 or drylng rete, thet is, required - longer time for the gein in weight to reach meximum, than other prev lous sem ples.

The Incress in drier coneentretion, 9 shown in Figure 13 (8 grems leed end 10 grems bone bloce per 100 grams o11), caused smeller loss in drylng power end geve thorter induetion perlod, for the freshly mede sample, five hours. The meximum induction perlod wes twenty-three hours (fourteenth month). The arying re: tes for this somple wes distinotly greeter then those of stgure 12.

Figure 14 ( 1 grem lead and 20 grams bone bleck per 100 frems oll) and Flgure 15 (2 grems lead end 20 grams bone bleck per 100 grams o11) showed that the increese In bane bleck sontent did not ceuse eny greet loss in drying ebility es wes the cese of cerbon bleck. All amples in both figures had induction porlods lying botwoen ten and twenth hours. The increesed drier concentretion shown in Figure 15 did not notleesbly shorten the induetion period. 
The bore bleck mixtures il hed felrly long laduction perlods even for freshly medo semples. In the samples shown In Figures 12, 14 , snd 15 the retes of erying were sme ller then tho se of the semples of prevlous fllgures. The drylng petes of the simples of Flare 13 were simllar to most of the cerion bleck semples. The inorefse in plument content had very litule efleot on loss in drying power. 
Toble 12

Drylng de te for 11 nseed oil contelning 1 grem lead and 10 grems boneblock per 100 grems 011. (Percent 011, 87.6)

6 months Eking $\quad 9$ inonths sings $\quad 14$ months eging

it. semple, $.3348 \mathrm{~g}$. wt. semple, $.2189 \mathrm{~g}$. wt. s:mple, $.2857 \mathrm{~g}$. "t. 011, .2935 $\mathrm{g}$. wt. 011, .1905 g. it. 011, .2580 g.

Time, Inorese incresse Incresse increese Increese incresso Hours in weight, in 4 ght in welght, in weight in weight, in wolght grems of 011 greme of oll grems of oll

\begin{tabular}{|c|c|c|c|c|c|c|}
\hline $\begin{array}{l}11 \\
12 \\
13 \\
14 \\
18\end{array}$ & & & $\begin{array}{l}0.0003 \\
0.0010 \\
0.0018 \\
0.0024 \\
0.0026\end{array}$ & $\begin{array}{l}0.2 \\
0.5 \\
0.9 \\
2.2 \\
1.4\end{array}$ & $\begin{array}{c}0.0003 \\
0- \\
0.0004 \\
0.0 .09\end{array}$ & $\begin{array}{l}- \\
0.1 \\
0.1 \\
0.3\end{array}$ \\
\hline 23 & 0.0005 & 0.2 & $=$ & $=$ & $0.0 \overline{0} 23$ & 0.9 \\
\hline $\begin{array}{l}24 \\
25\end{array}$ & $0.0 \overline{0} 09$ & 0.3 & 0.0028 & $\overline{1.5}$ & $\begin{array}{c}0.0023 \\
-\end{array}$ & 0.9 \\
\hline 27 & 0.0032 & & - & - & 0.0038 & 1.5 \\
\hline $\begin{array}{l}28 \\
30\end{array}$ & $\begin{array}{l}0.0032 \\
0.0050\end{array}$ & $\begin{array}{l}2.1 \\
1.7\end{array}$ & 0.0040 & 2.1 & $\overline{-}$ & - \\
\hline 31 & 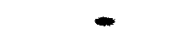 & - & 0.0062 & 3.3 & - & \\
\hline 32 & - & - & - & - & 0.0095 & 3.8 \\
\hline $\begin{array}{l}36 \\
38\end{array}$ & 0.0122 & 4.1 & 0ina & $\therefore$ & 0.0135 & 5.4 \\
\hline 41 & & & - & - & 0.0198 & 7.8 \\
\hline $\begin{array}{l}42 \\
43\end{array}$ & 0.0219 & 7.5 & 0.0167 & 8.8 & - & \\
\hline $\begin{array}{l}45 \\
46\end{array}$ & & & 0.0185 & 10.1 & - & \\
\hline $\begin{array}{l}49 \\
50\end{array}$ & & & 0.0216 & 11.4 & - & 1.0 \\
\hline & 0.0308 & 10.5 & & & & -1 \\
\hline & 0.0387 & 13.2 & 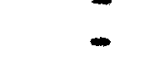 & - & 0.0334 & 13.4 \\
\hline & 0.0409 & 13.9 & 0.0249 & 13.0 & & \\
\hline
\end{tabular}




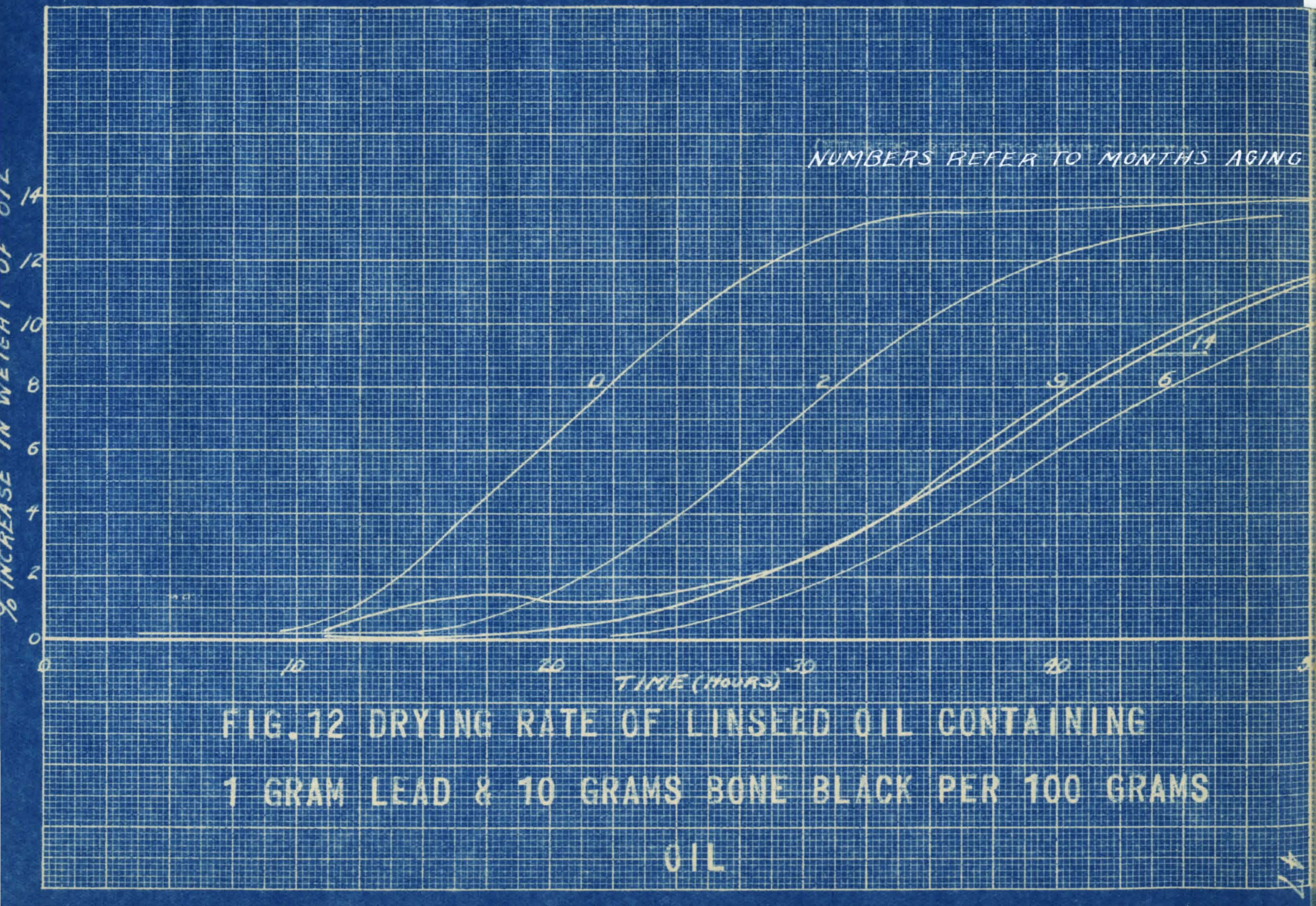




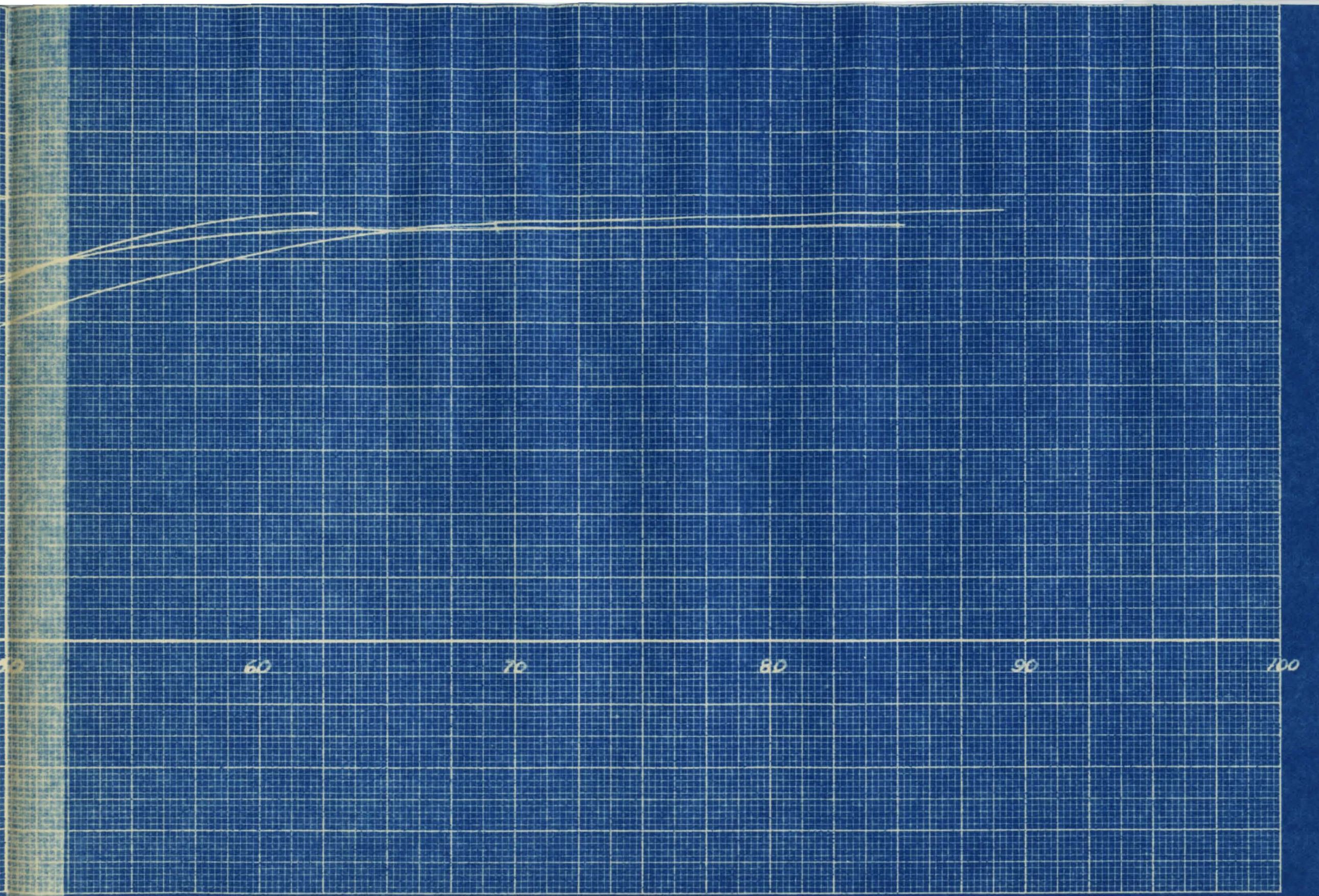


Tablo 13

Drying de te fo. $11 \mathrm{r} s 00 \mathrm{~d}$ oll contrinling 2 frems lea and 10 grams bone bleck per 100 grams o11. (Percent 011, 84.6)

6 months 8 ing

it. $88 \mathrm{mplo}, .2018 \mathrm{~g}$

it. 011 . Time, Inereese Increese
Hours in weight, in welght grams

of 011
9 months esing

w. sample, .2535

t. $011, \quad .2140 \mathrm{~g}$. Inorese

Incresse increase inelght, in welght
grams of oll
14 months esing

at. semple, .2628 $\mathrm{B}$. it. 011, .2220 g. Inoreese increese in wight, in wolght grems of o11
11

12

13

14

16

17

18

20

22

23

24

25

26

27

28

30

31

32

33

36

38

40

45

48

50

52

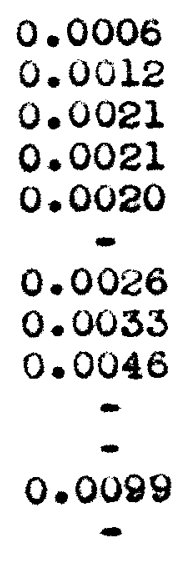

0.0101

0.0203

0.0240

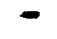

0.0272

0.0295

0.0307

0.0315

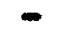

$-$

0.0314
8. 2

0.0
-
0.3
0.5
0.7
1.9
2.8
-
5.3
6.5
-
8.8
9.7
-
-
11.0
12.0
12.4
12.8
-
12.7

0.3

0.6

1.0

1.0

0.9

$-$

1. 2

1.5

2.2

$-$

4.6

$-$

7.6

0.0224

-

-

$-$

0.0273

-

$-$

0.0284

$-$

10.5
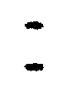

12.8
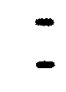

13.2
0.0005

0.0005

0.0005

0.0012

0.0024

0.0040
0.0076

0.0142

0.2

0.2

0.2

$-$

0.5

1.1

1.8

3.4

$-$

6.4

$-$

$-$

$0.0239 \quad 10.8$

$0.0273 \quad 12.3$

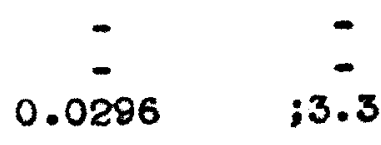




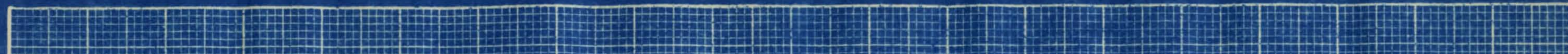

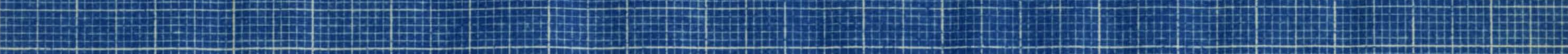
L -

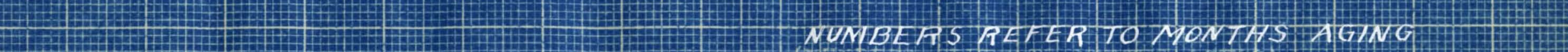
2. 1.

MT: 1 1 . GrMs OHL 
Teble 14

Wrylng do to for linseed oll containing 1 gram lead fand 20 grems bone bleck per 100 grems oil. (Percent oll, 80.5)

6 months oging

t. semple, .1562 है.

it. oll, $\theta$ morths $\varepsilon$ ging

$\begin{array}{ll}\text { t. sample, } & .2306 \mathrm{~g} \cdot \\ t .011, & .1855 \mathrm{~g} .\end{array}$
14 months 8.8 ing

"t. semple, .2481 g. t. o11. $.1895 \mathrm{~K}$.

Time, Irerease incresse Incresse increase Inoreese incream Hours in welght, in woight in weight, in weight in we ight, in waight grems of o1 grams of oll grams of 011

10

11

12

14

16

17

18

19

21

22

23

24

25

26

27

28

30

31

32

36

38

40

45

46

49

50

52

62

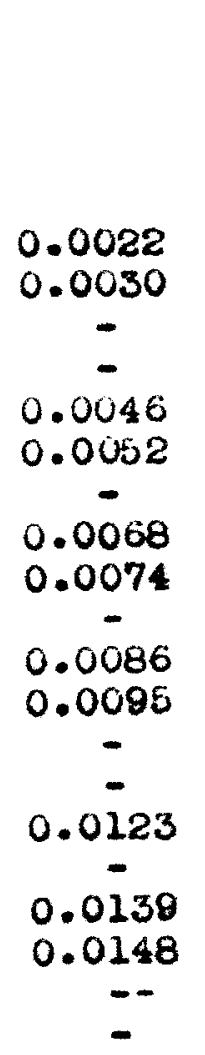

0.0157
0.0004<smiles></smiles>

0.0012

0.0023

0.0041

1.7

2.4

$-$

3.7

4.2

5.4

5.9

6.8

7.6

$-$

$-$

9.8

11.0

11.8

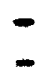

-

$12 \cdot 5$

0.0063

-

$-$

0.0100

-

0.0219

-

0.0145

-

$-$

$0.0 \overline{189}$

0.0217

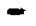

$-$

0.0245
0.2

$-$

0.6

1.2

2.2

$13 \cdot 2$

3.4

-

$-$

5.4

$6 \cdot 4$

$-$

$-$

7.8

-

$-$

$-$

$10 \cdot 2$

11.7

-

$0.0254 \quad 13.7$
0.0002

0.0010

0.0032

0.0051

0.0058

0.0066

0.0084

0.0097

0.0122

-

-

0.0145

-

$-$

0.0169

0.1

0.5

1.6

2.6

2.9

3.3

4.2

4.8

6.2

$-$

$-$

$7 \cdot 3$

$-$

8. 5

0.0286

$-$

9.3

$0.0 \overline{2}$

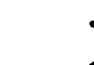

0.0262

0.0271

13.6 


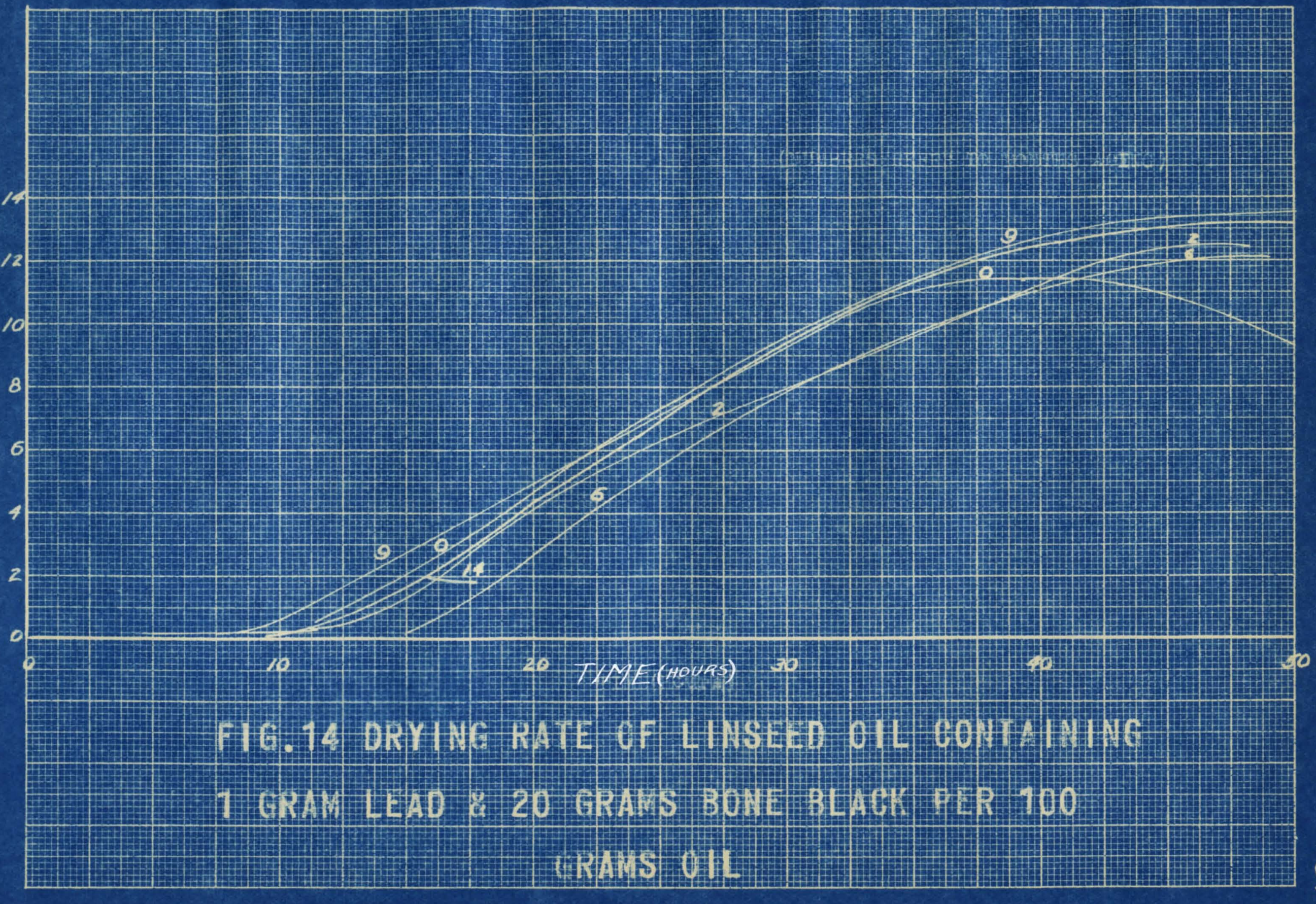


Toble 15

Drying ds to for linseed oil conteining 2 grems loed and 20 grams bone black per 100 grems oll. (Percent 011, 78.0)

6 months eglag 9 monthe ering 14 months aging

at. semple, .2344 g. "t. semple, .1683 g. wt. semple, .1951 g. t. 011, .1830 g. it. o11. .1310 g. it. 011, .1520 g.

Lime, Incresse Inctese Increase incresse Increase Incresse Hours in weight, in wolght in weight, in weight in weight, in weight grems of oil grams of oll grams of oll

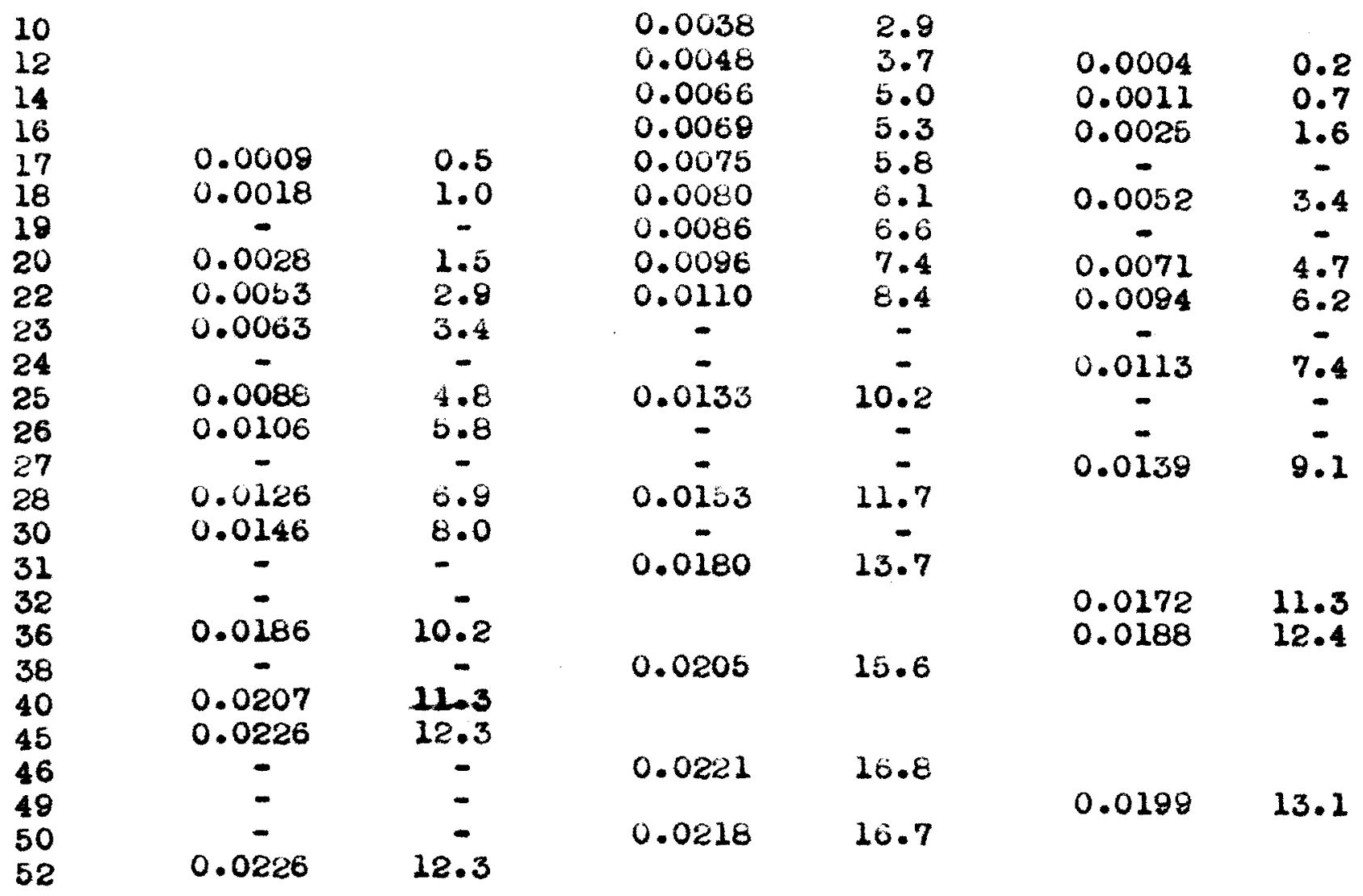


KEUFFEL \& ESSER CO., N. Y. NO, 350-11

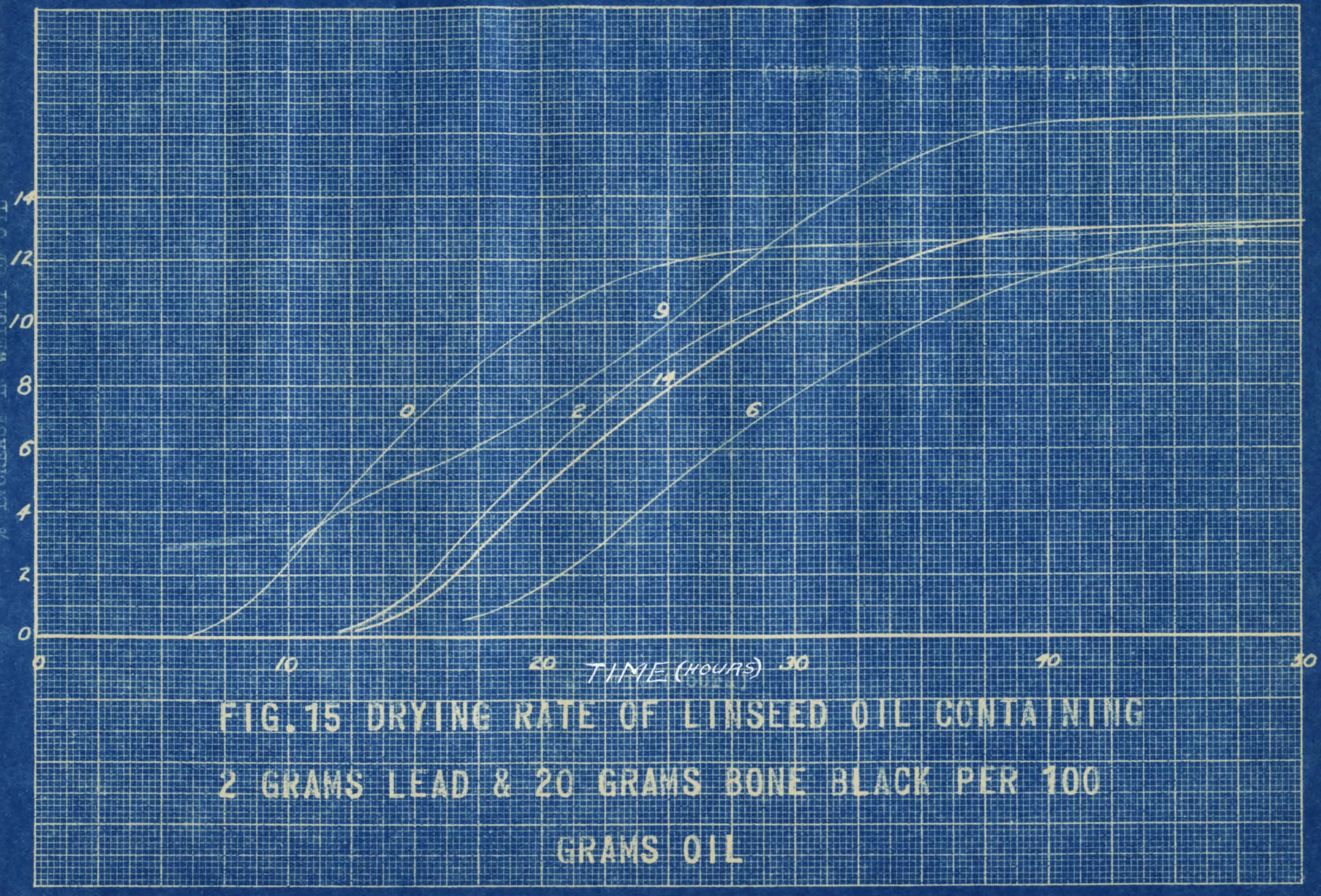


LANP BLACE 
Ihe semples of Flore 16 (1 grem Leed End 7 grems lemp bleck per 100 grems oll) exhiblted en induction perlod of four hours far the freshly made semple to meximum porlod of of ght hours (farteenth month).

Flgur 17 th semples of the composition 2 grems lead f nd 7 grems lamp glack or 100 groms oll indicated no decreese in tirie of Induction over the semples of rgure 16 The inc resed plgnent content of the semples of Flgure 18. contelning 1 grem leed and 14 groms lsmp block per 100 grems o1 crused a considerable loss in drying tims of this mix ture. The int tial induction perlod ws three hours whilo the meximum period we fifteen hours (fourteenth (nonth).

Theres a dstict decresse in laduction periods of the god samples of lgure 19 (2 grams lesd nd 14 grame Lemp bleok per 100 grems oll) over the corresponding semples of Flgures 18. This wes the result of ineresed drier content.

The lemp bleck semples 11 disple yed moderete 10880 In arying power exoept for those of lgure 18 which had the lerbet retlo of pigment to arler. Those of Flgure 18 hed feirly les ${ }^{\circ}$ incresse in drying time. 
$T e b l \oplus 16$

Lrying de to for linseed ofl contalning 1 gram lesd and 7 groms lemp bleck per 100 grems 011. (Peroent 011, 90.8)

6 months eging

it. semple, .2196 w. oll,
9 months esing

it. semple, . $1687 \mathrm{~g}$.

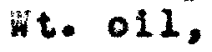

14 months ing

it. semple, .2092 g. wt. 011, .1898 g.

Time, Ineresse Inorease Inerease increese Increase incrense Hours in weight, in weight in wolght, in weight in weight, in weight grams of oll grems of oil grams of oll

$\begin{array}{lcclccc}7 & 0.0012 & 0.6 & & & 0.0004 & 0.2 \\ 8 & 0.0032 & 1.6 & & & - & - \\ 10 & 0.0087 & 4.4 & 0.0042 & 2.8 & 0.0054 & 2.8 \\ 11 & 0.0127 & 6.4 & 0.0066 & 4.3 & - & - \\ 12 & - & - & 0.0096 & 6.3 & 0.0108 & 5.8 \\ 13 & 0.0181 & 9.1 & 0.0130 & 8.5 & - & - \\ 14 & - & - & 0.0150 & 9.8 & 0.0154 & 8.1 \\ 15 & 0.0212 & 10.6 & 0.0164 & 10.7 & - & - \\ 16 & - & - & 0.0171 & 11.2 & 0.0195 & 10.3 \\ 17 & - & - & 0.0182 & 11.9 & - & - \\ 18 & 0.0234 & 11.7 & 0.0186 & 12.2 & 0.0211 & 11.1 \\ 20 & - & - & 0.0198 & 13.0 & 0.0227 & 12.0 \\ 21 & 0.0248 & 12.4 & 0.0200 & 13.1 & - & - \\ 22 & - & - & 0.0200 & 13.1 & 0.0231 & 12.2 \\ 25 & - & - & 0.0206 & 13.5 & - & - \\ 27 & - & - & -0.0 & - & - \\ 31 & 0.0260 & 13.0 & 0.0204 & 13.4 & -0243 & 12.8 \\ 38 & - & - & 0.0188 & 13.0 & - & - \\ 46 & 0.0257 & 12.8 & 0.0184 & 12.7 & - & - \\ 48 & - & - & - & - & 0.0237 & 12.5\end{array}$




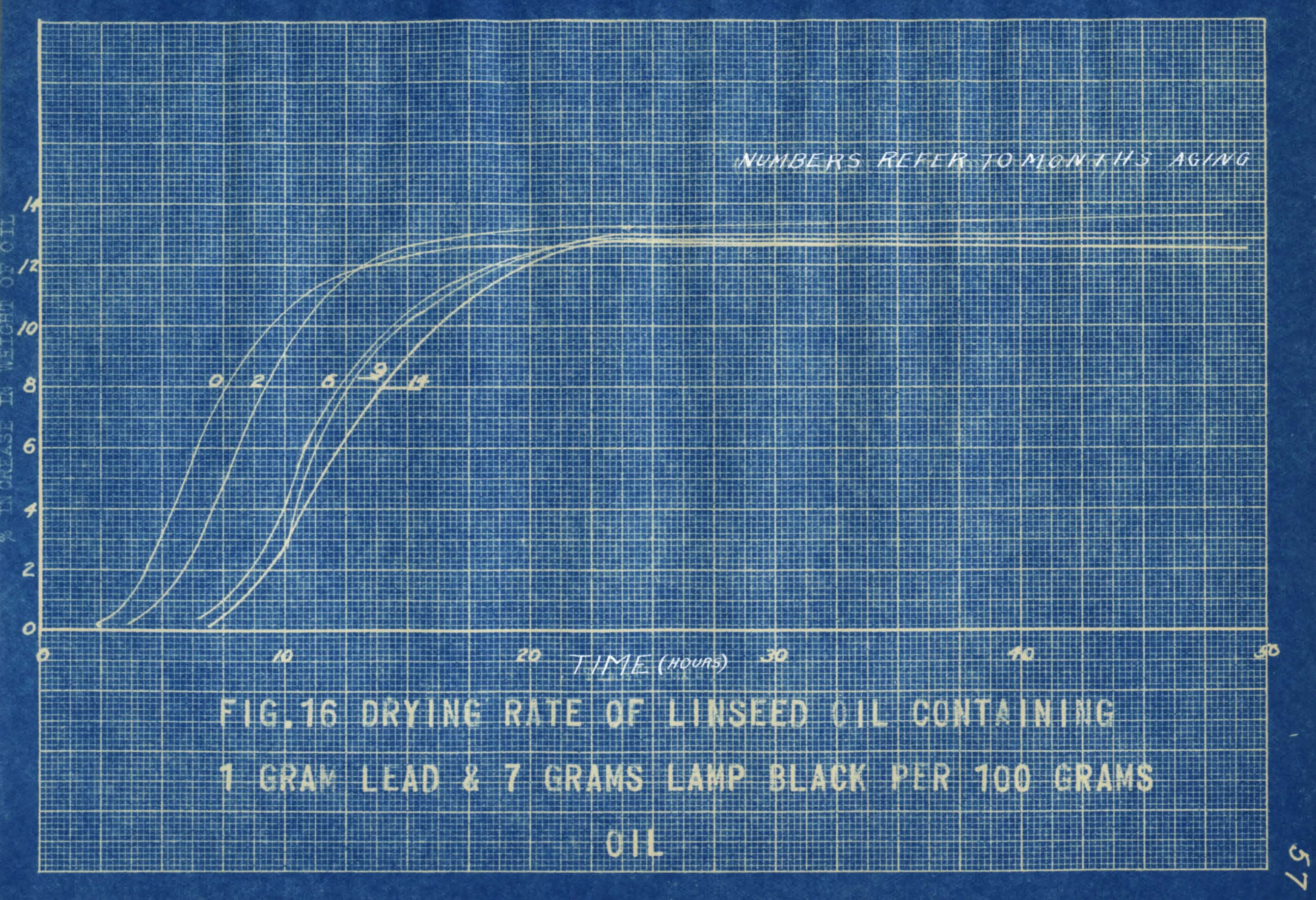


Is ble 17

Drying de to for linseed oll conteining 2 grems losd end 7 grems lemp bleck per 100 grams 011. (Peroent 011, 86.8)

6 months geing

t. semple, 2929 8. at. semple, .1683 g. it. o11.
9 months eing

ît. 011,
14 months eging

wt. gemple, $.2678 \mathrm{~g}$. t. o11, .2320 g.

Time, Incresse incresse Increese inorease Incrense increese Hourg in wight, in weight in weight, in weight in weight, in weight grams of o11 grams of oll grams of oll

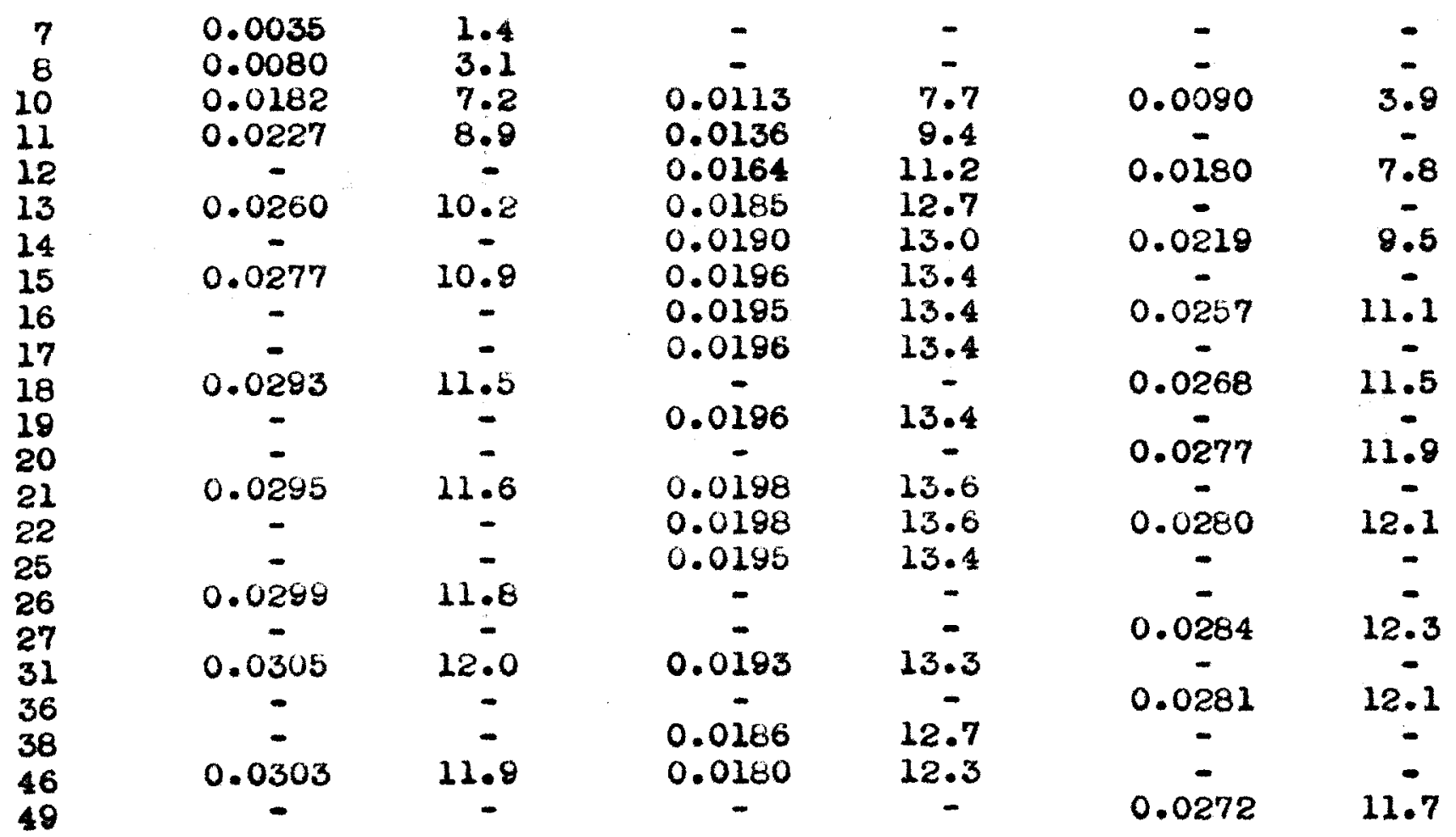


KEUPFEL A ESSER CO., N.Y. NO. 359-11



9 
Tablo 18

Drying dete for linseod oll conteining 1 gram les and 14 grems lemp bleck per 100 grams 011. (Peroent 011, 84.5)

6 months eging

it. semple, .2885 \%० nt. o11, .2522 8
9 months going

w. semplo, .1724 8 t. 011 . $.1458 \mathrm{~B}$
14 monthe egino

it. 8 smple, .2267 8 .

int. o11, .1915 g.

Time, Increase incresse Increase increase Increase increage Time, Increase incresse Incresse increase Increpse increase
Hours in welght, in welght in welght, in weight in weight, in weight grams of 011 grems of 011 grems of 011

$\begin{array}{lcc}10 & 0.0001 & 0.0 \\ 11 & 0.0009 & 0.4 \\ 12 & - & - \\ 13 & 0.0017 & 0.7 \\ 14 & - & - \\ 15 & 0.0034 & 1.3 \\ 16 & - & - \\ 18 & 0.0084 & 3.3 \\ 20 & - & - \\ 21 & 0.0156 & 6.2 \\ 22 & - & - \\ 24 & - & - \\ 25 & - & - \\ 26 & 0.0240 & 9.5 \\ 27 & - & - \\ 28 & - & - \\ 31 & 0.0284 & 11.2 \\ 32 & - & - \\ 36 & - & - \\ 38 & - & - \\ 46 & 0.0309 & 12.3 \\ 49 & - & - \\ 53 & 0.0325 & 12.9\end{array}$

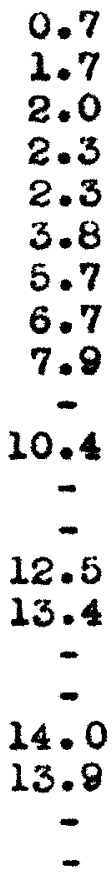

0.7

0.0024

0.0029

0.0033

0.0033

0.0056

0.0083

0.0087

0.0115

0.0152

$-$

0.0182

0.0196

0.0204

0.0202

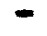

0.0008

0.0008

$-$

0.0030

0.0044

0.0090

0.0122

0.0164

-

0.0199

-

$0.0 \overline{235}$

0.0245

$-$

0.0252
$\%$ 


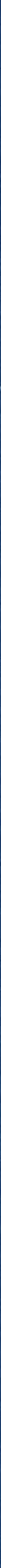


Drying de te for linseed ofl conteining 2 grems loed and 14 grems lamp blfck per 100 grams 011. (Percent 011, 81.7)

6 monthe exins

at. semplo, .2881 g. it. 011. .2435 8 .

$\%$

Time, Incresse increese Hours in welght, in wolght grems
9 months 8 ing

Wt. 8Emple, .2355 8 t. $011, .1920 \mathrm{~g}$.
14 months oging

int. semple, .2467 g. t. $011, \quad .2016$ k.

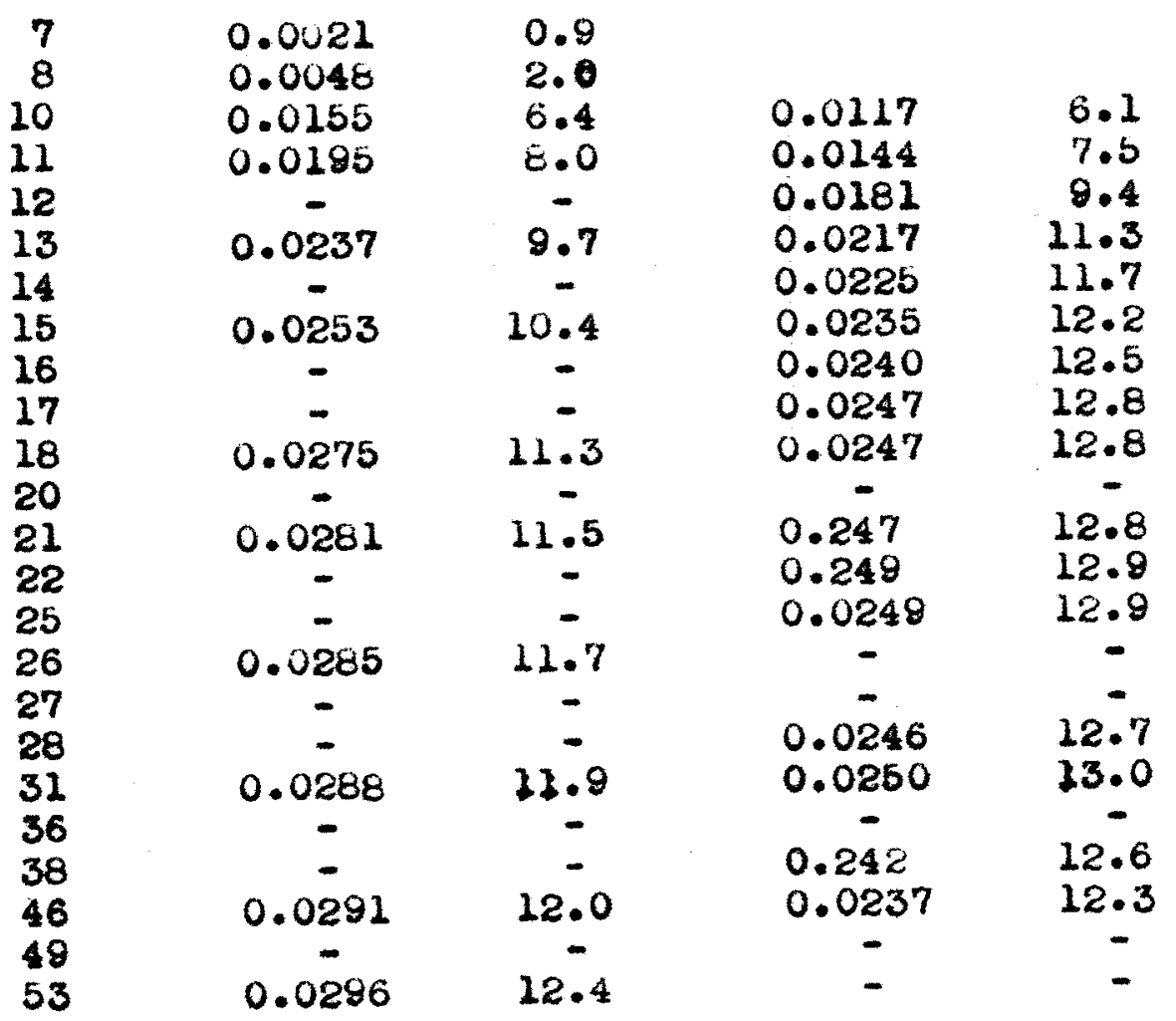

0.0060

0.0127

0.0170

0.0210

$0.0 \overline{219}$

0.0229

$0.0 \overline{231}$<smiles>C1CCCC1</smiles>

0.0239

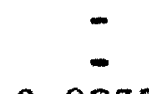

0.0233

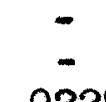

.0228

\section{$\%$} Incres 80 in weight of 011 


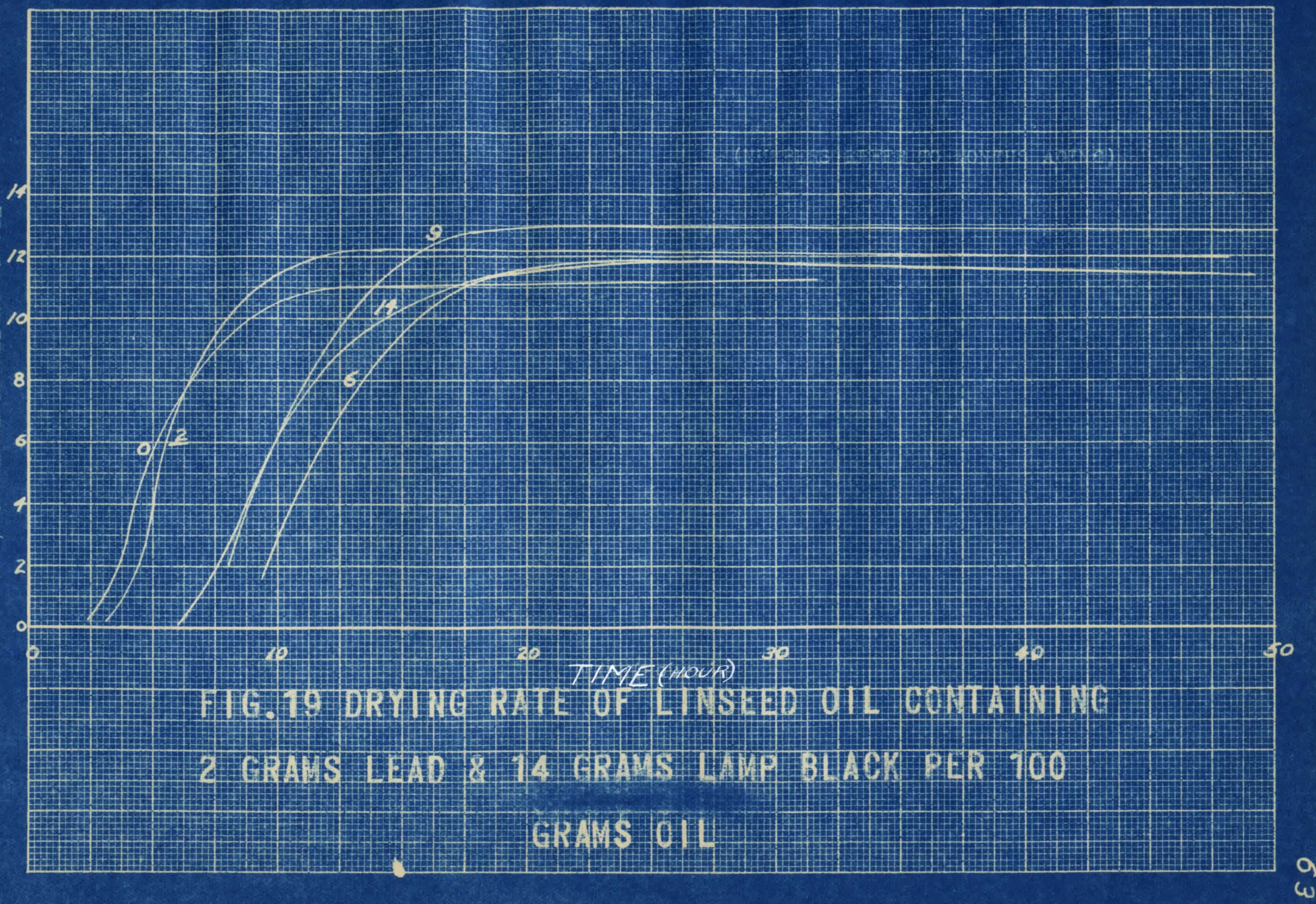


GhSPEITE 
The drylng rote curves for linseed oll conteining lefd drier and grephite were given in Figures 20,21 , 22, and 23 and were of the following composition:

Plgur. 20 -- 2 grem lod and 14 grems grephlte per 100 grems 011 .

Floure 21 -- 2 grems lezd end 14 grams graphlte per 100 grams 011.

Floure 22 -- 1 gram lesd and 28 grams grephlte per 100 grams 011.

Fl gure 23 -- 2 grems lead end 28 grams graphlte per 100 grams

In none of these fieures wes the incresse in induction

perlod greater then from two and on-holf houra (fresh

semplesi to a meximum of seven hours. Increesing the per-

cent of drier or pigment did not notloenbly chrnge the

corresponding dryling retes. 
Teble 20

Drying dete for linsed oll conteining 1 grem load and 14 froms graphite per 100 grems o11. (Percent o11, 84.7)

6 mon the eging

ut. mple, .3383 z. it. semple, .1447 w. o11.
9 months foing

at. oll
.1225
14 months oginf

Ht. semple, .2464 8. w. 011. .2085 8 .

Time, Increase increrse Inerease increase Increase increese Hours in weight, In wight in weight, in weight in welght, in weight grems of ofl grems of oll grams of oll

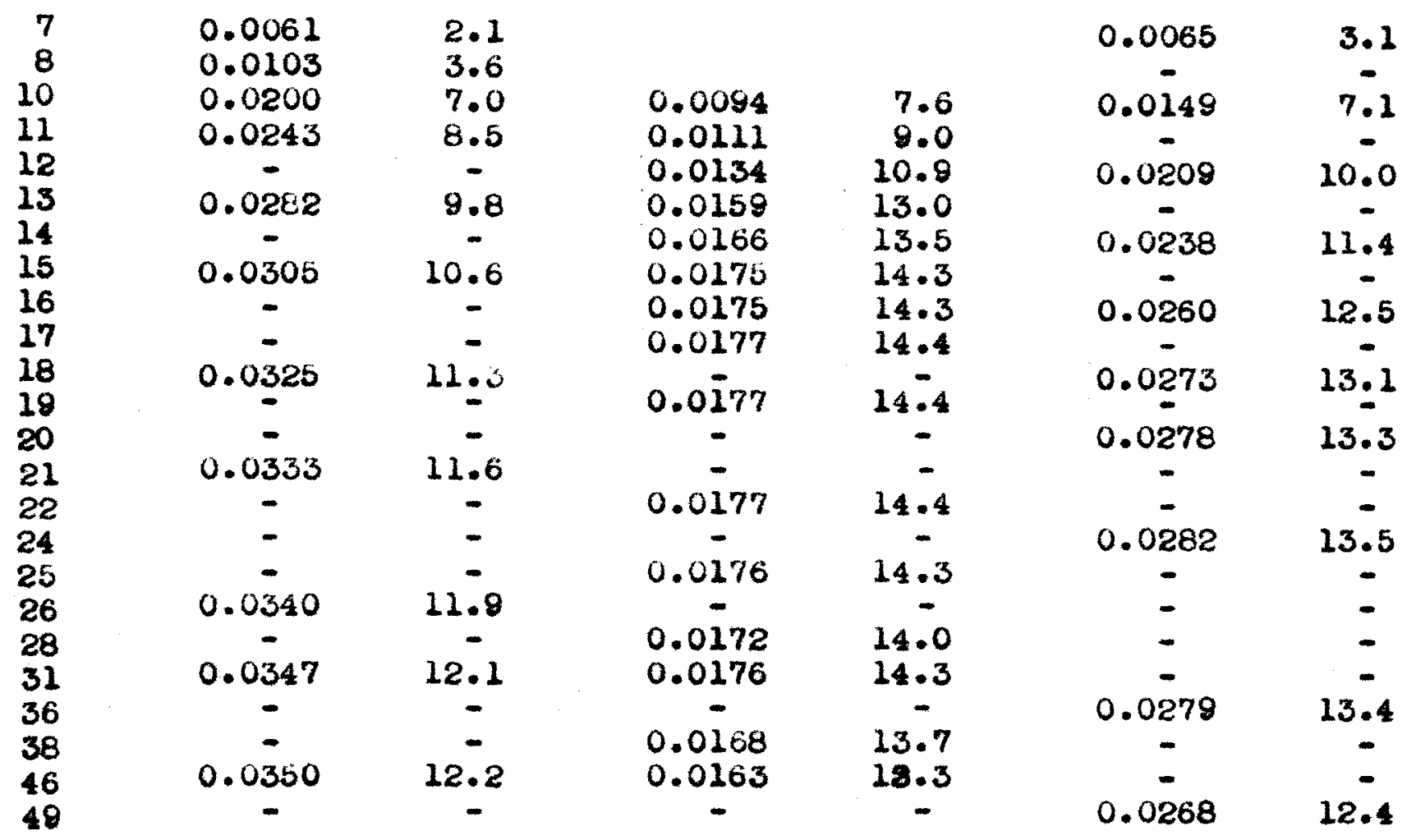




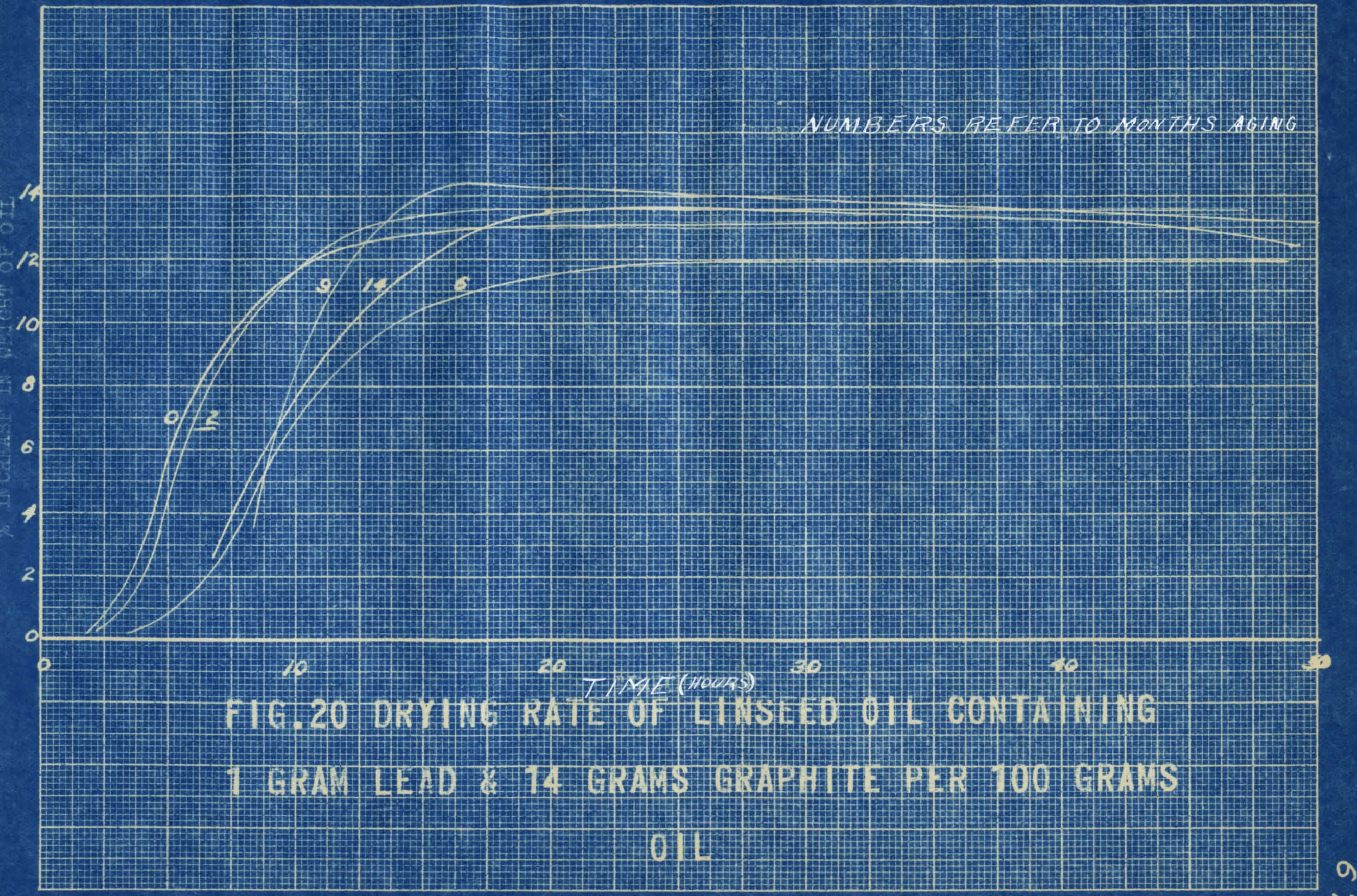


Teble 21

Drying de te for linseed oll conteining 2 grems leed and 14 grems grephite per 100 grams oll. (Percent 011, 81,9)

6 months g ging

t. semple, .3051 it. o11.
9 months going

wt. semple, .4234 8. Wt. semplo, .2904 B. it. 011, $.3463 \mathrm{~g}$.
14 months eging

nt. $011, .2380 \mathrm{~g}$.

Time, Incresse increase Inorease increase Inoresse inorease Houre in weight, in weisht in welght, in weight in welght, in we ight grams of oli grams of o11 grems of oli

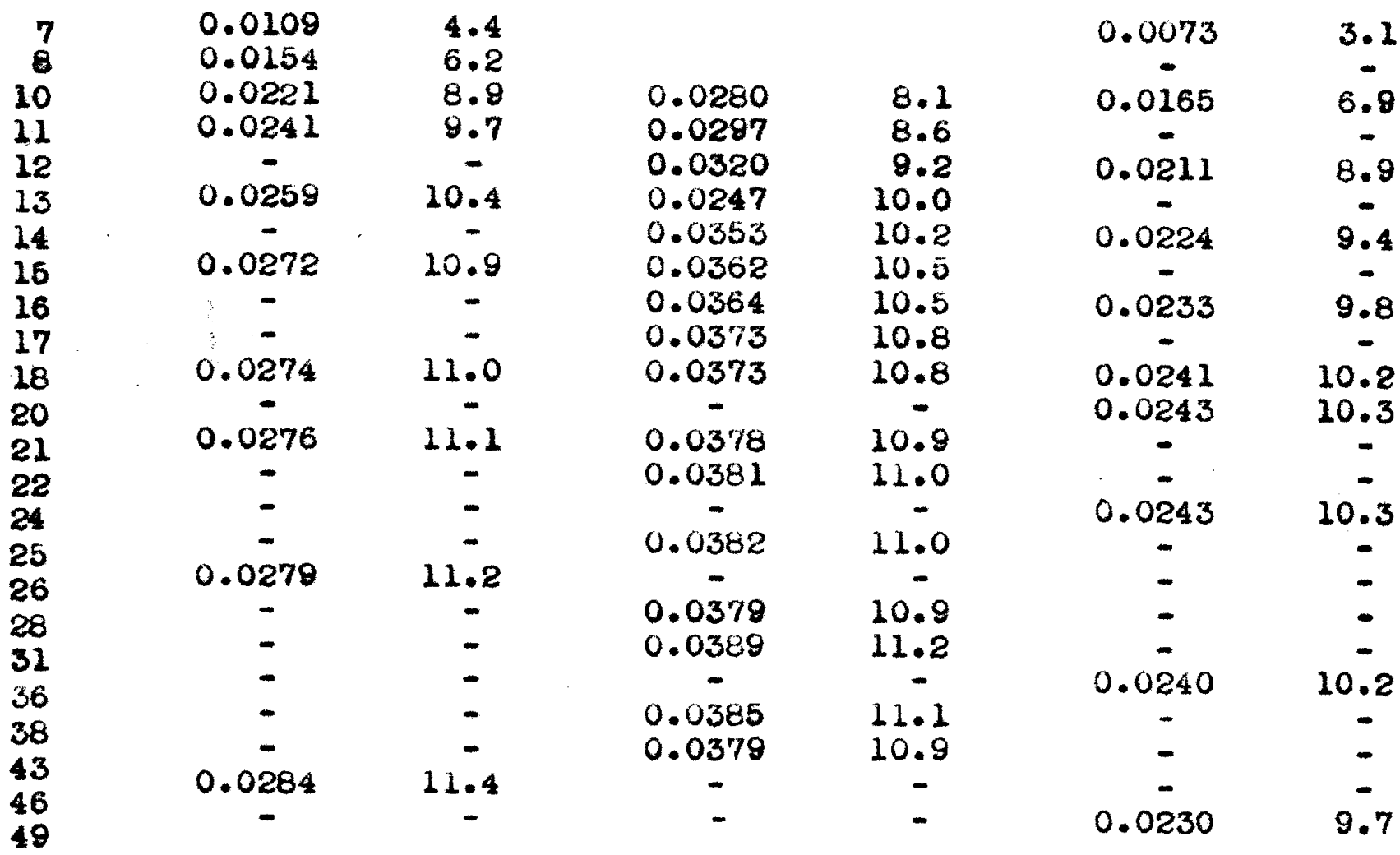


Teble 22

Lrying de te for linseed oll conteining 1 grem lerd a d 28 grems graphite per 100 grams 011. (Peroent 011, 75.7)

6 months esing

t. se mple, $.3216 \mathrm{~g}$. it. o11,
9 months Eging

wt. sample, .2877 8

it. o11, .2177 8 .
14 months eglng

t. sample, .2362 g. t. o11, .1785 $\mathrm{s}$.

Time, Increeso inorease Increese increase Increase increase Hourg in weight, In weight in welght, in weight in welght, in weight grems of oll grems of oll brems of oll

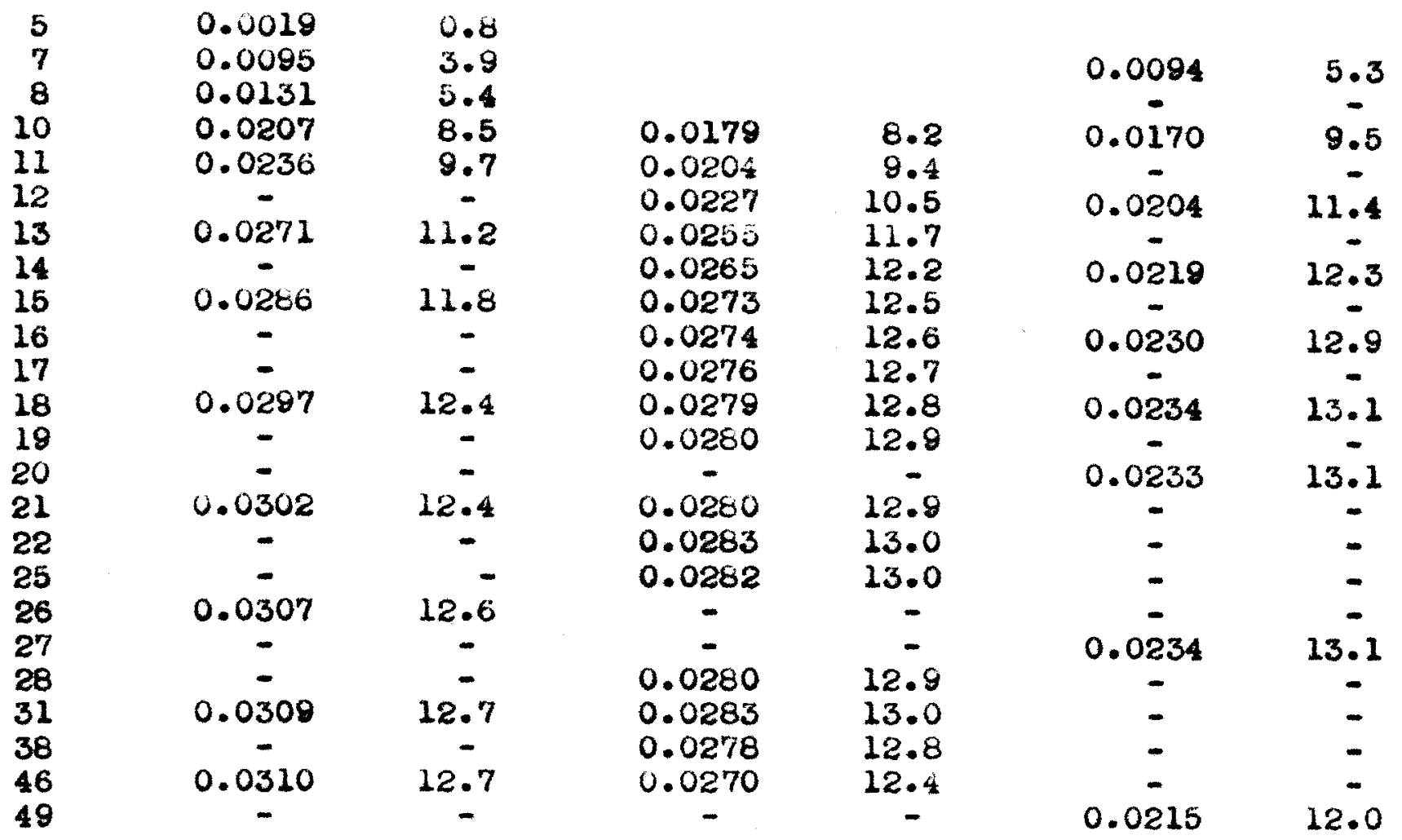




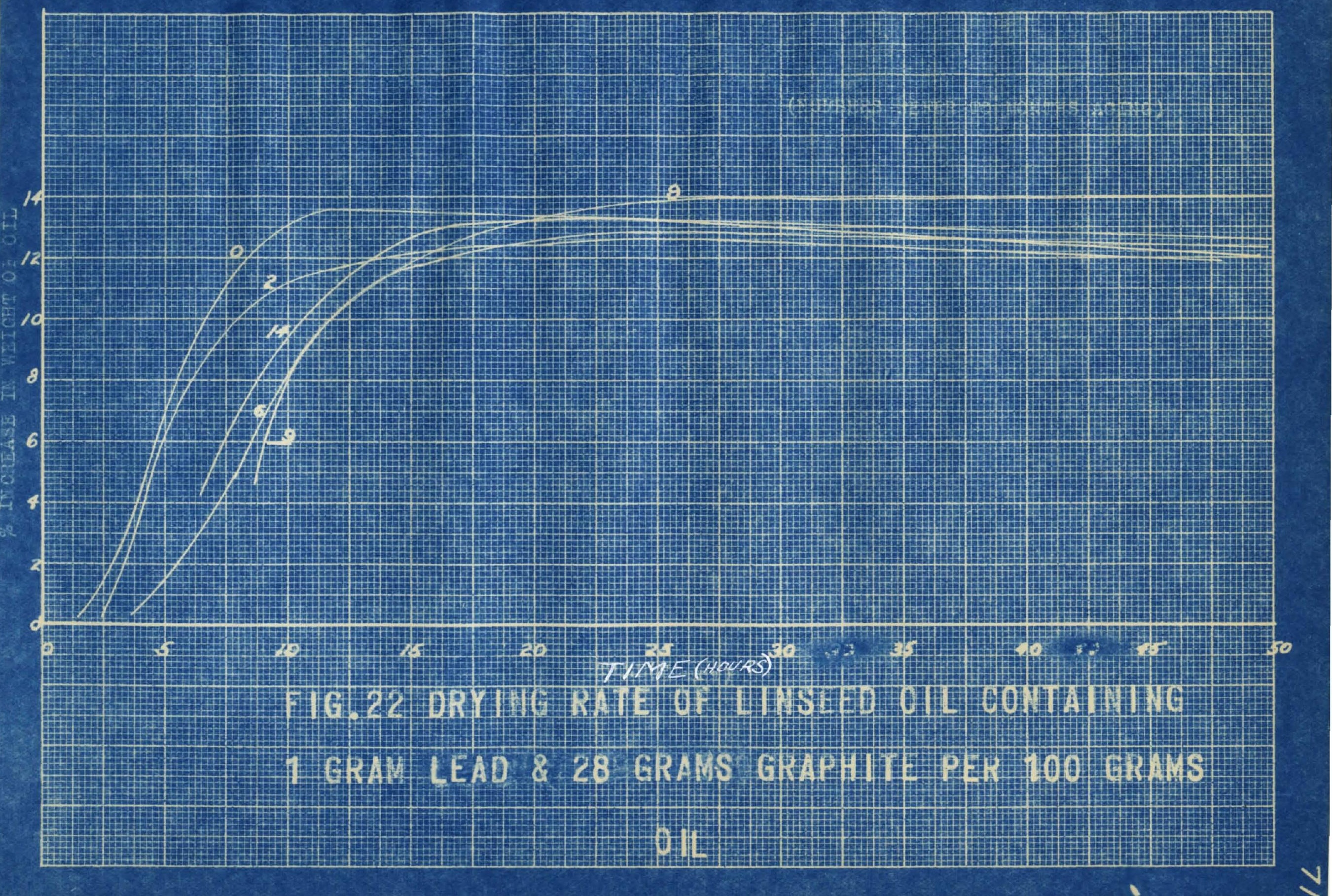




\section{Table 23}

Drylng de ta for linseed oll contalning 2 grems leed e rid 28 grams grephlte per 100 grems oll. (Peroent oll, 73.3)

6 months sing

t. semple, .3358 g. ut. semple, .2283 t. 011 . $.2482 \mathrm{~g} . \mathrm{Wt} .011$.

\section{4 months exing}

Wt. se mple. .2393

it. oll,
.16748. $.1750 \mathrm{~g}$.

Time, Incresse incresse Ineresse increrse Incresse inorease Hours in weight, in weight in weight, in weight grams of ofl of 011
In welght, in wo ight grems of oli

$\begin{array}{rcccc}5 & 0.0006 & 0.2 & & \\ 7 & 0.0127 & 5.2 & & \\ 8 & 0.0163 & 6.6 & 0.0186 & 11.0 \\ 10 & 0.0214 & 8.6 & 0.0193 & 11.5 \\ 11 & 0.0236 & 9.5 & 0.0203 & 12.1 \\ 12 & - & - & 0.0218 & 13.1 \\ 13 & 0.0244 & 9.8 & 0.0221 & 13.2 \\ 14 & - & - & 0.0226 & 13.4 \\ 16 & 0.0254 & 10.2 & 0.0224 & 13.4 \\ 16 & 0.0258 & 10.4 & 0.0221 & 13.2 \\ 18 & 0.0264 & 10.6 & 0.0218 & 13.1 \\ 21 & - & - & 0.0218 & 13.1 \\ 22 & -0.0264 & 10.6 & 0.0216 & 12.4 \\ 25 & - & - & - & - \\ 26 & - & - & - & - \\ 27 & 0.0262 & 10.5 & -212 & 12.6 \\ 28 & - & - & - & - \\ 31 & 0.0263 & 10.6 & 0.0207 & 12.4 \\ 38 & - & - & - & 12.0 \\ 46 & & - & -\end{array}$

0.0087

0.0154

0.0180

0.0187

$0.0 \overline{192}$

0.0195

$0.0 \overline{196}$

-

0.0197

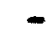

$-$

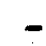

$-$

0.0284
5.0

8.8

10.3

20.7

11.0

11.1

11.7

$-$

$-$

11.3

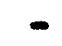

-

$-$

$-$

10.5 


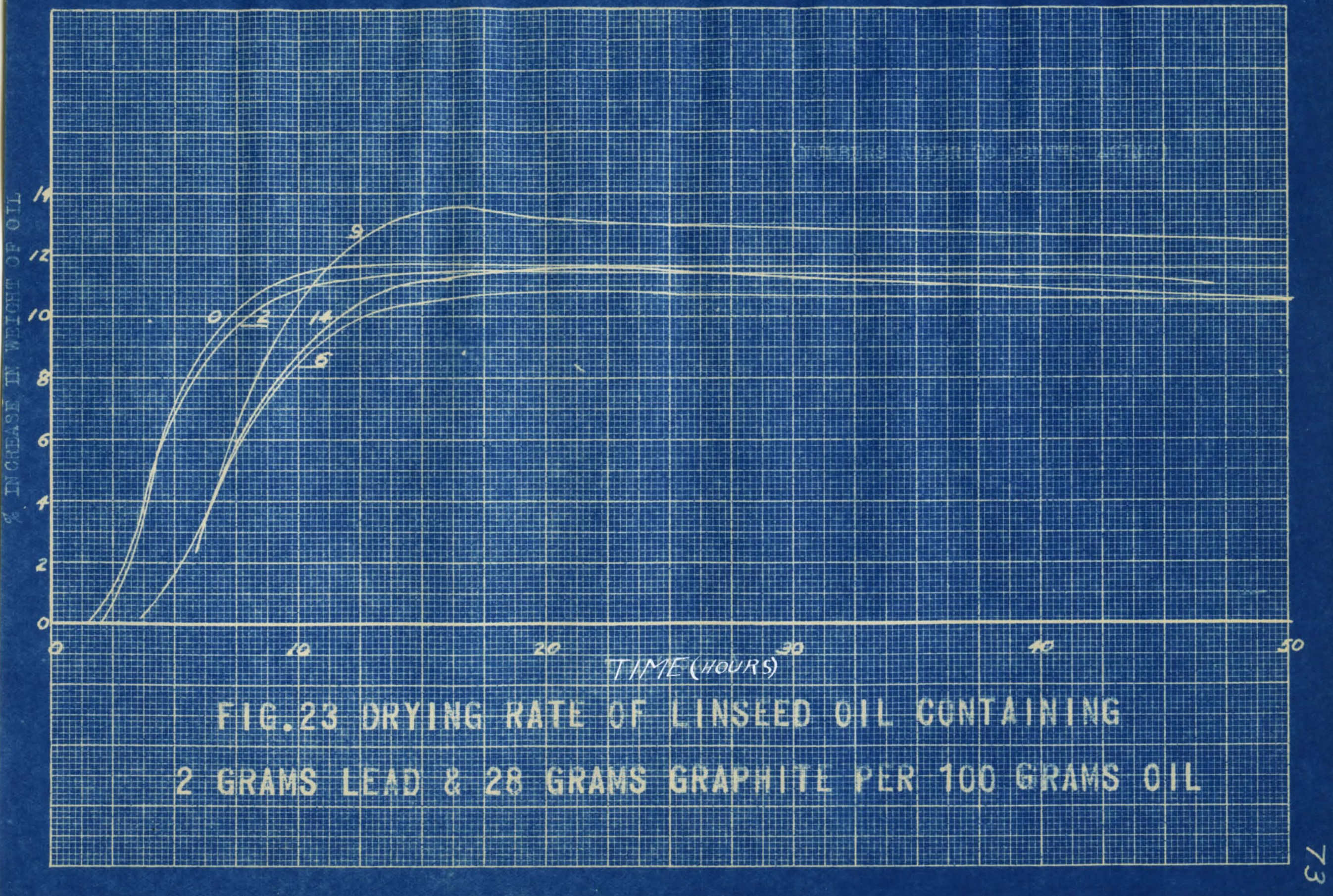


SUMMAKY 
Prom the flgures previously shown it was clearly Indiceted thet cerbon bleck ofused the greetest losses In arylng power of the linseed oll $\mathrm{mixtures}$ of wiok end drier. Bone bleck, lemp bleck, and grephite followed in order as exhibiting losses in drylng power. Bone bleck coused folriy large loss of drying ablity the small pe te of dryinge. Lemp bleck geve modere te losses in drying ablilty. The decreese of drylne wes very smell in the case of graphite.

Carbon block, ocusing the greetest losses in drying power end beine the most commonly used pligment of those Investigetes, was chosen s the plgment with which to work in the stady of methods of preventing or decreesing the loss in drying power. 
Pf KT II

EXINIHENTEL 
S. thod for decreasing or preventing the losers in arying power on eging ofrbo black peints were studied. The verlous methods of treeting the bleck re siven be low:

A. Grinalng the plgment in oll with 2 ino napthenete, 11 therge, end eluminum serrete in the expecte tion thet fllms of the soeps would form sround the plgment porticles, thus decreesing the adsorption of the drier.

B. Trefing plizment wh solution to remove adsorbed oerbon monoxide and eqroor dioxide which mey be possible sources of the losses of drying bility. The regrinding of certin n ged smplos wes investigeted. Compost tions of the se semples ere given in table I. 
The mixture of Flgure 24 contelns .1 gram eluminum stefre te, .04 grams cob-1t, and 3.5 grams ofrbon bleck per 100 grems 011. The steere te wes ground with the plgment In tine oll end the arier we odded with fdditionel grinding. Three wonths oling showed en Increese in Induction period from two to far and one-helf hours. Ihls is e very smell Inores se compered to rlgure 10 which contelne the seme emount of cobelt end cerbon block. The in rese in induetion perlods for two monthe of Flgure 10 is from two and one-half to Afteen hours.

Ploure 25 exhlbits arying re tes of semples conteining .1 grem kino, .04 gram cobe 1t, end 3.5 grems cerbon block per $100 \mathrm{grwms} 011$. The 21 no, s suodex, wa ground with the oleck in oll and oobelt arler ef edded s in the previous orse. The ine reese in induction periods for the sample which hed eges tirree months wo from two to four

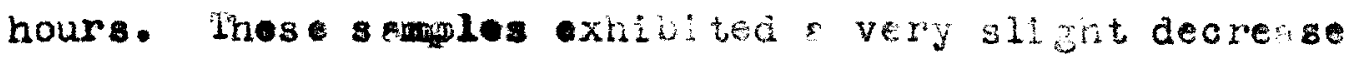
In $108 s$ of drylag powtr es compered to the samples of rigure 24

In lgure $26(.1 \mathrm{grem} 21 \mathrm{no}, 1$ gren lend, end 3.5 grams cerpon bleck per 100 grems oll) the formuletion of the semple we latentloel to those of figures 24 nd 25 . An Induction period inorease from four hours (fresh semple) to e1ght hours (three month's semple) wo shown. These perlode wore considerebly greater than ei ther of the two previous semples witch ha cobelt for the arier. Figure 2 , 
b. vine the seme smount of leed drler end cerbon blfck displeyed only slightly greeter lncreese for the first two montis.

To study the effect of adsorsed geses on the plgment perticles, the certion bleck wrs treeted with sclutions of Emmoniacel cuprous onloride and potassum hydroxide of the seme conoentretion sis those used in ges enelysis work for absorbing cerbon monoxide nd cerbon dioxide respectively.

for treetment to remove cerbon monoxide the plement wes kept in costact aith ouprou chloride snd given intermittent grinding. The pigrent wes then we shed thoroughlJ, dried, end mede into semple containlug $.04 \mathrm{grem}$ cobelt and 3.5 grems cerbon bleck per 100 grams o1d. Drying curves of this mixture re shown in 1 gure 27 . The induetion perlod incrase is from tho and one-helf hours (fresh sm plo) to seven hours (tiro month's smple). The untrested Homent inixture of the semple composition, figure 2, displayed Induction periods for the fresh semple of two nd one-helf hours, na for the two month's semple, fifteen hours. This indiested thet the treetment for removel of es rbon monoxlate very definltely decreesed the loss of drying power.

Treetment for removsl of scroon dloxide wes performed In the seme menner es for the tweetment for reoving the cerbon monoxide. Trenty-flue percent petessium hydroxido 
w. used for the trestment of the ombon bleck. Figure 28 exhlbits dryling retes of this mixture which wes of the seme composition es thet of slgure 27. For the fresh semple the Induction period we two end one-helf houra end for the two month's semple flve hours os compered with two nd fifteen hours of the semple mixture with untref ted blook, Pgure I. A sample heving the compositior es the two previous semples but with plement treeted with $5 \%$ solution of potesslum hydroxide, hes arylng ourves in Flgure 29. One month' a lag showed en inoresse from one to two hours in the irduction periods s compered to the incresse of two and one-holf to fourteen hours for one month's semple taken from bennett (1).

P1gure 30 (.25 grum le ed e lithorg, .04 grem cobelt, end 3.5 grems e robon black per 100 grems o11) end Plgure 31 (.5 gran 1 ad a 11 thorge, .04 grem cobe 1 t, end 3.5 grams ceroon bleck ber 100 grems o11) dispiejed ourves of semples In wion il therge we ground previous to the addition of arler. Both had pproximately the seme ineregse in Induotion perlod one to two hours for one month' a ging. lear old semples of rigures 9 nd 10 mere reground and the drylng curves both receded grertig. In Figure 9 the drylng curve wes epproxine tely thet of the freshly mede semple while in Flgure 10 the Induction period wes elght hours es compred to fourteen hours for the two month's sample. 
Teble 24

Drying de te for lingeed oll containing 1 grem pluminum steereto, .04 gram cobelt, and 3.5 grams aerbon black per 100 grams oll. (Peroent o11, 96.0)

Freshly mede semple

*t. 8emple, . $1256 \mathrm{~g}$.

it. o11, .1205 g.

Time,

Hours

$\begin{array}{lcc}1 & 0.0003 & 0.2 \\ 2 & 0.0003 & 0.2 \\ 3 & 0.0069 & 5.7 \\ 4 & 0.0131 & 10.9 \\ 5 & 0.0151 & 12.5 \\ 6 & 0.0155 & 12.9 \\ 7 & 0.0156 & 12.9 \\ 8 & 0.0158 & 13.1 \\ 9 & - & - \\ 10 & - & - \\ 11 & 0.0158 & 13.1 \\ 12 & - & - \\ 13 & 0.0154 & 12.8 \\ 14 & - & - \\ 18 & - & - \\ 16 & 0.0149 & 12.4 \\ 20 & & \\ 22 & & \\ 28 & & \\ 34 & & \end{array}$

1 months eging

it. semple, $.2109 \mathrm{~g} \cdot$ it. 011. .2025 g.

Ineresse increese
in weight, in welght
grems

$\begin{array}{cc}0.0007 & 0.3 \\ 0.0082 & 4.0 \\ 0.0170 & 8.4 \\ 0.0208 & 10.3 \\ 0.0224 & 11.0 \\ 0.0229 & 11.3 \\ 0.0238 & 11.8 \\ 0.0234 & 11.6 \\ 0.0233 & 11.6 \\ - & - \\ - & - \\ - & - \\ 0.0233 & - \\ - & 11.6 \\ 0.0230 & - \\ 0.0229 & 11.4 \\ 0.0230 & 11.3 \\ & 11.4\end{array}$


Teble 24-A

Drying de te for Ilnseed of 1 contelning i grem aluminum steare to, .04 gram cobalt, and 3.5 grems eerbon bleok per 100 grams 011. (Percent 011, 96.0)

2 months oging

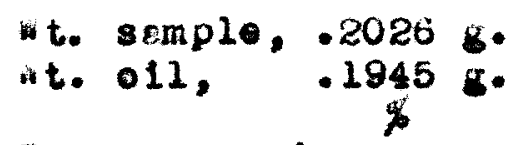

Time,

Hours

2
3
4
5
6
7
8
9
10
11
12
13
14
15
16
20
22
28
34
49
Increese increase
in wel ght, in welght grams of oll
3 months oglny

itit. semple, 1888 g.

it. 011, .1811 g.

Increese incregse

in weight, in weight grems of 011

$\begin{array}{cc}0.0002 & 0.1 \\ 0.0028 & 1.5 \\ 0.0109 & 6.0 \\ 0.0172 & 9.5 \\ 0.0198 & 10.9 \\ 0.0213 & 11.8 \\ 0.0217 & 12.0 \\ 0.0222 & 12.3 \\ 0.0223 & 12.3 \\ 0.0224 & 12.4 \\ - & - \\ - & - \\ 0.0225 & 12.4 \\ - & - \\ 0.0215 & 11.9\end{array}$




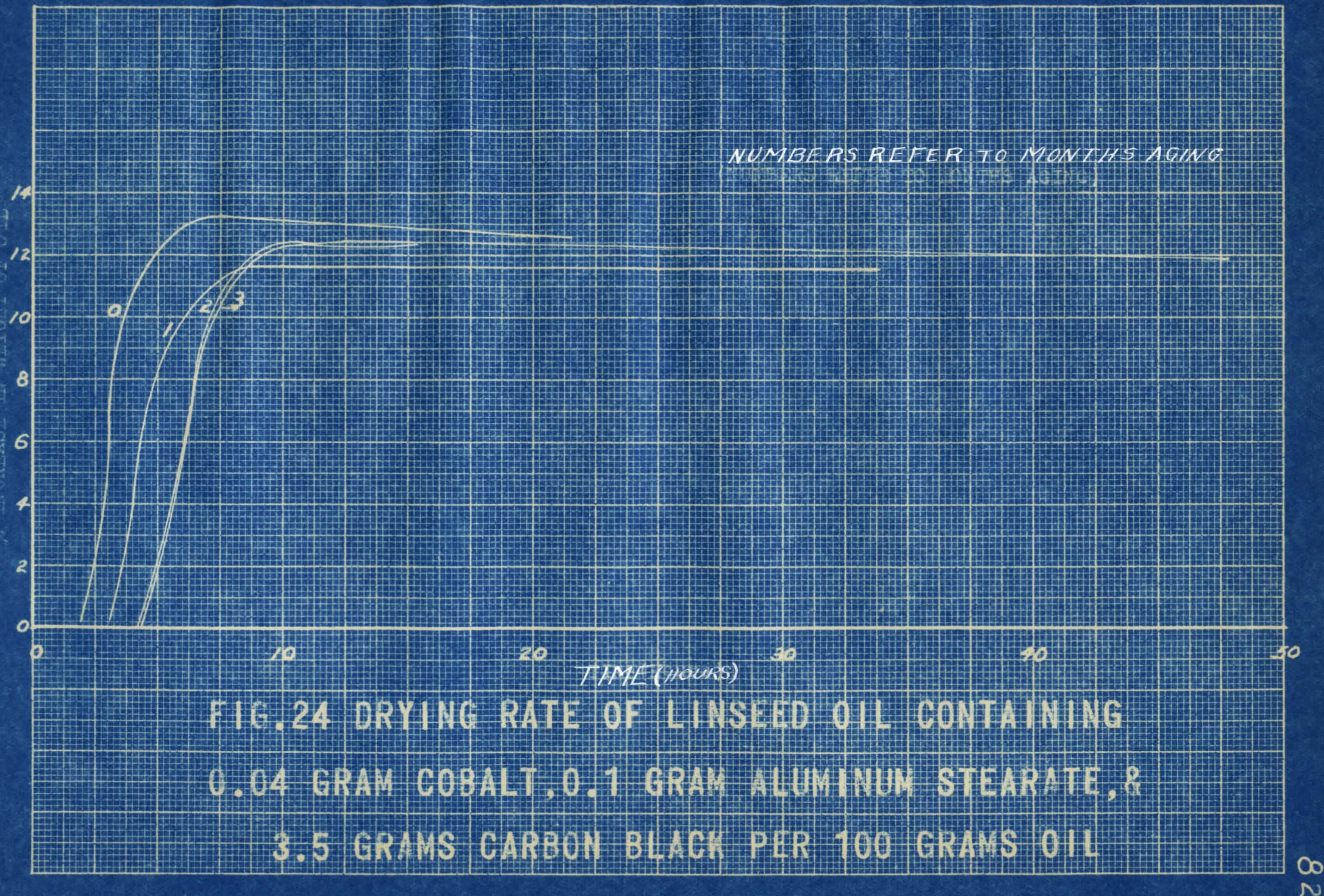


Teble 25

Drying dete for linseed ol 1 containing 1 grem zinc, .04 grem cobelt, and 3.5 grems ofrbon bleck per 100 frems 011. (Percent 011, 92.0)

Freshly mede semple

it. semple, $.1435 \mathrm{~g}$.

it. 011 . $.1320 \mathrm{~s}$.

Tims,

Hour \&

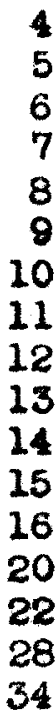

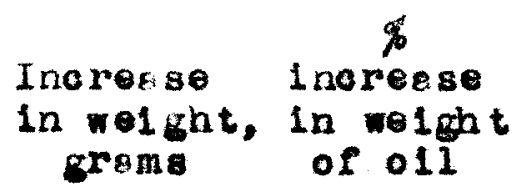

$$
0.0004
$$

0.0022

0.0067

0.0092

0.0107

0.2

1.7

5.1

7.0

8.1

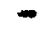

0.0160

$-$

$-$

$-$

0.0172

12.

13.0

-

-

$0.0 \overline{184}$

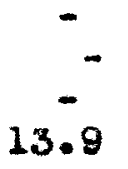

1 months oging

Ht. semple, $.1880 \mathrm{~g}$.

wt. ofl, $.1729 \mathrm{~s}$.

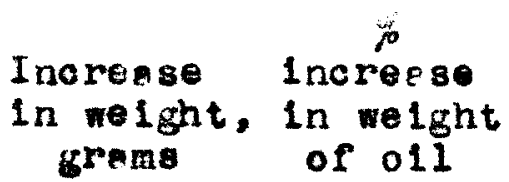

$\begin{array}{cc}0.0001 & 0.0 \\ 0.0008 & 0.5 \\ 0.0011 & 0.6 \\ 0.0029 & 1.7 \\ 0.0062 & 3.6 \\ 0.0113 & 6.5 \\ 0.0137 & 7.9 \\ 0.0168 & 9.7 \\ - & - \\ - & - \\ - & - \\ 0.0228 & - \\ - & 13.2 \\ 0.0233 & - \\ 0.0237 & 13.5 \\ 0.0235 & 13.7 \\ & 13.6\end{array}$


$1 a b 1025-A$

Lrying de te for linseed oil conteining.1 grem zinc, 04 grem cobelt, snd 3.5 grems eerbon bleck per 100 grems 011. (Percent 011, 92.0)

Ime, Hours

6

7

8

9

10

11

12

13

14

15

26

20

22

28

34

48
2 months es Ins

at. omple, $.1460 \mathrm{~g}$.

it. o11. .13428 .

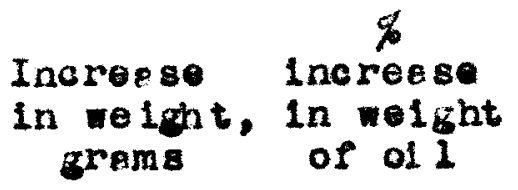

0.0001

0.0014

0.0047

0.0081

0.0134

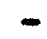

-

$0 . \overline{0183}$
3 months $A$ ging

H. senp10, $.1963 \mathrm{~g}$.

at. ol1, .18058 .

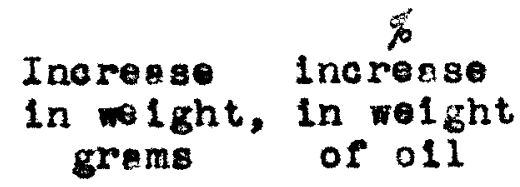

$\begin{array}{cc}0.0002 & 0.1 \\ 0.0017 & 0.8 \\ 0.0038 & 2.2 \\ 0.0080 & 4.4 \\ 0.0117 & 6.5 \\ 0.0150 & 8.3 \\ 0.0181 & 10.0 \\ 0.0205 & 11.4 \\ - & - \\ - & - \\ - & - \\ 0.0265 & 14.7 \\ - & - \\ - & - \\ 0.0258 & 14.3\end{array}$




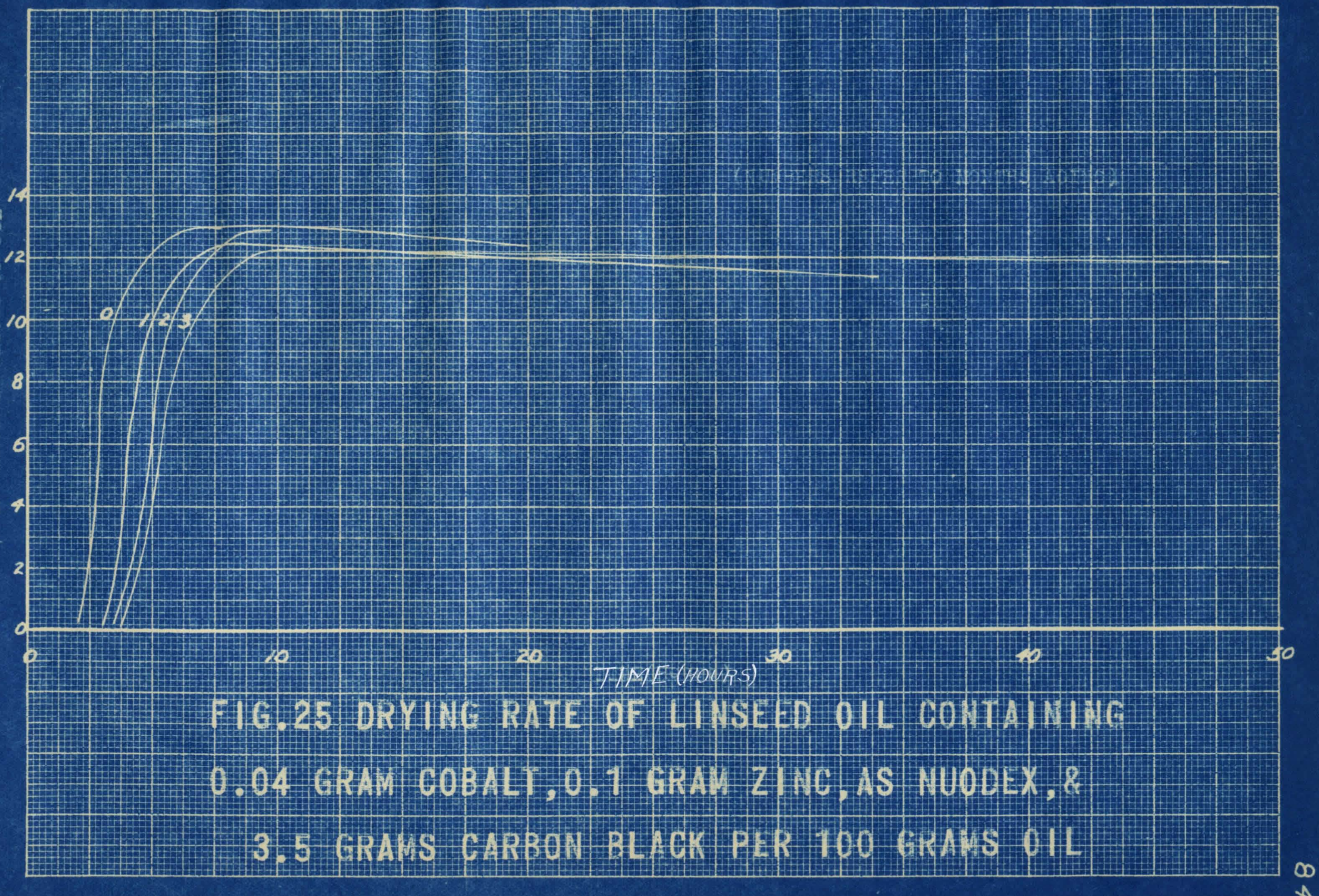


Teble 26

Drylng dete for 1 inseed oll conteinlug 1 grem zlnc, 1 grom lead, and 3.5 grems eerbon black per 100 grams 011. (Peroent o11,94.9)

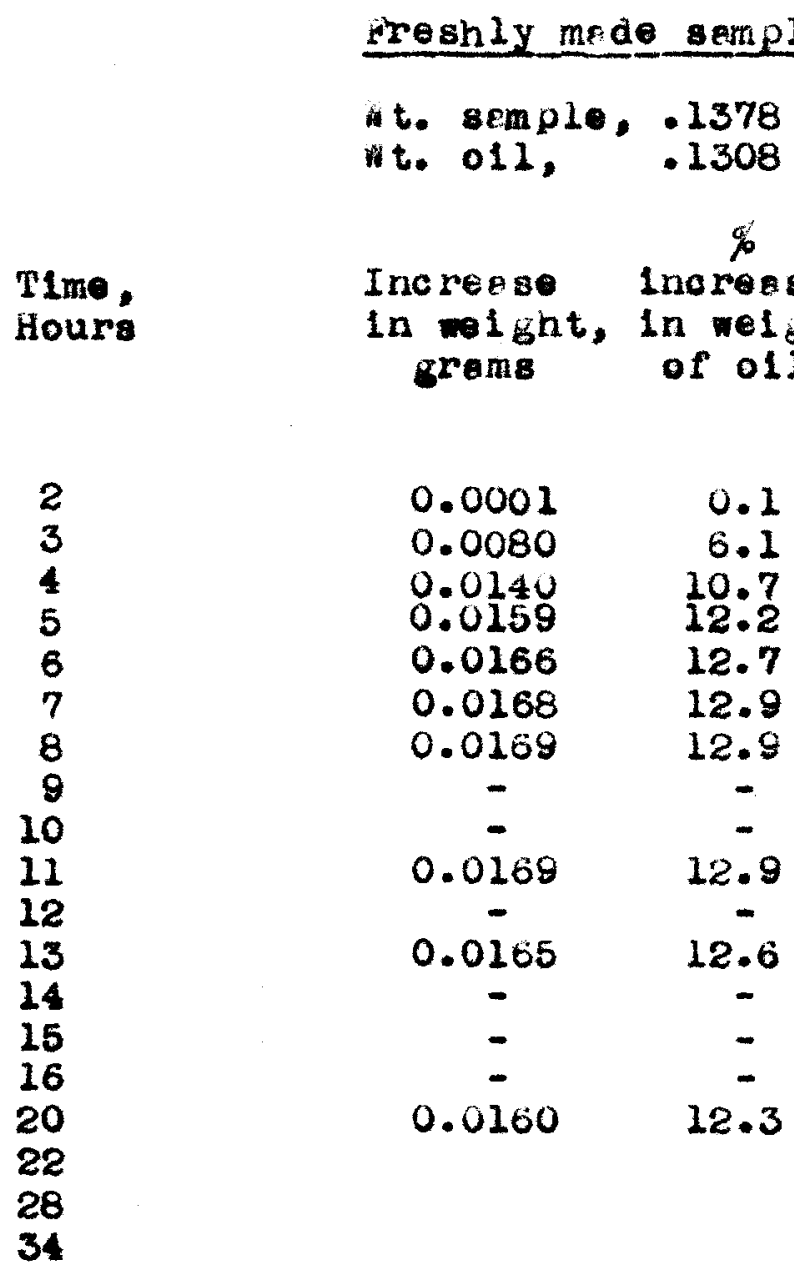

1 months eging

i. sample, . $1680 \mathrm{~g}$.

at. o11. .1593 s.

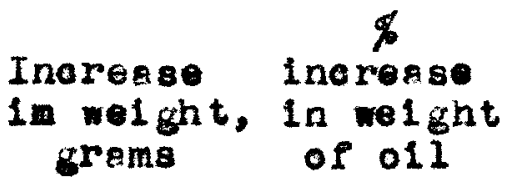

$\begin{array}{cc}0.0006 & 0.4 \\ 0.0086 & 5.4 \\ 0.0156 & 9.8 \\ 0.0182 & 11.4 \\ 0.0194 & 12.2 \\ 0.0195 & 12.3 \\ 0.0189 & 12.5 \\ 0.0184 & 12.2 \\ 0.0195 & 12.3 \\ - & - \\ - & - \\ - & - \\ 0.0187 & 11.7 \\ - & - \\ 0.0182 & 11.4 \\ 0.0180 & 11.3 \\ 0.0180 & 11.3\end{array}$




\section{Teble 26-A}

Drying date for linseed ofl containing il gram zi ne, I grem lesd, and 3.5 grems cerbon bleck per 100 urems o1l. (Percent 011,94.9)

Time, Hours

$$
2
$$

3

4

5

6

7

8

0

10

11

12

13

14

15

16

20

22

28

34

48

51
3 months exins

it. gemple, $.1934 \mathrm{~g}$. it. oll, $.1835 \mathrm{~g}$.

it. semple, $.1691 \mathrm{8}$
t. 011,

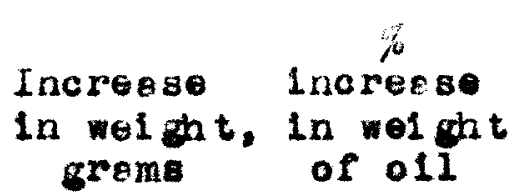

$\begin{array}{lr}0.0007 & 0.4 \\ 0.0080 & 4.4 \\ 0.0158 & 8.6 \\ 0.0200 & 10.8 \\ 0.0213 & 11.6 \\ 0.0220 & 12.0 \\ 0.0222 & 12.1\end{array}$

$0.0204 \quad 12.7$

$0.0006 \quad 0.4$

$0.0005 \quad 0.3$

0.004122 .5

$0.0138 \quad 8.6$

$0.0184 \quad 11.4$

$0.0196 \quad 12.2$

$0.0206 \quad 12.9$

$0.0205 \quad 12.8$

$\begin{array}{cc}\overline{-} & \overline{-} \\ 0.0201 & 12.5\end{array}$

Incresse increese

in wolght, in wight grems of ofl
$0.0222 \quad 12.1$

$0.0221 \quad 12.1$

$0.0226 \quad 12.3$

$-$

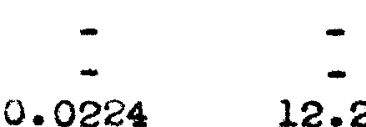

0.0218

$-$

$-$

11.9 


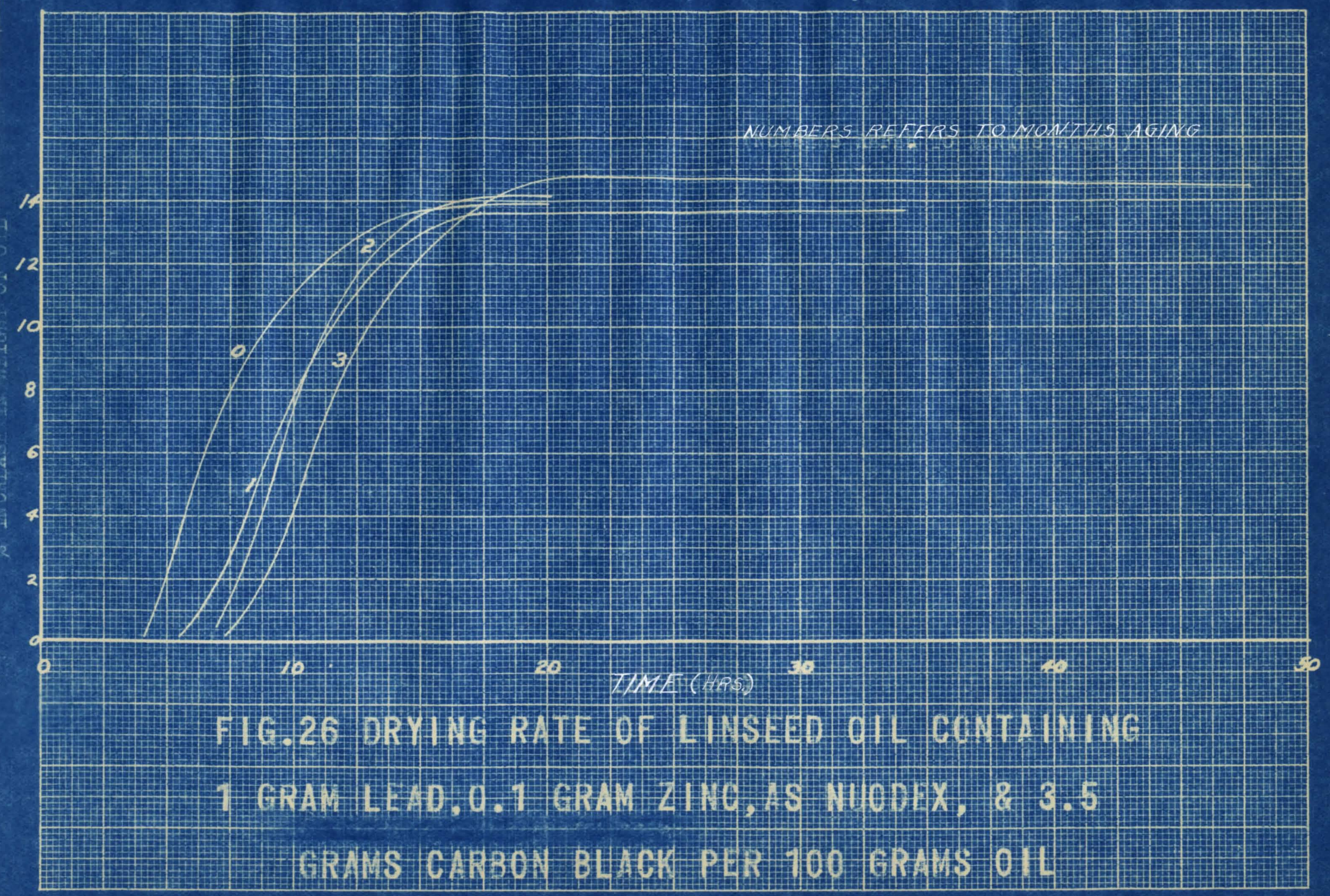

$\infty$
0 
l'eble 27

Drying dete for linseed oll caitaining .04 grem cobelt snd 3.5 grems cerbon bleck, trested ith smmonlacel cuprous chloride. (Percent o11, 96.0)

Freshly mode semple 1 months exing

it. semple, .2019 g. wt. semple, .1615 g. wt. semple, .2210 g. wt. oll. .1938 . wt. o11,
2 months ging

Ht. $\operatorname{senple,~} 2120 \mathrm{~g}$.

Time, Inoreese increase Inoreese indrease Inoregse incresse Hours in weight, in welght in wolght, in weight in welght, in weight grems of oli grems of oli grems of oli

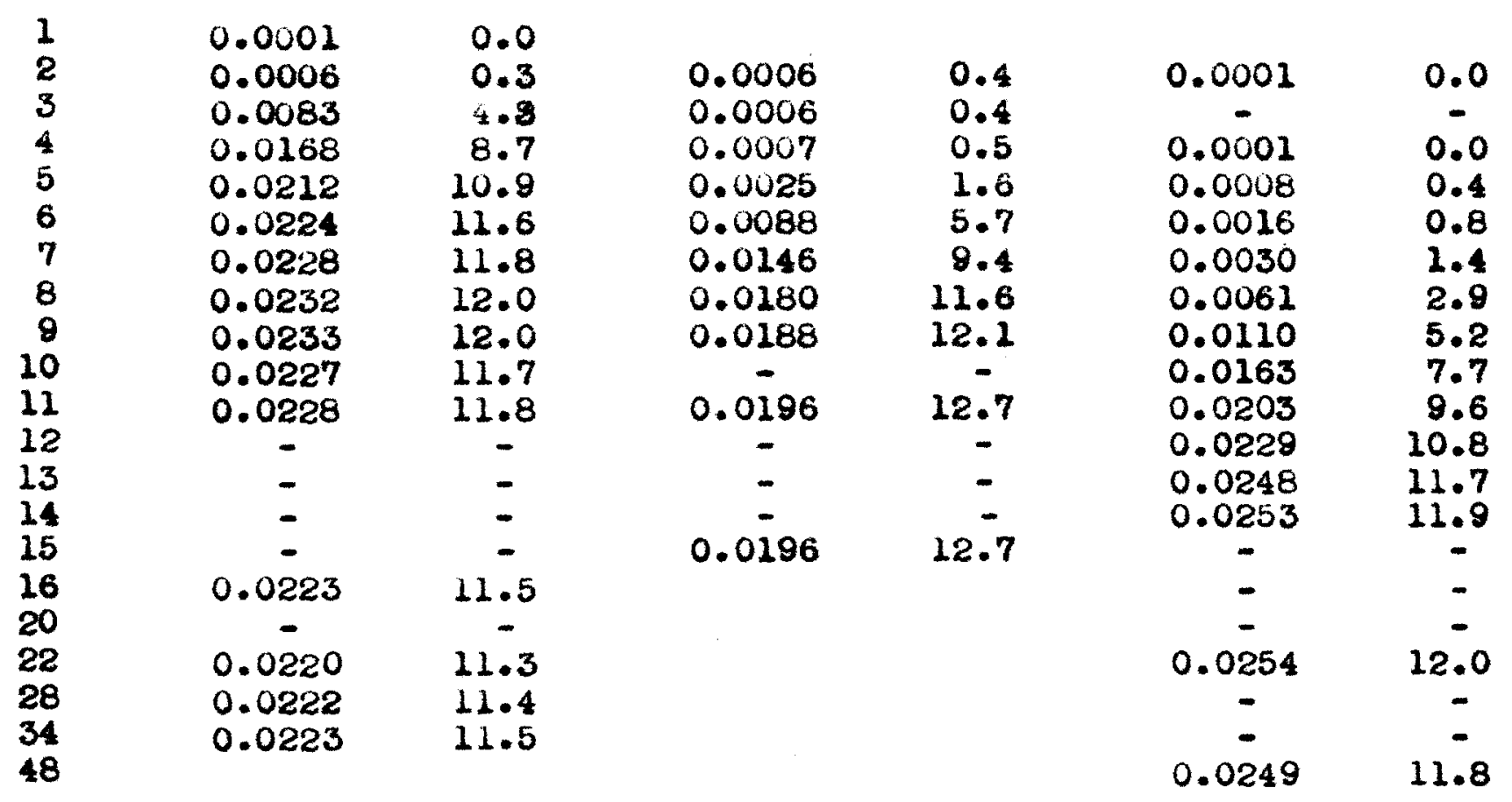




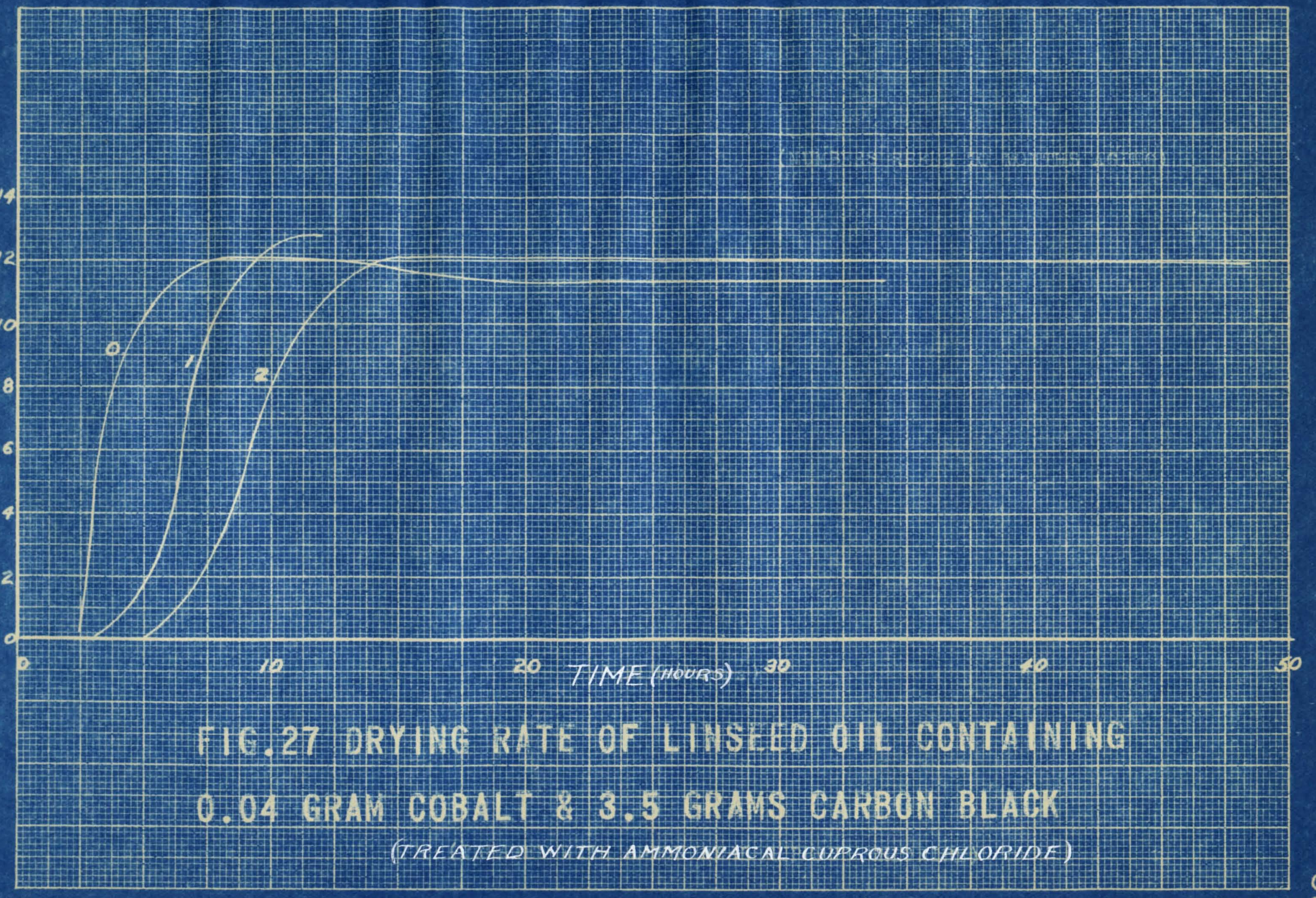


Drylng de te for 11 riseed oll contelniag .04 grem cobalt end 3.5 grems cerbon bleck, tres ted wi th $25 \%$ pots ssium hydroxide. (Pereent 011, 96.0)

Preshly mede semple 1 months exing

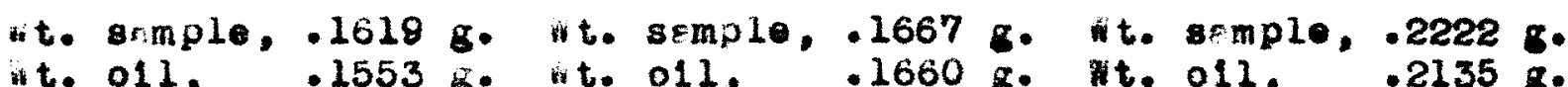

\% $\%$ \% Hours in weight, in weight in weight, in wolght in wight, in weight grems of oll srams of oll orems of 011

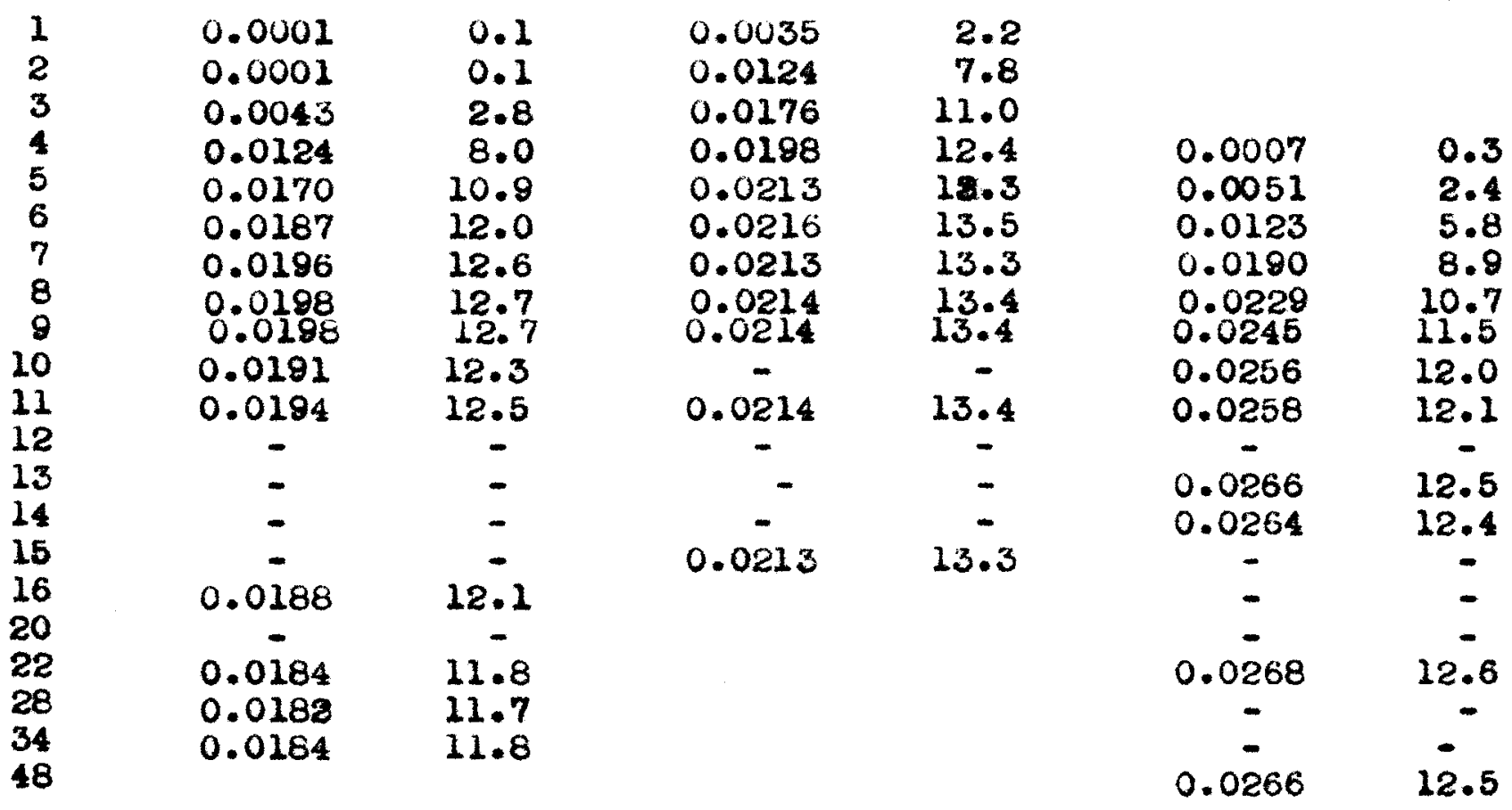



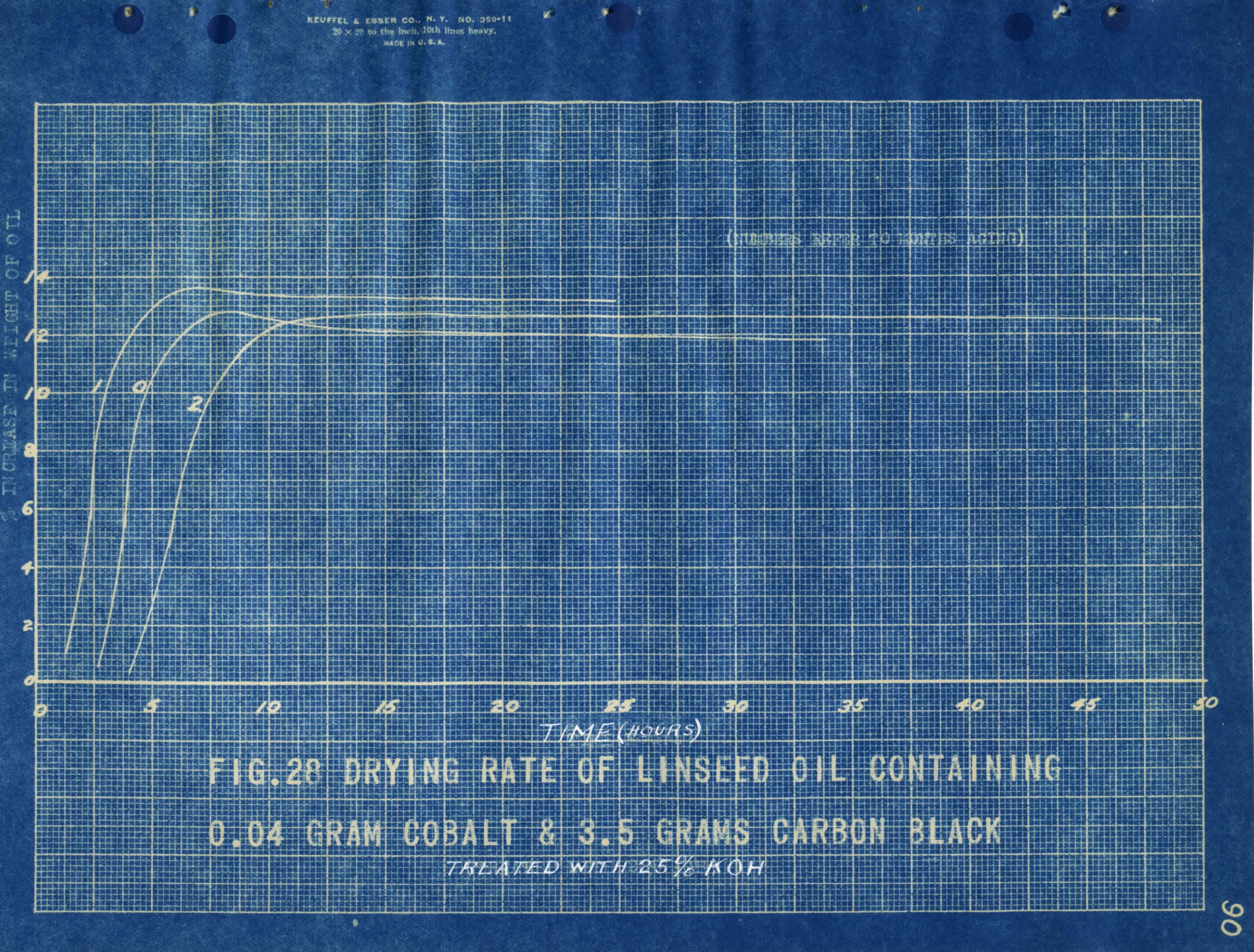
Table 29

Drying dete for lirseed oll conteining .04 grem cobelt end 3.5 grems cerbon bleok, tree ted if th $5 \%$ potessium hydroxide. (Percent 011, 96.0)

I'imo. Hours

1
2
3
4
5
6
7
9
10
13
14
21
22
38
62
Preshly made semple

"t. semplo, .2222 6 .

it. o11. .21368.

$\%$ Inarese Increase

in welght, In we ight grems of o11

0.0097

0.0171

0.0211

0.0253

0.0258

0.0258

0.0259

0.0265

$0.0 \overline{255}$

4.5

0.9

$0.0 \overline{245}$

11.8

12.1

12.1

12.2
8.0

1 monthg eging

it. semple. $.1839 \mathrm{~g}$

it. $011, .2765 \mathrm{~g}$

Increase $\stackrel{\&}{\not}$

In welght, in weight erems of o1l

0.0001

0.0016

0.0100

0.0170

0.0202

0.0

0.9

5.5

$\begin{array}{cc}0.0225 & 12.5 \\ - & - \\ 0.0231 & 12.8 \\ - & - \\ 0.0228 & 12.6 \\ -\overline{0} & -- \\ 0.027 & 12.6 \\ 0.0217 & 12.0\end{array}$




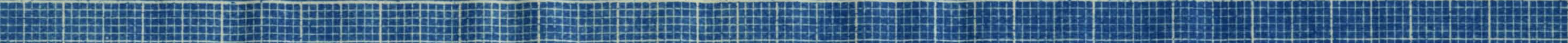

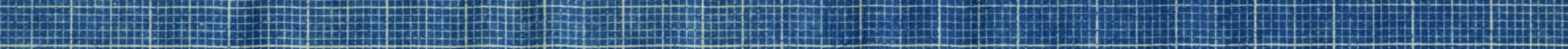
T:

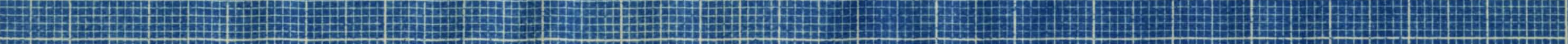

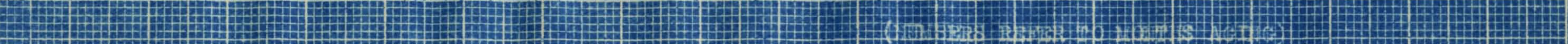

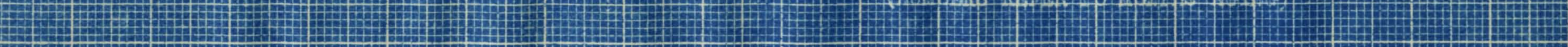

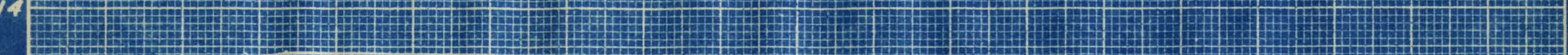
| 
Toble 30

Drying de te for linseed oll conteining .25 grem leed, a 11 therge, .04 grem cobelt, and 3.5 grams cerbon bleck. (Peroent 011, 95.8)

Time, Hours
Freshly mede semple

t. semple, .2283 g.

in. o11, .2186 g.

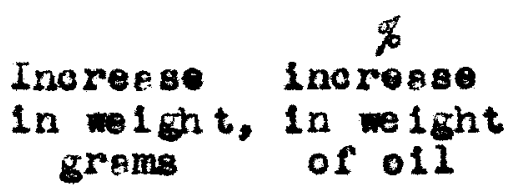

0.0048

0.0140

0.0180

$0.0 \overline{24} 1$

0.0244

0.0247

0.0250<smiles>[3H]</smiles>

0.0254

$-$

0.0248

-

0.0243

$$
2 \cdot 2
$$

6.4

8.7

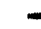

11.0

11.2

11.3

11.4

$-$

11.6

$-$

11.3

$-$

11.1
1 months eging

wt. semple, $1866 \mathrm{~g}$ it. o11, .1788 g.

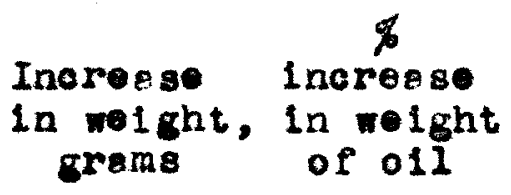

62 
Teble 31

Drying dete for linseed oll ontelning .5 sram leed, Es litherge, .04 grem oobelt end 3.5 grams eerbon b1 ck. (Peroent o11, 95.6)

IIme,

Hours

1
2
3
4
5
6
7
8
10
13
14
21
22
38
62
Freshly rade a $\mathrm{ample}$

H. sample, . $1658 \mathrm{~g}$.

t. o11, . $1584 \mathrm{~B}$.

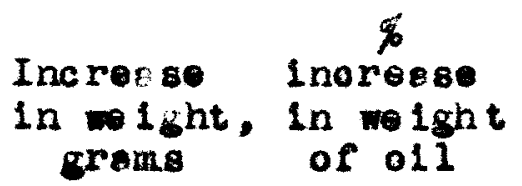

$\begin{array}{cc}0.0070 & 4.4 \\ 0.0135 & 8.5 \\ 0.0163 & 10.3 \\ - & - \\ 0.0197 & 12.4 \\ 0.0186 & 12.4 \\ 0.0198 & 12.5 \\ - & - \\ - & - \\ 0.0197 & 12.4 \\ - & - \\ - & - \\ - & - \\ 0.0185 & 11.7\end{array}$

\section{1 months ering}

t. s mple,.1732 t. ofl, .1658 $\mathrm{g}$.

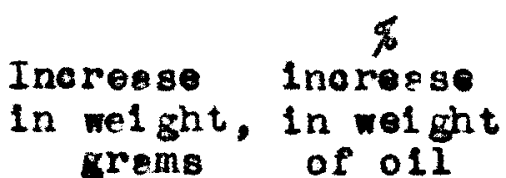
0.0001
0.0050
0.0131
0.0178
0.0186
0.0
3.0
7.8
10.7
11.8
$0.0 \overline{206} \quad 12.4$
$0.0 \overline{209} \quad 12.6$
$0.0209 \quad 12.6$
$0.0208 \quad 12.6$
$0.0197 \quad 11.9$ 


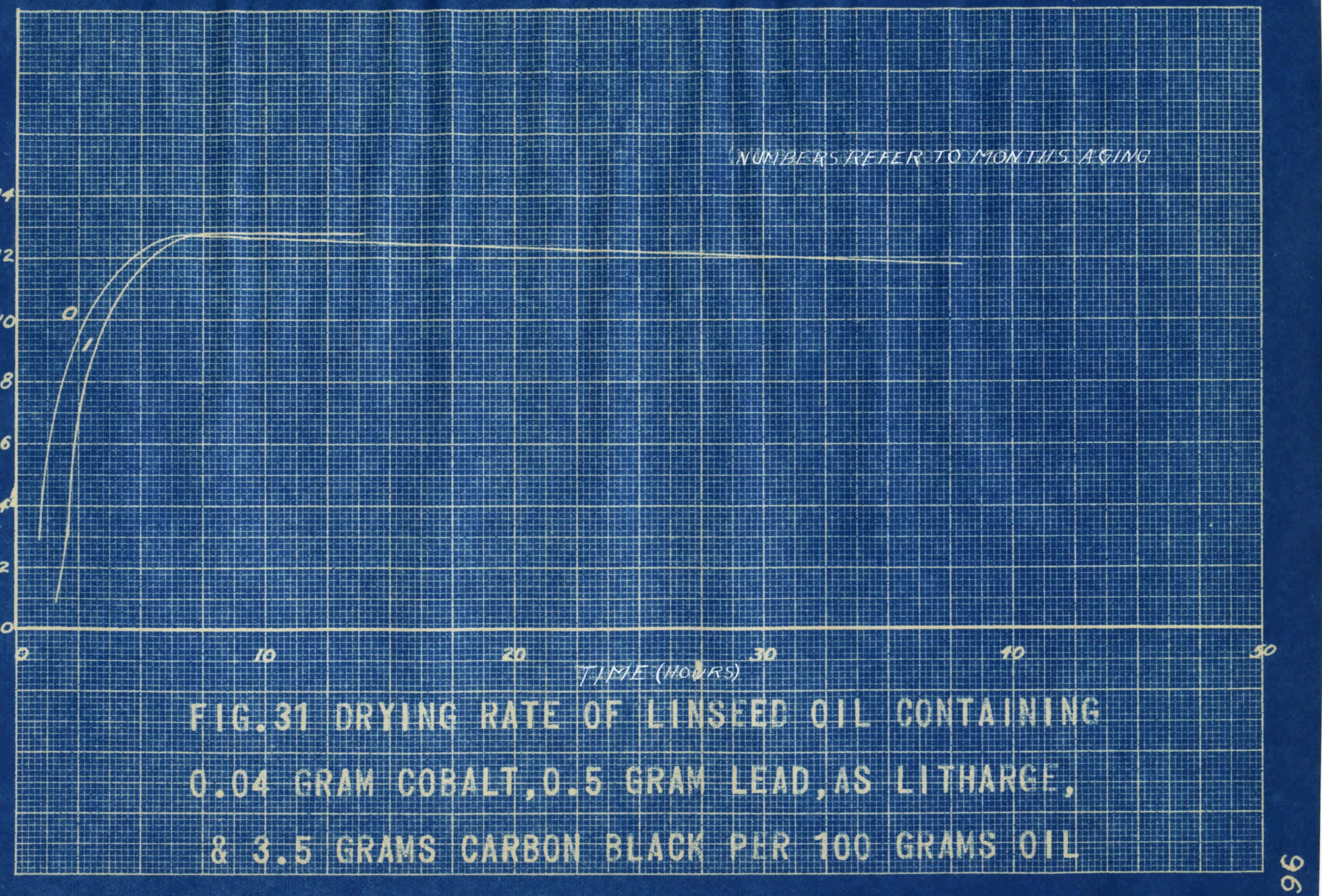


SUMW KY 
Semples of Flgures 24 and 25 treeted with cluminum stospte snd cinc fuodex respetively, and having oobelt s arier, exhiblted very definite decrese in the los of drylng power os compered to Figure 10 untrested plgment. Plgment of semples shown in flgures 27 nd 28 were tres ted for remove 1 of cervon monoxlde and exbon dioxide respectively. Doth indionted decrese in loss of arying ebility over the untres ted plgment (PIgure 10) with tre tment for removel of cerbon aloxide epperently of vins - llghtly better result then trestment for removel of or rbon monox 1de.

Litherge wes us to treat carbon bleck for the same reason thet luminum tearete nd zini Nuodex mere used-to form Ilm round the plgment perticles for keeping arler from belag asorbed re glomerates from forming. The 11 therge treetment gove definite improvement to lose of drying power.

Part of the lost drying power was regelned by regrinding certeln ged emples indiceting thet oome of increan in drying time was os used by entreppment of the drier in aglonere tes (5). 
BIBLI OGRA PHY 
(1) Dennett, hester Thesls, Ef leot of Cinbon Plgments on Lrylng Linseed 011, Speed Sclentific School (1938)

(3) Binney and Smith Compeny, New York, N.Y., bulletin Number 80 , (unpublished, no do te)

(5) Jones and th, 011 and Colour Tredes Jour., 91,341 and $1280(1937)$

(6) Long and macerter, Ind ond Eng. Chen., 23. $786(1931)$

(4) Montreal Production Club, Am. Peint Jour. Convention Delly, Tues., Not. 26 (1937)

(2) Khodes ond Goldsmith, Ind. Eng. Chem., 18, $566(1926)$ 


\section{ACNOF LEDOMBNT}

Tho author wiblos to oxpress his eppreatation to the Leulorille $\mathrm{ALI}$. Fast, and Foniuh club, whose coholarehip mad thle investigaties poesible, and to the Deves and Regnolds Congny. the Bimoy and 8ntth Company. and the Kentudy coler and chomiond company for furathing martials. 